\title{
On the characters of the Sylow $p$-subgroups of untwisted Chevalley groups $Y_{n}\left(p^{a}\right)$
}

\author{
Frank Himstedt, Tung Le and Kay Magaard
}

\begin{abstract}
Let $U Y_{n}(q)$ be a Sylow $p$-subgroup of an untwisted Chevalley group $Y_{n}(q)$ of rank $n$ defined over $\mathbb{F}_{q}$ where $q$ is a power of a prime $p$. We partition the set $\operatorname{Irr}\left(U Y_{n}(q)\right)$ of irreducible characters of $U Y_{n}(q)$ into families indexed by antichains of positive roots of the root system of type $Y_{n}$. We focus our attention on the families of characters of $U Y_{n}(q)$ which are indexed by antichains of length 1. Then for each positive root $\alpha$ we establish a one-to-one correspondence between the minimal degree members of the family indexed by $\alpha$ and the linear characters of a certain subquotient $\bar{T}_{\alpha}$ of $U Y_{n}(q)$. For $Y_{n}=A_{n}$ our single root character construction recovers, among other things, the elementary supercharacters of these groups. Most importantly, though, this paper lays the groundwork for our classification of the elements of $\operatorname{Irr}\left(U E_{i}(q)\right), 6 \leqslant i \leqslant 8$, and $\operatorname{Irr}\left(U F_{4}(q)\right)$.
\end{abstract}

\section{Introduction}

Let $p$ be a prime, $q=p^{a}$ and $Y_{n}(q)$ be a finite quasisimple group of untwisted rank $n$ defined over the field $\mathbb{F}_{q}$. By $U Y_{n}(q)$ we denote a Sylow $p$-subgroup of $Y_{n}(q)$ and by $\operatorname{Irr}(X)$ the set of ordinary irreducible characters of the group $X$.

This paper lays the groundwork for our study of $\operatorname{Irr}\left(U E_{i}(q)\right)$, where $6 \leqslant i \leqslant 8$, and $\operatorname{Irr}\left(U F_{4}(q)\right)$. Our approach is to construct the characters explicitly using as primary parameters the underlying root system and the field. Our focus here is on the families of characters which we parameterize by a single root. For the classical groups, these families can be described recursively via character correspondences which can be achieved using Lemma 2.1. Establishing similar character correspondences for families parameterized by more than one root requires iterated applications of Lemma 2.1. Given the length of the current paper, we treat the recursive method in a sequel.

The solution of the dual problem, the determination of the conjugacy classes of $U Y_{n}(q)$, has been achieved for rank up to 8, but not all of $E_{8}$, in [13-15] by Goodwin, Mosch and Röhrle. Combining these results with the results of this paper and its planned sequel paves the way for the construction of the generic character tables of these groups. For the groups $U D_{4}(q)$ this is presently being carried out by Goodwin, Le and Magaard; see [11].

One motivation for constructing the generic character tables of $U Y_{n}(q)$ is to aid in the construction of the cross characteristic representations and in the determination of the decomposition numbers of the exceptional groups of Lie type. Following Okuyama and Waki $[31,37]$ and Himstedt, Huang and Noeske $[\mathbf{1 9}, \mathbf{2 0}, \mathbf{2 2}]$, we see that characters of parabolic subgroups are a useful tool in the computation of the decomposition numbers of finite groups of Lie type in the cross characteristic case.

A second motivation is to explain exactly why the primes 3 and 5 are bad for the exceptional groups of Lie type from the point of view of the representation theory of $U Y_{n}(q)$. A partial

Received 31 July 2015; revised 7 June 2016.

2010 Mathematics Subject Classification 20C15, 20C33 (primary).

The second and third authors thank the LMS for supporting the second author's visit to Birmingham through grant number 51406 . 
explanation is supplied in Le and Magaard [28] where families of characters are exhibited whose degree is not a power of $q=3^{a}$ or $q=5^{a}$. This generalizes a construction for $U D_{4}(q)$ in $[\mathbf{2 1}]$, where it is shown that there exists exactly one family of characters whose behavior at the prime 2 is different than for odd primes. At present we do not know how many families of $U E_{i}(q)$ characters behave differently at bad primes than at others.

A third motivation is the conjecture of Higman [18] from 1960 that the number of elements of $\operatorname{Irr}\left(U A_{r}\right)$ is a polynomial in $\mathbb{Z}[q]$ and the generalization to other classical groups. This problem has led to the development of supercharacter theories. By grouping conjugacy classes into socalled superclasses and characters into supercharacters these theories allow one to construct supercharacter tables which may be viewed as summarized versions of ordinary character tables. These theories were introduced by Diaconis and Isaacs [6] for algebra groups such as $U A_{n}(q)$. Subsequently, André and Neto [2] developed supercharacter theories for $U B_{n}(q)$, $U C_{n}(q)$ and $U D_{n}(q)$. A common key feature in these theories is that the supercharacters are constructed as tensor products of elementary characters. An open problem in this area is the question of how to split non-elementary supercharacters into ordinary irreducible characters. In his thesis Le [27] shows that the splitting of supercharacters into irreducibles is governed by certain pattern subgroups of $U Y_{n}$ and thus it would suffice to know that Higman's conjecture holds for pattern subgroups. While Higman's conjecture has been verified for $n \leqslant 13$ by VeraLópez and Arregi [36], it was shown by Halasi in [16] and Halasi and Pálfy [17] that it does not hold for pattern groups in general. Pak and Soffer's recent preprint [32] gives strong evidence for why Higman's conjecture may fail for $n \geqslant 59$ while also verifying that the conjecture holds for $n \leqslant 16$.

Our approach to classifying the irreducible characters of $U Y_{n}(q)$ is based on an analysis of the supports of the centers of the characters and character correspondences. We proceed as follows. Let $\Phi$ be a root system of type $Y_{n}$ and let $\Phi^{+}$denote the set of positive roots with respect to some choice of simple roots. The group $U Y_{n}$ is generated by the root subgroups $X_{\alpha}$ where $\alpha \in \Phi^{+}$. For $\chi \in \operatorname{Irr}\left(U Y_{n}\right)$ we define a set $\operatorname{rs}(\chi)$ (see Definition 4) consisting of those elements $\beta \in \Phi^{+}$whose root subgroup $X_{\beta}$ lies in $Z(\chi)$ but not in $\operatorname{Ker}(\chi)$. Subsets of $\Phi^{+}$which arise in this way are called representable. We show that the number $N(\Phi)$ of representable sets in $\Phi^{+}$is a sum of (generalized) Narayana numbers and that $N(\Phi)$ is equal to the number of antichains in the poset of positive roots of $\Phi$ as well as the number of clusters in a cluster algebra of type $\Phi$; see Proposition 5.9.

We say that a character $\chi$ is a single root character if its representable set is non-empty and as small as possible, that is, $|\operatorname{rs}(\chi)|=1$. Each family of single root characters contains a collection of characters of minimal degree which we call midafi characters, and each collection contains a special element that we call a standard midafi. If the root system is of type $A_{n}$ our standard midafi characters are called elementary characters by André [1]; see also [6]. For the root systems of types $B_{n}, C_{n}$ and $D_{n}$ our midafi characters differ from those defined in André and Neto [2]. To see this we note that all of our standard midafi characters are irreducible, whereas not all the elementary characters defined by André and Neto are.

For $\alpha \in \Phi^{+}$we define

$$
\operatorname{Irr}\left(U Y_{n}(q)\right)_{\alpha}:=\left\{\chi \in \operatorname{Irr}\left(U Y_{n}(q)\right) \mid \operatorname{rs}(\chi)=\{\alpha\}\right\},
$$

the set of single root characters lying over $\alpha$, and the set

$$
\operatorname{Irr}^{\text {mida }}\left(U Y_{i}\right)_{\alpha}:=\left\{\mu \in \operatorname{Irr}\left(U Y_{n}(q)\right)_{\alpha} \mid \mu \text { is midafi }\right\} .
$$

The observation that $X_{\beta}$ must act faithfully on any module affording $\chi$ for all $\beta \in \Phi^{+}$ such that $\alpha-\beta \in \Phi^{+}$leads to the definition of the hook $h(\alpha)$ of $\alpha$; see Definition 9. For $\chi \in \operatorname{Irr}\left(U Y_{n}(q)\right)_{\alpha}$ let $k(\alpha):=\left\{\kappa \in \Phi^{+} \mid X_{\kappa} \subseteq \operatorname{Ker}(\chi)\right\}$. We show that, for fixed $\alpha$, the set $k(\alpha) \subseteq \Phi^{+}$does not depend on $\chi$, that $h(\alpha) \cap k(\alpha)=\emptyset$ and that in general $\beta+\gamma \in h(\alpha) \cup k(\alpha)$ 
for all $\beta, \gamma \in h(\alpha)$. Also we will see that typically $H_{\alpha}:=\left\langle X_{\gamma} \mid \gamma \in h(\alpha)\right\rangle$ acts as special group on any module affording $\chi$. As a result we obtain that $\chi(1)=c q^{d}$ with $d=(|h(\alpha)|-1) / 2$. When $c=1, \chi$ is a midafi.

More generally, using our reduction lemma (Lemma 2.1), we can interpret $c$ as the degree of an irreducible character of a suitable quotient $\bar{T}_{\alpha}$ of a certain subgroup $S_{\alpha}<U Y_{n}(q)$. Our main theorems can now be stated under mild hypotheses on the prime $p$ (see Hypothesis 1).

TheOREM 1.1. Let $\Phi_{n}$ be an irreducible root system of type $A_{n}, B_{n}, n \geqslant 2, C_{n}, n \geqslant 3$, or $D_{n}, n \geqslant 4$, and $\mathbb{F}_{q}$ a finite field of characteristic $p$ such that Hypothesis 1 holds. For every positive root $\alpha \in \Phi_{n}^{+}$the map

$$
\Psi: \operatorname{Irr}\left(\bar{T}_{\alpha}\right) \times \operatorname{Irr}\left(X_{\alpha}\right)^{*} \rightarrow \operatorname{Irr}\left(U Y_{n}\right)_{\alpha}, \quad(\mu, \lambda) \mapsto\left(\operatorname{Infl}_{\bar{T}_{\alpha}}^{S_{\alpha}} \mu \cdot \operatorname{Infl}_{X_{\alpha}}^{S_{\alpha}} \lambda\right)^{U Y_{n}}
$$

is a one-to-one correspondence.

Theorem 1.2. Let $i \in\{2,4,6,7,8\}$ and let $\Phi_{i}$ be a root system of type $G_{2}, F_{4}$ or $E_{i}$ respectively and $\mathbb{F}_{q}$ a finite field of characteristic $p$ such that Hypothesis 1 holds. For every positive root $\alpha \in \Phi_{i}^{+}$the map

$$
\Psi: \operatorname{Irr}{ }^{\operatorname{lin}}\left(\bar{T}_{\alpha}\right) \times \operatorname{Irr}\left(X_{\alpha}\right)^{*} \rightarrow \operatorname{Irr}^{\text {mida }}\left(U Y_{i}\right)_{\alpha}, \quad(\mu, \lambda) \mapsto\left(\operatorname{Infl}_{\bar{T}_{\alpha}}^{S_{\alpha}} \mu \cdot \operatorname{Infl}_{X_{\alpha}}^{S_{\alpha}} \lambda\right)^{U Y_{i}}
$$

is a one-to-one correspondence.

For $\Phi$ classical we provide information on the structure of $\bar{T}_{\alpha}$ for every $\alpha \in \Phi^{+}$, whereas for $\Phi$ exceptional we determine for every $\alpha \in \Phi_{i}^{+}$the number of midafis for $\alpha$ and their degrees.

To explain why our results differ for classical and exceptional groups we need to address the issue of how $\bar{T}_{\alpha}$ is constructed. We partition the set $h(\alpha) \backslash\{\alpha\}$ into two subsets of equal size $a(\alpha)$ and $\ell(\alpha)$ which we call the arm and leg respectively of $h(\alpha)$. Then we define the source $s(\alpha):=\Phi^{+} \backslash a(\alpha)$. A priori we have $2^{|a(\alpha)|}$ choices for the sets $a(\alpha)$ and $\ell(\alpha)$. However, if our choice for the arm and leg satisfies the following conditions:

(1) $s(\alpha)$ is closed under addition of roots; and

(2) $\lambda+\sigma \in \ell(\alpha) \cup k(\alpha)$ for all $\lambda \in \ell(\alpha)$ and all $\sigma \in s(\alpha)$ such that $\lambda+\sigma \in \Phi^{+}$;

then we can achieve the hypotheses of Lemma 2.1 to establish the correspondence in Theorem 1.1. In this case the group $S_{\alpha}:=\left\langle X_{\sigma} \mid \sigma \in s(\alpha)\right\rangle$ contains the normal subgroup $K_{\alpha}:=\left\langle X_{\lambda} \mid \lambda \in k(\alpha)\right\rangle$ such that $\bar{S}_{\alpha}$, the image of $S_{\alpha}$ in $U Y_{n}(q) / K_{\alpha}$, contains a normal subgroup $\bar{L}_{\alpha}:=\left\langle\bar{X}_{\lambda} \mid \lambda \in \ell(\alpha)\right\rangle$ such that $\bar{S}_{\alpha} / \bar{L}_{\alpha}=\bar{T}_{\alpha} \times \bar{X}_{\alpha}$.

Conditions (1) and (2) can always be achieved when $\Phi$ is classical or of type $G_{2}$. When $\Phi$ is exceptional, condition (1) can always be achieved. However, when $\Phi$ is of type $E_{8}$, condition (2) cannot be achieved for 46 of the 120 roots of $\Phi^{+}$. The numbers for types $E_{7}, E_{6}$ and $F_{4}$ are 11 out of 63,2 out of 36 , and 2 out 24, respectively. Nevertheless we have the following theorem.

Theorem 1.3. Let $i \in\{2,4,6,7,8\}$ and let $\Phi_{i}$ be a root system of type $G_{2}, F_{4}$ or $E_{i}$ respectively and $\mathbb{F}_{q}$ a finite field of characteristic $p$ such that Hypothesis 1 holds. For every positive root $\alpha \in \Phi_{i}^{+}$for which conditions (1) and (2) above can be achieved the map

$$
\Psi: \operatorname{Irr}\left(\bar{T}_{\alpha}\right) \times \operatorname{Irr}\left(X_{\alpha}\right)^{*} \rightarrow \operatorname{Irr}\left(U Y_{i}\right)_{\alpha}, \quad(\mu, \lambda) \mapsto\left(\operatorname{Inf}_{\bar{T}_{\alpha}}^{S_{\alpha}} \mu \cdot \operatorname{Infl}_{X_{\alpha}}^{S_{\alpha}} \lambda\right)^{U Y_{i}}
$$

is a one-to-one correspondence.

We remark that if $p>3$ then Hypothesis 1 is satisfied for the groups $U G_{2}(q)$ and in this case every irreducible character of $U G_{2}(q)$ has degree $1, q$ or $q^{2}$ and all irreducible characters 
of degree greater than 1 are midafis. We remark further that the number of possible choices for $a(\alpha)$ so that (1) and (2) above are satisfied is $(|h(\alpha)|-1) / 2$ in type $A_{n}$ and much smaller in all other cases.

In the case where no choice of $a(\alpha)$ achieves condition (2) we pick from those choices which satisfy condition (1) the one that minimizes the index of $\bar{L}_{\alpha}$ in its normal closure in $\bar{S}_{\alpha}$. This amounts to minimizing the size of

$$
\bar{\ell}(\alpha):=\left\{\tau \in s(\alpha) \mid \text { there exist } \lambda \in \ell(\alpha) \text { and } \sigma_{i} \in s(\alpha) \text { such that } \tau=\lambda+\sum_{i} \sigma_{i}\right\} .
$$

Let $\bar{L}_{\alpha}:=\left\langle\bar{X}_{\mu} \mid \mu \in \bar{\ell}(\alpha)\right\rangle$. Then $\bar{L}_{\alpha}$ is normal in $\bar{S}_{\alpha}$, and finally we can define the group $\bar{T}_{\alpha}$ in the statement of Theorem 1.2 via $\bar{S}_{\alpha} / \overline{\tilde{L}}_{\alpha} \cong \bar{T}_{\alpha} \times \bar{X}_{\alpha}$.

With the machinery set up in this paper we are able to give full descriptions of the character correspondences for the exceptional groups. It should be noted that if $X_{\alpha}$ projects faithfully into a classical quotient of $U Y_{n}(q)$, then condition (2) can always be achieved. Of the 120 positive roots of $E_{8}$ exactly 53 have the property that $X_{\alpha}$ projects faithfully into a classical quotient. In 46 of the 67 remaining cases condition (2) cannot be achieved. (For $F_{4}, E_{6}$ and $E_{7}$ the numbers are 2 out of 10,2 out of 7 , and 11 out of 23, respectively.) Compounding this is the fact that in those cases where condition (2) cannot be achieved, the descriptions of the single root characters involve up to possibly 15 (generally as many as there are subhooks listed in Table A.2) recursive applications of our reduction lemma and are thus beyond the scope of this article.

An algorithmic treatment of the single root characters will appear in forthcoming articles. The case $\Phi$ of type $F_{4}$ is considered by Goodwin, Le, Magaard and Paolini in [12], where we see that our machinery also generalizes to the case of multiple root characters which we encounter in root systems of exceptional Lie type.

The paper is organized as follows. In $\S 2$ we fix notation and prove Lemma 2.1 which is fundamental to our construction of characters. In $\S 3$ we define closed patterns (additively closed subsets of roots) and the corresponding pattern subgroups, and establish some of their basic properties. This is used in $\S \S 4$ and 5 to define the key terms of this paper, such as hooks, root kernels and representable sets, and to establish their basic properties. We show that the number of representable sets is equal to the number of antichains in the poset of positive roots. In $\S 6$ we study single root characters to lay the foundations for establishing our correspondences. For the classical root systems our main theorem is established in $\S 7$ and for the exceptional root systems in $\S 8$.

\section{Notation and a reduction lemma}

In this section we fix our notation and prove the key lemma which is needed to establish the character correspondences in $\S \S 7$ and 8 .

\subsection{Character-theoretic setting}

For any finite group $U$ let $\operatorname{Irr}(U)$ be the set of complex irreducible characters of $U$ and $\operatorname{Irr}^{\operatorname{lin}}(U):=\{\chi \in \operatorname{Irr}(U) \mid \chi(1)=1\}$ the set of linear characters. Let $(\cdot, \cdot)_{U}$ or $(\cdot, \cdot)$ be the usual scalar product on the space of $\mathbb{C}$-valued class functions of $U$. We write $\mathbf{1}_{U}$ or $\mathbf{1}$ for the trivial character of $U$ and set $\operatorname{Irr}(U)^{*}:=\operatorname{Irr}(U) \backslash\left\{\mathbf{1}_{U}\right\}$. Suppose that $N$ is a normal subgroup of $U$ and that $H$ is a (not necessarily normal) subgroup of $U$. If $\chi$ is a character of $U, \lambda$ is a character of $H$ and $\psi$ is a character of the factor group $U / N$, we write $\lambda^{U}$ for the character of $U$ induced by $\lambda,\left.\chi\right|_{H}$ for the restriction of $\chi$ to $H$ and $\operatorname{Infl}_{U / N}^{U} \psi$ for the inflation 
of $\psi$ to $U$. For $\lambda \in \operatorname{Irr}(H)$ we set

$$
\operatorname{Irr}(U, \lambda):=\left\{\chi \in \operatorname{Irr}(U) \mid\left(\chi, \lambda^{U}\right)>0\right\}
$$

and

$$
\operatorname{Irr}(U / N, \lambda):=\{\chi \in \operatorname{Irr}(U, \lambda) \mid N \subseteq \operatorname{Ker}(\chi)\},
$$

where $\operatorname{Ker}(\chi)$ denotes the kernel of $\chi$. The following lemma provides a character correspondence between finite groups and certain subgroups.

Lemma 2.1. Let $H$ be a subgroup of a finite group $U$ and $X$ a set of representatives for $U / H$. Furthermore, let $Y, Z$ be subgroups of $H$ and $\lambda \in \operatorname{Irr}(Z)$ such that:

(a) $Z \subseteq Z(U)$,

(b) $Y \unlhd H$,

(c) $Z \cap Y=\{1\}$,

(d) $Z Y \unlhd U$,

(e) for the extension $\tilde{\lambda} \in \operatorname{Irr}(Z Y)=\operatorname{Irr}(Z \times Y)$ of $\lambda$ with $Y \subseteq \operatorname{Ker}(\tilde{\lambda})$ we have ${ }^{x} \tilde{\lambda} \neq \tilde{\lambda}$ for all $x \in X \backslash H$.

Then the map $\Phi: \operatorname{Irr}(H / Y, \lambda) \rightarrow \operatorname{Irr}(U, \lambda) \cap \operatorname{Irr}\left(U, \mathbf{1}_{Y}\right), \chi \mapsto \chi^{U}$ is bijective. If additionally

(f) $|X|=|Y|$

holds, then $\operatorname{Irr}(U, \lambda) \cap \operatorname{Irr}\left(U, \mathbf{1}_{Y}\right)=\operatorname{Irr}(U, \lambda)$.

Proof. Suppose that (a)-(e) are true. By (c)-(e) the character $\tilde{\lambda}$ is an irreducible character of the normal subgroup $Z Y$ of $U$ so that we can apply Clifford theory. For all $h \in H, y \in Y$, $z \in Z$ we have ${ }^{h} \tilde{\lambda}(z y)=\tilde{\lambda}\left(z^{h} y^{h}\right)=\tilde{\lambda}(z y)$ by (a) and (b). So $H$ is contained in the inertia subgroup $I_{U}(\tilde{\lambda})$, and from (e) we get $H=I_{U}(\tilde{\lambda})$. By Clifford theory [25, Theorem (6.11)] the map

$$
\Phi: \operatorname{Irr}(H, \tilde{\lambda}) \rightarrow \operatorname{Irr}(U, \tilde{\lambda}), \chi \mapsto \chi^{U}
$$

is a bijection. Since $\operatorname{Irr}(H, \tilde{\lambda})=\operatorname{Irr}(H / Y, \lambda)$ and $\operatorname{Irr}(U, \tilde{\lambda})=\operatorname{Irr}(U, \lambda) \cap \operatorname{Irr}\left(U, \mathbf{1}_{Y}\right)$ the first claim follows.

Assume additionally that (f) holds. By (a), (e) and (f) the character $\lambda$ has at least $|X|=|Y|$ distinct extensions to $Z Y=Z \times Y$. It follows that $\lambda$ has exactly $|Y|$ distinct extensions to $Z Y=Z \times Y$ and that these are permuted transitively by the conjugation action of $U$. One of these extensions is $\tilde{\lambda}$. Thus each $\mu \in \operatorname{Irr}(Z Y)$ with $\left.\mu\right|_{Z}=\lambda$ is conjugate in $U$ to $\tilde{\lambda}$. Hence

$$
\operatorname{Irr}(U, \lambda) \subseteq \operatorname{Irr}(U, \tilde{\lambda})=\operatorname{Irr}(U, \lambda) \cap \operatorname{Irr}\left(U, \mathbf{1}_{Y}\right) \subseteq \operatorname{Irr}(U, \lambda) .
$$

\subsection{Lie-theoretic setting}

We fix a power $q=p^{a}$ of a prime $p$ and write $\mathbb{F}_{q}$ for a field with $q$ elements. Let $Y_{n}(q)$ be an untwisted Chevalley group defined over $\mathbb{F}_{q}$, constructed from a simple Lie algebra with the irreducible root system $\Phi$ of Dynkin type $Y$ and rank $n$ as described in $[4, \S 4.4]$. So $Y_{n}(q)$ is generated by elements $x_{\alpha}(t)$ for $\alpha \in \Phi$ and $t \in \mathbb{F}_{q}$. Let $X_{\alpha}:=\left\langle x_{\alpha}(t) \mid t \in \mathbb{F}_{q}\right\rangle$ be the root subgroup corresponding to a root $\alpha \in \Phi$.

We fix a set $\Delta=\left\{\delta_{1}, \ldots, \delta_{n}\right\}$ of simple roots and write $\Phi^{+}$for the corresponding set of positive roots. So each $\alpha \in \Phi^{+}$can be written as $\alpha=\sum_{i=1}^{n} m_{i} \delta_{i}$ where the coefficients $m_{i} \geqslant 0$ are integers. We write ht $(\alpha):=\sum_{i=1}^{n} m_{i}$ for the height of $\alpha$.

Let $U Y_{n}(q)$ or $U Y_{n}$ be the subgroup generated by $\left\{x_{\alpha}(t) \mid \alpha \in \Phi^{+}, t \in \mathbb{F}_{q}\right\}$. So $U Y_{n}$ is a maximal unipotent subgroup and a Sylow $p$-subgroup of $Y_{n}(q)$. For example, it is well known that $U A_{5}(q)$ is isomorphic to the subgroup of $\mathrm{SL}_{6}(q)$ consisting of all upper unitriangular 
matrices. Let $\leqslant$ be a total ordering on $\Phi^{+}$. Then each element $u \in U Y_{n}$ can be written uniquely as

$$
u=\prod_{\alpha \in \Phi^{+}} x_{\alpha}\left(t_{\alpha}\right)
$$

where the product is taken over all positive roots in increasing order. The multiplication of the elements of $U Y_{n}$ is determined by commutator relations after fixing the signs of certain structure constants corresponding to the so-called extraspecial pairs of roots; see [4, $\S \S 4.2$ and 5.2] for details.

We say that a non-empty subset $\Psi \subseteq \Phi$ is a root subsystem if $\sigma_{\alpha}(\Psi)=\Psi$ for all reflections $\sigma_{\alpha}$ corresponding to roots $\alpha \in \Psi$. Let $\Psi$ be a root subsystem of $\Phi$ of Dynkin type $Y^{\prime}$ and rank $n^{\prime}$ and let $\mathbb{Z} \Psi$ be the $\mathbb{Z}$-span of $\Psi$. We define $\Psi^{+}:=\Psi \cap \Phi^{+}$and $U_{\Psi}:=\prod_{\alpha \in \Psi^{+}} X_{\alpha}$ where the product is taken over all $\alpha \in \Psi^{+}$in increasing order. If $\mathbb{Z} \Psi \cap \Phi=\Psi$ then the commutator relations and the properties of the structure constants in [4, pp. 58-59] imply that $U_{\Psi}$ is a subgroup of $U Y_{n}$ isomorphic to $U Y_{n^{\prime}}^{\prime}$.

Recall that we can define a partial order $\preceq$ on $\Phi^{+}$as follows (see $[\mathbf{2 3}, 10.1]$ ). For roots $\alpha, \beta \in \Phi^{+}$we write $\alpha \prec \beta$ if $\beta-\alpha$ is a non-zero sum of positive roots and we write $\alpha \preceq \beta$ if $\alpha \prec \beta$ or $\alpha=\beta$. The following lemma is a special case of [35, Lemma 3.2].

Lemma 2.2. For all $\alpha, \beta \in \Phi^{+}$with $\alpha \prec \beta$ there are roots $\gamma_{i} \in \Phi^{+}$such that $\alpha=\gamma_{0} \prec \gamma_{1} \prec$ $\gamma_{2} \prec \ldots \prec \gamma_{s-1} \prec \gamma_{s}=\beta$ and $\gamma_{i}-\gamma_{i-1} \in \Delta$ for all $i=1,2, \ldots, s$.

Proof. The lemma follows from [35, Lemma 3.2].

A chain in $\Phi^{+}$is a subset $C=\left\{\gamma_{1}, \gamma_{2}, \ldots, \gamma_{s}\right\} \subseteq \Phi^{+}$such that $\gamma_{i} \prec \gamma_{i+1}$ for all $1 \leqslant i \leqslant s-1$. We say that $C$ is unrefinable if there is no root $\gamma \in \Phi^{+} \backslash C$ such that $\gamma_{i} \prec \gamma \prec \gamma_{i+1}$ for some $i$. By Lemma 2.2 unrefinable chains have the property that the difference of consecutive elements is a simple root. By [23, Lemma 10.4A] the highest positive root of $\Phi$ is the unique maximal element of the poset $\left(\Phi^{+}, \preceq\right)$.

\section{Pattern subgroups}

In $[\mathbf{2 6}, \S 2]$, Isaacs defines pattern subgroups of the subgroup $U_{m}(q)$ of $\mathrm{GL}_{m}\left(\mathbb{F}_{q}\right)$ consisting of the upper unitriangular matrices. Since $U_{m}(q)$ is isomorphic to $U A_{m-1}(q)$ these pattern subgroups can be identified with subgroups of $U A_{m-1}(q)$ in a natural way. The following definition generalizes the notion of pattern subgroups to other Dynkin types. We assume the setting described in $\S 2$. In particular, $q$ is a power of a prime $p$ and $Y_{n}(q)$ is an untwisted Chevalley group defined over $\mathbb{F}_{q}$ with the irreducible root system $\Phi$. The set of positive roots is denoted by $\Phi^{+}$and $U Y_{n}(q)$ or $U Y_{n}$ is the subgroup of $Y_{n}(q)$ generated by the root subgroups $X_{\alpha}$ for $\alpha \in \Phi^{+}$.

DeFinition 1. Let $S$ be a subset of $\Phi^{+}$.

(a) The set $S$ is called a closed pattern if for all $\operatorname{roots} \alpha, \beta \in S$ we have $\alpha+\beta \in S$ or $\alpha+\beta \notin \Phi^{+}$.

(b) For a closed pattern $S$ let $P(S)$ be the subgroup of $U Y_{n}$ generated by the root subgroups $X_{\alpha}$ for $\alpha \in S$. We call $P(S)$ the pattern subgroup corresponding to $S$.

Note that if we have a total ordering $\leqslant$ on $\Phi^{+}$and a closed pattern $S$ then (2.1), [4, Lemma 3.6.3] and the commutator relations imply that $P(S)=\prod_{\alpha \in S} X_{\alpha}$ where the product is taken over the roots in $S$ in increasing order. Obviously, each root subgroup $X_{\alpha}$ for $\alpha \in \Phi^{+}$ and the trivial subgroups $U Y_{n}=P\left(\Phi^{+}\right)$and $\{1\}=P(\emptyset)$ are pattern subgroups. 
Definition 2. Let $M, N$ be subsets of a closed pattern $S \subseteq \Phi^{+}$.

(a) We say that $M$ is a closed subpattern of $S$ if $M$ is a closed pattern.

(b) The closed pattern generated by $M$ is the intersection of all closed patterns containing $M$.

(c) We say that $M$ normalizes $N$ if for all $\alpha \in M, \beta \in N$ we have $\alpha+\beta \in N$ or $\alpha+\beta \notin \Phi^{+}$. We say that $N$ is normal in $S$ if $S$ normalizes $N$. In this case we write $N \unlhd S$ and call $P(S) / P(N)$ the quotient pattern group of $P(S)$ corresponding to $N$. We say that $N$ is normal if $N$ is normal in $\Phi^{+}$.

(d) The normal closure of $M$ in $S$ is the intersection of all normal closed subpatterns of $S$ containing $M$.

Obviously, the closed pattern generated by $M \subseteq \Phi^{+}$is the smallest closed pattern containing $M$ and the normal closure of $N$ in $S$ is the smallest normal closed subpattern of $S$ containing $M$. Note that the normal closed patterns are exactly the (upper) order ideals of the root poset $\left(\Phi^{+}, \preceq\right)$ in the sense of $[\mathbf{3 5}]$.

REMARK 1. If $N \unlhd S$ then (2.1), [4, Lemma 3.6.3] and the commutator relations imply that $N$ is a closed subpattern of $S$ and $P(N) \unlhd P(S)$. In particular, $P(S) / P(N)$ is a welldefined factor group. Let $\pi: P(S) \rightarrow P(S) / P(N)$ be the canonical projection and $\gamma \in S \backslash N$. Since $\pi$ maps the root subgroup $X_{\gamma}$ injectively into $P(S) / P(N)$ we often identify $X_{\gamma}$ with $\pi\left(X_{\gamma}\right)$.

To avoid degeneracies in the commutator relations we will often assume the following hypothesis.

Hypothesis 1. If the Dynkin diagram of $\Phi$ has a double or triple edge assume that

- $p>2$ if $\Phi$ is of type $B_{m}$ or $C_{m}(m \geqslant 2)$ or $F_{4}$,

- $p>3$ if $\Phi$ is of type $G_{2}$.

Next, we consider the connection between normal closed patterns and normal subgroups. For $x, y \in U Y_{n}$ we set $[x, y]:=x^{-1} y^{-1} x y$. Part (c) of the following lemma is stated in [3] for connected reductive groups; see also [7, 1.12, 1.13]. Since we need it for finite groups we sketch a proof.

Lemma 3.1. Let $\alpha, \beta \in \Phi^{+}$such that $\alpha+\beta \in \Phi^{+}$. We assume that Hypothesis 1 is satisfied and set $\Phi_{\alpha, \beta}^{>0}:=\left\{i \alpha+j \beta \in \Phi^{+} \mid i, j \in \mathbb{Z}_{>0}\right\}$.

(a) For all $s, t \in \mathbb{F}_{q}$ there are constants $c_{i j \alpha \beta} \in \mathbb{F}_{q}$ such that $c_{11 \alpha \beta} \neq 0$ and

$$
x_{\alpha}(s)^{-1} x_{\beta}(t)^{-1} x_{\alpha}(s) x_{\beta}(t)=\prod_{i, j>0} x_{i \alpha+j \beta}\left(c_{i j \alpha \beta} \cdot(-t)^{i} s^{j}\right),
$$

where the product is taken over all $i, j \in \mathbb{Z}_{>0}$, such that $i \alpha+j \beta \in \Phi^{+}$and the terms in the product are ordered from left to right so that $i+j$ is increasing.

(b) The set $\Phi_{\alpha, \beta}^{>0}$ is a closed pattern and a subset of the normal closure of $\{\alpha+\beta\}$ in the closed pattern generated by $\alpha$ and $\beta$.

(c) $\left[3\right.$, Remarque 2.5] $\left[X_{\alpha}, X_{\beta}\right]=\prod_{\gamma \in \Phi_{\alpha, \beta}^{>0}} X_{\gamma}$.

Note that because $\Phi_{\alpha, \beta}^{>0}$ is a closed pattern the product in (c) does not depend on the order of the factors.

Proof. (a) By assumption, $\Phi$ is irreducible. Let $\Phi_{\alpha, \beta}:=\Phi \cap(\mathbb{Z} \alpha+\mathbb{Z} \beta)$. Since $\alpha+\beta \in$ $\Phi$ we know from [4, Lemma 3.6.3] that $\Phi_{\alpha, \beta}$ is a root system of type $A_{2}, B_{2}$ or $G_{2}$. Let 
$-m \alpha+\beta, \ldots, \beta, \ldots, m^{\prime} \alpha+\beta$ be the $\alpha$-string through $\beta$. Then (3.1) holds with $c_{11 \alpha \beta}= \pm(m+1)$ by [4, Corollary 5.2.3 and $\S \S 4.1$ and 4.3]. We have to show that $c_{11 \alpha \beta} \neq 0$.

Suppose that $\Phi$ has type $A_{n}, D_{n}, E_{6}, E_{7}$ or $E_{8}$. Since all roots of $\Phi$ have the same length, $\Phi_{\alpha, \beta}$ has type $A_{2}$ and hence $m=0$; see $[\mathbf{2 3}, 9.3]$. Thus $c_{11 \alpha \beta}= \pm 1$ and $c_{11 \alpha \beta} \neq 0$. Suppose that $\Phi$ has type $B_{n}, C_{n}$ or $F_{4}$. Again the lengths of the roots imply that $\Phi_{\alpha, \beta}$ has type $A_{2}$ or $B_{2}$ and so $m \in\{0,1\}$ by $[\mathbf{2 3}, 9.3]$. Thus $c_{11 \alpha \beta}= \pm 1$ or $c_{11 \alpha \beta}= \pm 2$ and so $c_{11 \alpha \beta} \neq 0$ by Hypothesis 1. Finally, suppose that $\Phi$ has type $G_{2}$. Then $\Phi_{\alpha, \beta}$ has type $A_{2}$ or $G_{2}$ and so $m \in\{0,1,2\}$ by $[\mathbf{2 3}, 9.3]$. Thus $c_{11 \alpha \beta}= \pm 1, \pm 2$ or \pm 3 . Thus $c_{11 \alpha \beta} \neq 0$ because $p>3$.

(b), (c) Obviously, $\Phi_{\alpha, \beta}^{>0}$ is a closed pattern and thus $\prod_{\gamma \in \Phi_{\alpha, \beta}^{>0}} X_{\gamma}$ is a subgroup of $U Y_{n}$. The commutator relations imply that $\left[X_{\alpha}, X_{\beta}\right] \subseteq \prod_{\gamma \in \Phi_{\alpha, \beta}^{>0}} X_{\gamma}$. Since $\Phi_{\alpha, \beta}$ has type $A_{2}, B_{2}$ or $G_{2}$ we see that $\Phi_{\alpha, \beta}^{>0}$ is one of the following sets: $\{\alpha+\beta\},\{\alpha+\beta, 2 \alpha+\beta\},\{\alpha+\beta, \alpha+2 \beta\}$, $\{\alpha+\beta, 2 \alpha+\beta, \alpha+2 \beta\},\{\alpha+\beta, 2 \alpha+\beta, 3 \alpha+\beta, 3 \alpha+2 \beta\},\{\alpha+\beta, \alpha+2 \beta, \alpha+3 \beta, 2 \alpha+3 \beta\}$.

We only treat the most complicated case $\Phi_{\alpha, \beta}^{>0}=\{\alpha+\beta, 2 \alpha+\beta, 3 \alpha+\beta, 3 \alpha+2 \beta\}$. Because $2 \alpha+\beta=\alpha+(\alpha+\beta), 3 \alpha+\beta=\alpha+(2 \alpha+\beta)$ and $3 \alpha+2 \beta=\beta+(3 \alpha+\beta)$ the statement in part (b) follows.

To prove the statement in (c) note that $\left[X_{\alpha}, X_{\beta}\right] \unlhd\left\langle X_{\alpha}, X_{\beta}\right\rangle$; see [24, Hilfssatz III.1.6(b)]. By (a) we have $\left[X_{\alpha}, X_{\beta}\right]=X_{\alpha+\beta} \bmod X_{2 \alpha+\beta} X_{3 \alpha+\beta} X_{3 \alpha+2 \beta}$. Thus, there are $d, d^{\prime}, d^{\prime \prime} \in \mathbb{F}_{q}$ such that $u:=x_{\alpha+\beta}(1) x_{2 \alpha+\beta}(d) x_{3 \alpha+\beta}\left(d^{\prime}\right) x_{3 \alpha+2 \beta}\left(d^{\prime \prime}\right) \in\left[X_{\alpha}, X_{\beta}\right]$. Again it follows from (a) that $\left[X_{\alpha}, u\right]=X_{2 \alpha+\beta} \bmod X_{3 \alpha+\beta} X_{3 \alpha+2 \beta}$ so there are elements $f, f^{\prime} \in \mathbb{F}_{q}$ such that $u^{\prime}:=$ $x_{2 \alpha+\beta}(1) x_{3 \alpha+\beta}(f) x_{3 \alpha+2 \beta}\left(f^{\prime}\right) \in\left[X_{\alpha}, X_{\beta}\right]$. Again from (a) we get that $\left[X_{\alpha}, u^{\prime}\right]=X_{3 \alpha+\beta} \bmod$ $X_{3 \alpha+2 \beta}$ and then $u^{\prime \prime}:=x_{3 \alpha+\beta}(1) x_{3 \alpha+2 \beta}(g) \in\left[X_{\alpha}, X_{\beta}\right]$ for some $g \in \mathbb{F}_{q}$. From (a) we get $\left[X_{\beta}, u^{\prime \prime}\right]=X_{3 \alpha+2 \beta} \subseteq\left[X_{\alpha}, X_{\beta}\right]$. Now we can work backwards and get $X_{3 \alpha+\beta} \subseteq\left[X_{\alpha}, X_{\beta}\right]$ and then $X_{2 \alpha+\beta} \subseteq\left[X_{\alpha}, X_{\beta}\right]$ and, finally, $X_{\alpha+\beta} \subseteq\left[X_{\alpha}, X_{\beta}\right]$, proving (c).

Corollary 3.2. Let $N \subseteq S \subseteq \Phi^{+}$be closed patterns and suppose that Hypothesis 1 holds. Then $N \unlhd S$ if and only if $P(N) \unlhd P(S)$.

Proof. By Remark 1 we already know that $N \unlhd S$ implies $P(N) \unlhd P(S)$ even without the condition on $p$. Now suppose that $P(N) \unlhd P(S)$ and let $\alpha \in S, \beta \in N$ such that $\alpha+\beta \in \Phi^{+}$. By Lemma 3.1(c) we have $X_{\alpha+\beta} \subseteq\left[X_{\alpha}, X_{\beta}\right] \subseteq\left[X_{\alpha}, P(N)\right] \subseteq P(N)$. The remark after Definition 1 and the uniqueness in (2.1) imply that $\alpha+\beta \in N$.

Without Hypothesis 1, the converse in Corollary 3.2 is not true in general. Suppose that $\Phi$ has type $B_{2}$ and that $\{\alpha, \beta\}$ is a set of simple roots. If $p=2$ then $X_{\alpha+\beta} \unlhd U B_{2}(q)$ but the closed pattern $\{\alpha+\beta\}$ is not normal.

The following lemmas are of theoretical and computational use. They show that derived subgroups and centers of quotient pattern groups are compatible with the root structure.

Lemma 3.3. Let $N \subseteq S \subseteq \Phi^{+}$be closed patterns such that $N \unlhd S$ and assume that Hypothesis 1 holds. Then $D:=\left(\{\alpha+\beta \mid \alpha, \beta \in S\} \cap \Phi^{+}\right) \cup N$ is a normal closed subpattern of $S$ and $[P(S) / P(N), P(S) / P(N)]=P(D) / P(N)$. In particular, the derived subgroup of a pattern subgroup is also a pattern subgroup.

Proof. Since $S$ is a closed pattern and $N \subseteq S$ we have $N \subseteq D \subseteq S$. For all $\alpha \in S$, $\beta \in D \subseteq S$ with $\alpha+\beta \in \Phi^{+}$we have $\alpha+\beta \in\{\alpha+\beta \mid \alpha, \beta \in S\} \cap \Phi^{+} \subseteq D$. Hence $D$ is a normal closed subpattern of $S$ and $P(N) \subseteq P(D) \unlhd P(S)$. It follows from Lemma 3.1(c) that $P(D) / P(N) \subseteq[P(S) / P(N), P(S) / P(N)]$. The commutator relations and Lemma 3.1(b) show that $(P(S) / P(N)) /(P(D) / P(N))$ is abelian. Thus $P(D) / P(N)$ is the derived subgroup of $P(S) / P(N)$. 
Lemma 3.4. Let $N \subseteq S \subseteq \Phi^{+}$be closed patterns such that $N \unlhd S$ and assume that Hypothesis 1 holds. Then

$$
Z:=\left\{\alpha \in S \mid \text { for all } \gamma \in S: \alpha+\gamma \notin \Phi^{+} \text {or } \alpha+\gamma \in N\right\} \cup N
$$

is a normal closed subpattern of $S$ and $Z(P(S) / P(N))=P(Z) / P(N)$. In particular, the center $Z(P(S) / P(N))$ is isomorphic to a direct product of root subgroups.

Proof. Suppose that $\alpha \in S$ and $\beta \in Z$ such that $\alpha+\beta \in \Phi^{+}$. By the definition of $Z$ and the normality of $N$ in $S$ we have $\alpha+\beta \in N \subseteq Z$ and hence $Z$ is a normal closed subpattern of $S$ containing $N$. The commutator relations and Lemma 3.1(b) imply that $P(Z) / P(N) \subseteq$ $Z(P(S) / P(N))$.

Now suppose that $u P(N) \in Z(P(S) / P(N)) \backslash\{1\}$. According to (2.1) we write

$$
u=x_{\alpha_{1}}\left(t_{1}\right) \ldots x_{\alpha_{s}}\left(t_{s}\right) x_{\alpha_{s+1}}\left(t_{s+1}\right) \ldots x_{\alpha_{s^{\prime}}}\left(t_{s^{\prime}}\right)
$$

where $\alpha_{i} \in S \backslash N$ and $t_{i} \neq 0$ for $i=1, \ldots, s^{\prime}$ and $m:=\operatorname{ht}\left(\alpha_{1}\right)=\ldots=\operatorname{ht}\left(\alpha_{s}\right)<\operatorname{ht}\left(\alpha_{s+1}\right) \leqslant$ $\operatorname{ht}\left(\alpha_{s+2}\right) \leqslant \ldots \leqslant \operatorname{ht}\left(\alpha_{s^{\prime}}\right)$. We show that $\alpha_{i} \in Z$ for $i=1, \ldots, s^{\prime}$ by downwards induction on $m$.

If $m=\max \left\{\operatorname{ht}(\alpha) \mid \alpha \in \Phi^{+}\right\}$then $s=s^{\prime}=1$ and $\alpha_{1}+\gamma \notin \Phi^{+}$for all $\gamma \in S$ and thus $\alpha_{1} \in Z$. Assume that $m<\max \left\{\operatorname{ht}(\alpha) \mid \alpha \in \Phi^{+}\right\}$. Let $\gamma \in S$ and $M$ be the normal subgroup of $P(S)$ generated by all $X_{\alpha}$ to roots $\alpha \in S$ such that $\alpha \in N$ or ht $(\alpha)>m+\operatorname{ht}(\gamma)$. By Lemma 3.1(a) there exist $\tilde{t}_{1}, \ldots, \tilde{t}_{s} \in \mathbb{F}_{q} \backslash\{0\}, \tilde{u} \in M$ such that

$$
x_{\gamma}(1)^{-1} u x_{\gamma}(1)=x_{\alpha_{1}}\left(t_{1}\right) x_{\alpha_{1}+\gamma}\left(\tilde{t}_{1}\right) \ldots x_{\alpha_{s}}\left(t_{s}\right) x_{\alpha_{s}+\gamma}\left(\tilde{t}_{s}\right) x_{\alpha_{s+1}}\left(t_{s+1}\right) \ldots x_{\alpha_{s^{\prime}}}\left(t_{s^{\prime}}\right) \tilde{u},
$$

where we set $x_{\alpha_{i}+\gamma}\left(\tilde{t}_{i}\right):=1$ if $\alpha_{i}+\gamma \notin \Phi^{+}$. It follows from the uniqueness in (2.1) that $\alpha_{i}+\gamma \notin \Phi^{+}$or $\alpha_{i}+\gamma \in N$ for $i=1, \ldots, s$ and therefore $\alpha_{1}, \ldots, \alpha_{s} \in Z$. By induction we get $\alpha_{s+1}, \ldots, \alpha_{s^{\prime}} \in Z$, completing the proof.

Definition 3. Let $N \subseteq S \subseteq \Phi^{+}$be closed patterns such that $N \unlhd S$. Assume that Hypothesis 1 holds and let $Z$ be the closed subpattern of $S$ defined in Lemma 3.4. We call the set $\operatorname{rz}(P(S) / P(N)):=Z \backslash N$ the root center of $P(S) / P(N)$.

\section{Root kernels and root centers}

We keep the setting from the previous sections. In particular, $q$ is a power of a prime $p$ and $Y_{n}(q)$ is an untwisted Chevalley group defined over $\mathbb{F}_{q}$ with an irreducible root system $\Phi$. In this section we associate with each irreducible character $\chi$ of a pattern subgroup $P(S)$ of $U Y_{n}(q)$ certain sets of roots and pattern subgroups of $P(S)$. We assume throughout this section that Hypothesis 1 holds.

Definition 4. Let $S \subseteq \Phi^{+}$be a closed pattern. For $\chi \in \operatorname{Irr}(P(S))$ we set

$$
\begin{aligned}
& \operatorname{rk}(\chi):=\left\{\alpha \in S \mid X_{\alpha} \subseteq \operatorname{Ker}(\chi)\right\}, \\
& \operatorname{rz}(\chi):=\left\{\alpha \in S \mid X_{\alpha} \subseteq Z(\chi)\right\}, \\
& \operatorname{rs}(\chi):=\operatorname{rz}(\chi) \backslash \operatorname{rk}(\chi),
\end{aligned}
$$

and call $\mathrm{rk}(\chi)$ the root kernel, $\mathrm{rz}(\chi)$ the root center and $\mathrm{rs}(\chi)$ the central root support of $\chi$. Associated with these sets of roots are the following groups:

$$
\begin{aligned}
\operatorname{rKer}(\chi) & :=\left\langle X_{\alpha} \mid \alpha \in \operatorname{rk}(\chi)\right\rangle \subseteq P(S) \\
\mathrm{rZ}(\chi) & :=\left\langle X_{\alpha} \mid \alpha \in \mathrm{rz}(\chi)\right\rangle \subseteq P(S) \\
\mathrm{rS}(\chi) & :=\mathrm{rZ}(\chi) / \mathrm{rKer}(\chi) \subseteq P(S) / \mathrm{rKer}(\chi)
\end{aligned}
$$


The next lemma shows that root kernels and root centers behave in much the same way as the usual kernels and centers of irreducible characters. It also implies that $\operatorname{rS}(\chi)$ is indeed a factor group.

Lemma 4.1. Let $S \subseteq \Phi^{+}$be a closed pattern and let $\chi \in \operatorname{Irr}(P(S))$. Then we have the following:

(a) $\operatorname{rk}(\chi)$ and $\mathrm{rz}(\chi)$ are closed patterns which are normal in $S$;

(b) $\operatorname{rKer}(\chi)$ and $\mathrm{rZ}(\chi)$ are normal subgroups of $P(S)$;

(c) the factor groups $P(S) / \mathrm{rKer}(\chi), P(S) / \mathrm{rZ}(\chi)$ are quotient pattern groups;

(d) $\mathrm{rZ}(\chi) / \operatorname{rKer}(\chi)=Z(P(S) / \operatorname{rKer}(\chi))$.

Proof. (a) Let $\alpha \in S$ and $\beta \in \operatorname{rk}(\chi)$ such that $\alpha+\beta \in \Phi^{+}$. By definition, we have $X_{\beta} \subseteq$ $\operatorname{Ker}(\chi) \unlhd P(S)$. Thus Lemma 3.1(c) implies $X_{\alpha+\beta} \subseteq\left[X_{\alpha}, X_{\beta}\right] \subseteq \operatorname{Ker}(\chi)$ and so $\alpha+\beta \in \operatorname{rk}(\chi)$. It follows that $\operatorname{rk}(\chi)$ is a closed pattern which is normal in $S$. The proof for $\operatorname{rz}(\chi)$ is analogous.

(b), (c) follow from (a) and Remark 1.

(d) By part (a) and Lemma 3.4 there is a closed pattern $Z$ such that $\operatorname{rk}(\chi) \subseteq Z \subseteq S$ and $P(Z) / \mathrm{rKer}(\chi)=Z(P(S) / \mathrm{rKer}(\chi))$. Obviously, $Z \subseteq \mathrm{rz}(\chi)$. Let $\alpha \in S$ and $\beta \in \mathrm{rz}(\chi)$. By Lemma 3.1(c) we have $X_{\alpha+\beta} \subseteq\left[X_{\alpha}, X_{\beta}\right] \subseteq\left[X_{\alpha}, Z(\chi)\right] \subseteq \operatorname{Ker}(\chi)$. Hence $\alpha+\beta \in \operatorname{rk}(\chi) \unlhd S$ and Lemma 3.1(b) implies that $i \alpha+j \beta \in \operatorname{rk}(\chi)$ for all positive integers $i, j$. Hence, $\left[X_{\alpha}, X_{\beta}\right]=1$ modulo $\operatorname{rKer}(\chi)$ by Lemma 3.1(c) and we can conclude $\mathrm{rz}(\chi) \subseteq Z$.

Without Hypothesis 1 the statements in Lemma 4.1 are not always true. Suppose that $\Phi$ has type $B_{2}$ and that $\{\alpha, \beta\}$ is a set of simple roots, where $\alpha$ is short. If $p=q=2$ then $N:=\left\{x_{\alpha}\left(d_{1}\right) x_{\beta}\left(d_{2}\right) x_{\alpha+\beta}\left(d_{3}\right) x_{2 \alpha+\beta}\left(d_{3}\right) \mid d_{1}, d_{2}, d_{3} \in \mathbb{F}_{q}\right\}$ is a normal subgroup of $U B_{2}(2)$ of index 2. Let $\chi \in \operatorname{Irr}\left(U B_{2}(2)\right)$ be the non-trivial linear character with $\operatorname{Ker}(\chi)=N$. Then $\operatorname{rk}(\chi)=\{\alpha, \beta\}$ and this is not a closed pattern.

Lemma 4.2. Let $N \unlhd \Phi^{+}$and $\Sigma=\left\{\alpha_{1}, \ldots, \alpha_{s}\right\}=\operatorname{rz}\left(U Y_{n} / P(N)\right)$.

(a) The closed pattern $N$ is the unique maximal element of the set $\left\{M \unlhd \Phi^{+} \mid M \cap \Sigma=\emptyset\right\}$.

(b) For all irreducible characters $\chi \in \operatorname{Irr}\left(U Y_{n}\right)$ the following are equivalent:

(i) $\operatorname{rs}(\chi)=\Sigma$;

(ii) $\chi$ is a constituent of $\lambda^{U Y_{n}}$ for some linear character $\lambda$ of $P(\Sigma \cup N)$ satisfying $P(N) \subseteq$ $\operatorname{Ker}(\lambda)$ and $\left.\lambda\right|_{X_{\alpha_{i}}} \neq \mathbf{1}_{X_{\alpha_{i}}}$ for $i=1,2, \ldots, s$.

If the conditions (i) and (ii) hold then $N=\operatorname{rk}(\chi)$.

Proof. (b) By definition we have $N \unlhd \Phi^{+}$and $\Sigma \cap N=\emptyset$. We identify $X_{\alpha_{i}}$ with its image in $U Y_{n} / P(N)$, so $Z\left(U Y_{n} / P(N)\right)=X_{\alpha_{1}} \times \ldots \times X_{\alpha_{s}}$.

(i) $\Rightarrow$ (ii) Suppose that $\operatorname{rs}(\chi)=\Sigma$ and let $M$ be a normal closed pattern with $M \cap \Sigma=\emptyset$. If $M \nsubseteq \operatorname{rk}(\chi)$ then we choose $\beta \in M \backslash \operatorname{rk}(\chi)$ such that $\operatorname{ht}(\beta)$ is maximal. Let $\alpha \in \Phi^{+}$. Since $M \unlhd \Phi^{+}$the maximality of ht $(\beta)$ implies that $\alpha+\beta \notin \Phi^{+}$or $\alpha+\beta \in \operatorname{rk}(\chi)$ and therefore $\beta \in \mathrm{rz}(\chi)$ by Lemmas 3.4 and $4.1(\mathrm{~d})$. Thus $\beta \in \mathrm{rz}(\chi) \backslash \operatorname{rk}(\chi)=\operatorname{rs}(\chi)=\Sigma$, a contradiction. Hence $M \subseteq \operatorname{rk}(\chi)$. In particular, we have $N \subseteq \operatorname{rk}(\chi)$ and we can identify $\chi$ with some $\tilde{\chi} \in$ $\operatorname{Irr}\left(U Y_{n} / P(N)\right)$. Because $\operatorname{rs}(\chi)=\Sigma$ we have $X_{\alpha_{i}} \nsubseteq \operatorname{Ker}(\tilde{\chi})$ for all $i$. Thus there is a linear character $\tilde{\lambda} \in \operatorname{Irr}\left(X_{\alpha_{1}} \times \ldots \times X_{\alpha_{s}}\right)$ with $\left.\tilde{\lambda}\right|_{X_{\alpha_{i}}} \neq \mathbf{1}_{X_{\alpha_{i}}}$ for all $i$ such that $\tilde{\chi}$ is a constituent of $\tilde{\lambda}^{U Y_{n} / P(N)}$. Hence $\lambda:=\operatorname{Infl}_{P(\Sigma \cup N) / P(N)}^{P(\Sigma \cup N)} \tilde{\lambda}$ has the properties described in (ii).

(ii) $\Rightarrow$ (i) Let $\chi$ be a constituent of $\lambda^{U Y_{n}}$ for some linear character $\lambda$ of $P(\Sigma \cup N)$ with $P(N) \subseteq \operatorname{Ker}(\lambda)$ and $\left.\lambda\right|_{X_{\alpha_{i}}} \neq \mathbf{1}_{X_{\alpha_{i}}}$ for $i=1,2, \ldots, s$. By construction, we have $N \subseteq \operatorname{rk}(\chi)$ and $\Sigma \cap \operatorname{rk}(\chi)=\emptyset$. Assume that $N \neq \operatorname{rk}(\chi)$. Then there is $\beta \in \operatorname{rk}(\chi) \backslash N$ such that $\operatorname{ht}(\beta)$ is maximal. Let $\alpha \in \Phi^{+}$. Since $\operatorname{rk}(\chi) \unlhd \Phi^{+}$the maximality of ht $(\beta)$ implies that $\alpha+\beta \notin \Phi^{+}$or $\alpha+\beta \in N$ and therefore $\beta \in \operatorname{rz}\left(U Y_{n} / P(N)\right)$ by Lemma 3.4. Hence $\beta \in \Sigma$, a contradiction. Thus $\operatorname{rk}(\chi)=N$. Lemma 4.1(d) implies that $\operatorname{rs}(\chi)=\Sigma$, so that (i) holds. 
(a) Let $\chi$ be an irreducible constituent of $\lambda^{U Y_{n}}$ where $\lambda$ is a linear character of $P(\Sigma \cup N)$ as in (b) (ii). We have already seen in the proof of (i) $\Rightarrow$ (ii) that $N$ is an element of the set $\left\{M \unlhd \Phi^{+} \mid M \cap \Sigma=\emptyset\right\}$ and that each element $M$ of this set is a subset of $\operatorname{rk}(\chi)$. In the proof of (ii) $\Rightarrow$ (i) we showed that $\operatorname{rk}(\chi)=N$. This completes the proof of the lemma.

\section{Representable sets}

We keep the setting from the previous sections. In particular, $q$ is a power of a prime $p$ and $Y_{n}(q)$ is an untwisted Chevalley group defined over $\mathbb{F}_{q}$ with an irreducible root system $\Phi$. In this section we use the central root support $\operatorname{rs}(\chi)$ of characters $\chi$ of a pattern subgroup $P(S)$ of $U Y_{n}(q)$ to obtain a partition of $\operatorname{Irr}(P(S))$ which is well adapted to the Lie-theoretic setting. We assume throughout this section that Hypothesis 1 holds.

Definition 5. Let $\Sigma \subseteq \Phi^{+}$. We say that the set $\Sigma$ is representable if there exists $\chi \in$ $\operatorname{Irr}\left(U Y_{n}\right)$ such that $\operatorname{rs}(\chi)=\Sigma$. In this case we define

$$
\operatorname{Irr}\left(U Y_{n}\right)_{\Sigma}:=\left\{\chi \in \operatorname{Irr}\left(U Y_{n}\right) \mid \operatorname{rs}(\chi)=\Sigma\right\}
$$

Obviously, $\operatorname{Irr}\left(U Y_{n}\right)$ is partitioned by the sets $\operatorname{Irr}\left(U Y_{n}\right)_{\Sigma}$ where $\Sigma$ ranges over the representable subsets of $\Phi^{+}$. The next lemma gives a characterization of the representable sets.

Lemma 5.1. For a subset $\Sigma \subseteq \Phi^{+}$the following are equivalent:

(a) the set $\Sigma$ is representable;

(b) there is a closed pattern $N \unlhd \Phi^{+}$such that $\Sigma=\operatorname{rz}\left(U Y_{n} / P(N)\right)$;

(c) there is a unique closed pattern $N \unlhd \Phi^{+}$such that $\Sigma=\operatorname{rz}\left(U Y_{n} / P(N)\right)$.

Proof. (a) $\Rightarrow$ (b) Suppose that $\Sigma$ is representable and let $\chi \in \operatorname{Irr}\left(U Y_{n}\right)$ such that $\operatorname{rs}(\chi)=\Sigma$. We set $N:=\operatorname{rk}(\chi)$. Then $\Sigma=\operatorname{rz}\left(U Y_{n} / P(N)\right)$ by Lemma 4.1(a), (d) with $S=\Phi^{+}$.

(b) $\Rightarrow$ (c) follows from Lemma 4.2(a).

(c) $\Rightarrow$ (a) Choose a linear character $\lambda \in P(\Sigma \cup N)$ as in Lemma 4.2(b)(ii) and let $\chi \in \operatorname{Irr}\left(U Y_{n}\right)$ be a constituent of $\lambda^{U Y_{n}}$. By Lemma 4.2(b) we have $\operatorname{rs}(\chi)=\Sigma$ and (a) follows.

DeFinition 6. Let $\Sigma \subseteq \Phi^{+}$be representable.

(a) We write $k(\Sigma)$ for the unique normal closed pattern with

$$
\Sigma=\mathrm{rz}\left(U Y_{n} / P(k(\Sigma))\right)
$$

(b) We say that a character $\chi \in \operatorname{Irr}\left(U Y_{n} / P(k(\Sigma))\right)$ is almost faithful with respect to $\Sigma$ if $X_{\alpha} \not \subset \operatorname{Ker}(\chi)$ for all $\alpha \in \Sigma$.

The following remark is an immediate consequence of Lemmas 4.1(d) and 5.1.

REMARK 2. Let $\Sigma \subseteq \Phi^{+}$be representable. Then:

(a) $k(\Sigma)$ is the largest element of the set $\left\{M \unlhd \Phi^{+} \mid M \cap \Sigma=\emptyset\right\}$;

(b) inflation induces a one-to-one correspondence between the set of almost faithful irreducible characters of $U Y_{n} / P(k(\Sigma))$ and $\operatorname{Irr}\left(U Y_{n}\right)_{\Sigma}$;

(c) $\operatorname{Irr}\left(U Y_{n}\right)_{\Sigma}=\left\{\chi \in \operatorname{Irr}\left(U Y_{n}\right) \mid \operatorname{rk}(\chi)=k(\Sigma)\right\}$.

Next, we investigate some elementary properties of $k(\Sigma)$. 
Lemma 5.2. Let $\Sigma \subseteq \Phi^{+}$be representable. For all $\alpha \in \Sigma$ and $\gamma \in \Phi^{+}$we have $\alpha+\gamma \notin \Phi^{+}$ or $\alpha+\gamma \in k(\Sigma)$.

Proof. This follows from the definition of $k(\Sigma)$ and Lemma 3.4.

Definition 7. For $\alpha \in \Phi^{+}$let $n_{0}(\alpha):=\{\alpha\}$ and, for $i \geqslant 1$,

$$
n_{i}(\alpha):=\left\{\beta+\gamma \mid \beta \in n_{i-1}(\alpha), \gamma \in \Phi^{+}\right\} \cap \Phi^{+} .
$$

Finally, we set $n(\alpha):=\bigcup_{i \geqslant 1} n_{i}(\alpha)$.

Note that $\alpha \notin n(\alpha)$ since the union is over all $i \geqslant 1$. In fact, $n(\alpha)$ is the normal closure of $\{\alpha\}$ in $\Phi^{+}$with $\alpha$ removed. Also note that by construction, for all $\beta \in n(\alpha)$ and $\gamma \in \Phi^{+}$we have $\beta+\gamma \notin \Phi^{+}$or $\beta+\gamma \in n(\alpha)$; hence $n(\alpha) \unlhd \Phi^{+}$.

LEMma 5.3. If $\Sigma \subseteq \Phi^{+}$is representable then $\bigcup_{\alpha \in \Sigma} n(\alpha) \subseteq k(\Sigma)$.

Proof. Let $\alpha \in \Sigma$. By Lemma 5.2 we have $n_{1}(\alpha) \subseteq k(\Sigma)$. Suppose that $i>1$. Let $\gamma \in \Phi^{+}$ and $\beta \in n_{i-1}(\alpha)$ such that $\beta+\gamma \in \Phi^{+}$. By induction, $\beta \in k(\Sigma)$. Since $k(\Sigma) \unlhd \Phi^{+}$we get $\beta+\gamma \in k(\Sigma)$ and the claim follows.

In the following we show that for each $\alpha \in \Phi^{+}$the set $\{\alpha\}$ is representable and we obtain a recursive description of $k(\{\alpha\})$.

Definition 8. Let $\alpha \in \Phi^{+}$. We define $k_{0}(\alpha):=n(\alpha)$ and, for $i \geqslant 1$,

$$
k_{i}(\alpha):=\left(\operatorname{rz}\left(U Y_{n} / P\left(k_{i-1}(\alpha)\right)\right) \backslash\{\alpha\}\right) \cup k_{i-1}(\alpha) .
$$

Finally, we set $k(\alpha):=\bigcup_{i \geqslant 0} k_{i}(\alpha)$.

Note that $k_{i}(\alpha) \unlhd \Phi^{+}$for all $i \geqslant 0$. This is clear for $i=0$ since $n(\alpha) \unlhd \Phi^{+}$. Suppose that $i>0$. By induction $k_{i-1}(\alpha) \unlhd \Phi^{+}$. Let $\beta \in k_{i}(\alpha)$ and $\gamma \in \Phi^{+}$such that $\beta+\gamma \in \Phi^{+}$. Thus $\beta \in \operatorname{rz}\left(U Y_{n} / P\left(k_{i-1}(\alpha)\right)\right)$ or $\beta \in k_{i-1}(\alpha)$. In both cases we have $\beta+\gamma \in k_{i-1}(\alpha) \subseteq k_{i}(\alpha)$. Hence $k_{i}(\alpha) \unlhd \Phi^{+}$. It follows that $k(\alpha)$ is well defined and that $k(\alpha) \unlhd \Phi^{+}$.

Lemma 5.4. For all $\alpha \in \Phi^{+}$the set $\{\alpha\}$ is representable and $k(\{\alpha\})=k(\alpha)$.

Proof. We have just seen that $k(\alpha) \unlhd \Phi^{+}$and by definition of $k(\alpha)$ we have $\alpha \notin k(\alpha)$. Since $n_{1}(\alpha) \subseteq n(\alpha) \subseteq k(\alpha)$ we have $\{\alpha\} \subseteq \mathrm{rz}\left(U Y_{n} / P(k(\alpha))\right)$. Suppose that $\beta \in \mathrm{rz}\left(U Y_{n} / P(k(\alpha))\right)$. Then $\beta+\gamma \notin \Phi^{+}$or $\beta+\gamma \in k(\alpha)$ for all positive roots $\gamma$. By the definition of $k(\alpha)$ we have $\beta \in k_{i}(\alpha)$ for some $i$ or $\beta=\alpha$. Because $\operatorname{rz}\left(U Y_{n} / P(k(\alpha))\right) \cap k(\alpha)=\emptyset$ we have $\beta=\alpha$. Hence $\{\alpha\}=\operatorname{rz}\left(U Y_{n} / P(k(\alpha))\right)$, so that condition (b) of Lemma 5.1 is satisfied with $N=k(\alpha)$ and the claim follows.

Next, we define a set $w(\alpha) \subseteq \Phi^{+} \backslash k(\alpha)$. The definition of $w(\alpha)$ requires the concept of hooks.

Definition 9. Let $\alpha \in \Phi^{+}$. We call the set

$$
h(\alpha):=\left\{\gamma \in \Phi^{+} \mid \alpha-\gamma \in \Phi^{+} \cup\{0\}\right\}
$$

the hook corresponding to $\alpha$. A subset $h^{\prime}(\alpha) \subseteq h(\alpha)$ is called a subhook of $h(\alpha)$ if $\alpha \in h^{\prime}(\alpha)$ and $\alpha-\gamma \in h^{\prime}(\alpha) \cup\{0\}$ for all $\gamma \in h^{\prime}(\alpha)$.

The term hooks is motivated by the case where $\Phi$ is of type $A_{n}$; see $[\mathbf{2 1}, \S 3]$. The following definition is in some sense dual to that of $k(\alpha)$. 
Definition 10. Let $\alpha \in \Phi^{+}$. We define $w_{0}(\alpha):=\{\alpha\}$ and, for $i \geqslant 1$,

$$
w_{i}(\alpha):=\bigcup_{\beta \in w_{i-1}(\alpha)} h(\beta)
$$

Finally, we set $w(\alpha):=\bigcup_{i \geqslant 0} w_{i}(\alpha)$.

The set $w(\alpha)$ has the following interpretation in terms of the root poset $\left(\Phi^{+}, \preceq\right)$.

Lemma 5.5. For all $\alpha \in \Phi^{+}$we have $w(\alpha)=\left\{\beta \in \Phi^{+} \mid \beta \preceq \alpha\right\}$.

Proof. $\subseteq$ Let $\beta \in w(\alpha)$. Then there is some $i$ such that $\beta \in w_{i}(\alpha)$. We use induction on $i$ to show that $\beta \preceq \alpha$. If $i=0$ we have $\beta=\alpha \preceq \alpha$ so that we can assume $i>0$. Hence there is a root $\gamma \in w_{i-1}(\alpha)$ such that $\beta \in h(\gamma)$ and by induction we have $\beta \preceq \gamma \preceq \alpha$ and therefore $\beta \preceq \alpha$.

$\supseteq$ Let $\beta \in \Phi^{+}$with $\beta \preceq \alpha$. We can assume that $\beta \neq \alpha$. By Lemma 2.2 there are $\gamma_{i} \in \Phi^{+}$ such that $\alpha=\gamma_{0} \succ \gamma_{1} \succ \gamma_{2} \succ \ldots \succ \gamma_{s-1} \succ \gamma_{s}=\beta$ and $\gamma_{i}-\gamma_{i+1}$ is a simple root for all $i$. In particular, $\gamma_{i+1} \in h\left(\gamma_{i}\right)$ for all $i$. It follows that $\gamma_{i} \in w_{i}(\alpha)$ and hence $\beta \in w_{s}(\alpha) \subseteq w(\alpha)$.

If $\alpha_{h} \in \Phi^{+}$is the highest root then Lemma 5.5 and the remarks after Lemma 2.2 imply that $w\left(\alpha_{h}\right)=\Phi^{+}$.

Lemma 5.6. For each $\alpha \in \Phi^{+}$we have $w(\alpha) \cap k(\alpha)=\emptyset$ and $w(\alpha) \cup k(\alpha)=\Phi^{+}$.

Proof. We show $w_{i}(\alpha) \cap k(\alpha)=\emptyset$ for all $i \geqslant 0$ by induction on $i$. By definition of $n(\alpha)$ and $k(\alpha)$ we have $\alpha \notin k(\alpha)$, so that we can assume $i>0$. Suppose that $\beta \in w_{i}(\alpha) \cap k(\alpha)$. By definition of $w_{i}(\alpha)$ there is $\gamma \in w_{i-1}(\alpha)$ such that $\beta \in h(\gamma)$. Since $\beta \in k(\alpha)$ and $k(\alpha) \unlhd \Phi^{+}$we get $\gamma \in k(\alpha)$, which is impossible by induction. This proves $w(\alpha) \cap k(\alpha)=\emptyset$.

We now show that $m(\alpha):=\Phi^{+} \backslash w(\alpha)$ is a normal closed pattern. Let $\beta \in m(\alpha)$ and $\gamma \in \Phi^{+}$ with $\beta+\gamma \in \Phi^{+}$. If $\beta+\gamma \in w(\alpha)$ then $\beta \prec \beta+\gamma \preceq \alpha$. Hence $\beta \preceq \alpha$ and $\beta \in w(\alpha)$, a contradiction. Thus $m(\alpha)$ is a normal closed pattern not containing $\alpha$. Since $\Sigma:=\{\alpha\}$ is representable the maximality property of $k(\alpha)=k(\{\alpha\})$ in Remark $2($ a) implies that $m(\alpha) \subseteq$ $k(\alpha)$ and therefore $\Phi^{+} \subseteq w(\alpha) \cup k(\alpha)$.

By analogy with $k(\alpha)$ and $k(\Sigma)$ we want to replace the root $\alpha$ in $w(\alpha)$ by arbitrary representable sets $\Sigma$.

Definition 11. For subsets $\Sigma \subseteq \Phi^{+}$we define $w(\Sigma):=\bigcup_{\alpha \in \Sigma} w(\alpha)$.

In particular, we have $w(\{\alpha\})=w(\alpha)$. Statement (b) in the following proposition generalizes Lemma 5.6 to arbitrary representable sets $\Sigma$.

Proposition 5.7. If $\Sigma \subseteq \Phi^{+}$is representable then

(a) $k(\Sigma)=\bigcap_{\alpha \in \Sigma} k(\alpha)$,

(b) $w(\Sigma) \cap k(\Sigma)=\emptyset$ and $w(\Sigma) \cup k(\Sigma)=\Phi^{+}$.

Proof. Suppose that $\beta \in w(\Sigma) \cap k(\Sigma)$. Then there is $\alpha \in \Sigma$ such that $\beta \in w(\alpha)$ and hence $\beta \prec \alpha$. By Lemma 2.2 there are roots $\gamma_{i} \in \Phi^{+}$such that $\beta=\gamma_{0} \prec \gamma_{1} \prec \gamma_{2} \prec \ldots \prec \gamma_{s-1} \prec$ $\gamma_{s}=\alpha$ and $\gamma_{i}-\gamma_{i-1}$ is a simple root for all $i$. It follows that the roots $\gamma_{i}$ belong to the normal closure $M$ of $\{\beta\}$ in $\Phi^{+}$. Since $\beta \in k(\Sigma)$ and $k(\Sigma) \unlhd \Phi^{+}$we get $\alpha=\gamma_{s} \in M \subseteq k(\Sigma)$. Thus $\alpha \in \Sigma \cap k(\Sigma)$, a contradiction to Remark 2(a). Hence $w(\Sigma) \cap k(\Sigma)=\emptyset$. 
From Definition 11 and Lemma 5.6 we get

$$
k(\Sigma) \subseteq \Phi^{+} \backslash w(\Sigma)=\Phi^{+} \backslash\left(\bigcup_{\alpha \in \Sigma} w(\alpha)\right)=\bigcap_{\alpha \in \Sigma}\left(\Phi^{+} \backslash w(\alpha)\right)=\bigcap_{\alpha \in \Sigma} k(\alpha) .
$$

For all $\alpha \in \Sigma$ we have $k(\alpha) \unlhd \Phi^{+}$and $\alpha \notin k(\alpha)$. Thus we have $\bigcap_{\alpha \in \Sigma} k(\alpha) \unlhd \Phi^{+}$and $\left(\bigcap_{\alpha \in \Sigma} k(\alpha)\right) \cap \Sigma=\emptyset$. From Remark 2(a) we can conclude $\bigcap_{\alpha \in \Sigma} k(\alpha) \subseteq k(\Sigma)$. Hence we have equality in (5.1) and claims (a) and (b) follow.

In the following we give an interpretation of representable sets in terms of the root poset $\left(\Phi^{+}, \preceq\right)$. This allows us to deduce the number of representable sets of size $k$ for all $k$ from results in $[9]$ and references therein. Recall that $\Phi$ is an irreducible root system of rank $n$. An antichain of the poset $\left(\Phi^{+}, \preceq\right)$ is a subset $A \subseteq \Phi^{+}$such that the elements of $A$ are pairwise incomparable. A variation of the next proposition for pattern subgroups if $\Phi$ is of type $A_{n}$ was obtained by Isaacs in [26, Theorem 3.1].

Proposition 5.8. For a subset $\Sigma \subseteq \Phi^{+}$the following are equivalent:

(a) $\Sigma$ is representable;

(b) $\Sigma$ is an antichain of the root poset $\left(\Phi^{+}, \preceq\right)$.

Proof. (a) $\Rightarrow$ (b) Suppose that $\Sigma$ is a representable set and that $\alpha, \beta \in \Sigma$ with $\alpha \neq \beta$. We need to show that $\alpha$ and $\beta$ are not comparable, so suppose otherwise. Without loss we may assume $\alpha \prec \beta$. The remarks after Definition 7 and Lemma 5.3 imply that $\beta \in n(\alpha) \subseteq k(\Sigma)$, hence $k(\Sigma) \cap \Sigma \neq \emptyset$, contradicting Remark 2(a). Thus $\alpha$ and $\beta$ are incomparable, showing that every representable set is an antichain.

(b) $\Rightarrow$ (a) Let $\Sigma$ be an antichain. We claim that $N:=\Phi^{+} \backslash w(\Sigma)$ is a normal closed pattern. Let $\alpha \in \Phi^{+}$and $\beta \in N$ with $\alpha+\beta \in \Phi^{+}$. If $\alpha+\beta \notin N$ then $\alpha+\beta \in w(\Sigma)$. Hence there is $\gamma \in \Sigma$ such that $\alpha+\beta \in w(\gamma)$. By Lemma 5.5 we have $\beta \preceq \alpha+\beta \preceq \gamma$. Thus $\beta \preceq \gamma$ and then $\beta \in w(\gamma) \subseteq w(\Sigma)$, which is impossible since $\beta \in N=\Phi^{+} \backslash w(\Sigma)$. This shows that $N \unlhd \Phi^{+}$.

Let $\alpha$ be a maximal element of $w(\Sigma)$ with respect to $\preceq$. The definition of $w(\Sigma)$ and Lemma 5.5 imply that $\alpha \in \Sigma$. Conversely, let $\alpha \in \Sigma$ and suppose that there is $\gamma \in w(\Sigma)$ such that $\alpha \prec \gamma$. By the definition of $w(\Sigma)$ there is $\beta \in \Sigma$ such that $\gamma \in w(\beta)$ and hence $\gamma \preceq \beta$ by Lemma 5.5. It follows that $\alpha \prec \beta$, contradicting the fact that $\Sigma$ is an antichain. Thus $\alpha$ is a maximal element of $w(\Sigma)$. This shows that the elements of $\Sigma$ are exactly the maximal elements of $w(\Sigma)$. We get from Lemma 3.4 that $\Sigma=\operatorname{rz}\left(U Y_{n} / P(N)\right)$, so that condition (b) of Lemma 5.1 is satisfied. Hence $\Sigma$ is representable.

We can now apply results of Cellini and Papi and of Shi on the number of antichains in root posets.

Proposition 5.9. Let $N_{k}(\Phi):=\mid\left\{\Sigma \subseteq \Phi^{+} \mid \Sigma\right.$ is representable and $\left.|\Sigma|=k\right\} \mid$ be the number of representable subsets of size $k$ of $\Phi^{+}$and $N(\Phi):=\sum_{k} N_{k}(\Phi)$ the number of all representable subsets of $\Phi$. Then:

(a) $N_{k}(\Phi)=0$ for all $k>n$, that is, $|\Sigma| \leqslant n$ for all representable subsets $\Sigma$ of $\Phi^{+}$;

(b) for all $k$ the number $N_{k}(\Phi)$ is the coefficient of $t^{k}$ in the polynomial $N(\Phi, t)$, where $N(\Phi, t)$ is given by Table 1 ;

(c) $N(\Phi)=\prod_{i=1}^{n}\left(e_{i}+h+1\right) /\left(e_{i}+1\right)$, where $e_{1}, e_{2}, \ldots, e_{n}$ are the exponents of the Weyl group $W$ of $\Phi$ and $h$ is the Coxeter number of $W$. The numbers $N(\Phi)$ for the various root systems are given by Table 2 .

Proof. (c) We know from Proposition 5.8 that $N_{k}(\Phi)$ is the number of antichains of size $k$ of $\left(\Phi^{+}, \preceq\right)$. The antichains of $\left(\Phi^{+}, \preceq\right)$ are precisely given by Cellini and Papi in [5]. They also 
obtain the closed formula for the number $N(\Phi)$. We note that the numbers $N(\Phi)$ were also obtained by Shi [34] on a case-by-case basis. This is part (c).

(a), (b) The Narayana polynomials and their coefficients were obtained by Panyushev in [33]; this is part (b) and also gives part (a).

The numbers $N_{k}(\Phi)$ are called (generalized) Narayana numbers in Fomin and Reading [9]. The numbers $N(\Phi)$ seem to have wider significance. For example, they appeared in a paper by Djoković [8] on the enumeration of conjugacy classes of elements of finite order in compact and complex semisimple Lie groups. Incidentally, the numbers $N(\Phi)$ also count clusters in cluster algebras and related objects; see [9] and the references therein. For other interesting connections between classical combinatorial objects and the characters of $U A_{n}(q)$, see Marberg [29].

TABLE 1. Generating functions $N(\Phi, t)$ for the numbers $N_{k}(\Phi)$.

$$
\begin{aligned}
& N\left(A_{n}, t\right)=\sum_{k=0}^{n} \frac{1}{n+1}\left(\begin{array}{c}
n+1 \\
k
\end{array}\right)\left(\begin{array}{l}
n+1 \\
k+1
\end{array}\right) t^{k} \\
& N\left(B_{n}, t\right)=\sum_{k=0}^{n}\left(\begin{array}{l}
n \\
k
\end{array}\right)^{2} t^{k} \\
& N\left(C_{n}, t\right)=\sum_{k=0}^{n}\left(\begin{array}{l}
n \\
k
\end{array}\right)^{2} t^{k} \\
& N\left(D_{n}, t\right)=1+t^{n}+\sum_{k=1}^{n-1}\left[\left(\begin{array}{l}
n \\
k
\end{array}\right)^{2}-\frac{n}{n-1}\left(\begin{array}{c}
n-1 \\
k-1
\end{array}\right)\left(\begin{array}{c}
n-1 \\
k
\end{array}\right)\right] t^{k} \\
& N\left(E_{6}, t\right)=1+36 t+204 t^{2}+351 t^{3}+204 t^{4}+36 t^{5}+t^{6} \\
& N\left(E_{7}, t\right)=1+63 t+546 t^{2}+1470 t^{3}+1470 t^{4}+546 t^{5}+63 t^{6}+t^{7} \\
& N\left(E_{8}, t\right)=1+120 t+1540 t^{2}+6120 t^{3}+9518 t^{4}+6120 t^{5}+1540 t^{6}+120 t^{7}+t^{8} \\
& N\left(F_{4}, t\right)=1+24 t+55 t^{2}+24 t^{3}+t^{4} \\
& N\left(G_{2}, t\right)=1+6 t+t^{2}
\end{aligned}
$$

TABLE 2. The numbers $N(\Phi)$.

\begin{tabular}{cccccccc}
\hline$A_{n}$ & $B_{n}, C_{n}$ & $D_{n}$ & $E_{6}$ & $E_{7}$ & $E_{8}$ & $F_{4}$ & $G_{2}$ \\
\hline$\frac{1}{n+2}\left(\begin{array}{c}2 n+2 \\
n+1\end{array}\right)$ & $\left(\begin{array}{c}2 n \\
n\end{array}\right)$ & $\frac{3 n-2}{n}\left(\begin{array}{c}2 n-2 \\
n-1\end{array}\right)$ & 833 & 4160 & 25080 & 105 & 8 \\
\hline
\end{tabular}

\section{Single root characters}

We keep the setting from the previous sections. In particular, $q$ is a power of a prime $p$ and $Y_{n}(q)$ is an untwisted Chevalley group defined over $\mathbb{F}_{q}$ with an irreducible root system $\Phi$. We assume throughout this section that Hypothesis 1 holds. 
For $N=\Phi^{+}$we have $\operatorname{rz}\left(U Y_{n} / P(N)\right)=\emptyset$. It follows from Lemma 5.1 that $\emptyset$ is representable and that $\operatorname{Irr}\left(U Y_{n}\right)_{\emptyset}=\left\{\mathbf{1}_{U Y_{n}}\right\}$.

In what follows we investigate $\operatorname{Irr}\left(U Y_{n}\right)_{\Sigma}$ when $|\Sigma|=1$. We have already seen in Lemma 5.4 that $\{\alpha\}$ is representable for all $\alpha \in \Phi^{+}$and we gave a recursive description of $k(\{\alpha\})=k(\alpha)$ in Definition 8.

Definition 12. For $\alpha \in \Phi^{+}$we set $\operatorname{Irr}\left(U Y_{n}\right)_{\alpha}:=\operatorname{Irr}\left(U Y_{n}\right)_{\{\alpha\}}$. We say that a character $\chi \in \operatorname{Irr}\left(U Y_{n}\right)_{\alpha}$ is a single root character lying over $\alpha$ and that it is almost faithful at $\alpha$. A midafi character (or just midafi) for $\alpha$ is a character $\chi \in \operatorname{Irr}\left(U Y_{n}\right)_{\alpha}$ such that $\chi(1)=$ $\min \left\{\psi(1) \mid \psi \in \operatorname{Irr}\left(U Y_{n}\right)_{\alpha}\right\}$. We set

$$
\operatorname{Irr}^{\text {mida }}\left(U Y_{n}\right)_{\alpha}:=\left\{\chi \in \operatorname{Irr}\left(U Y_{n}\right)_{\alpha} \mid \chi \text { is a midafi for } \alpha\right\} \text {. }
$$

Note that $q$ is fixed in Definition 12. The term midafi is an abbreviation for minimal degree almost faithful irreducible. The origin of this terminology is the thesis [27] of the second author where the term was first used in the case where $\Phi$ has Dynkin type $A_{n}$. To study single root characters we will use the concept of arms and legs.

Definition 13. Let $\alpha \in \Phi^{+}$and $a(\alpha), \ell(\alpha) \subseteq h(\alpha)$.

(a) We say that $a(\alpha)$ is an arm and $\ell(\alpha)$ is the corresponding leg of $h(\alpha)$ if the hook $h(\alpha)$ is the disjoint union $h(\alpha)=\{\alpha\} \cup a(\alpha) \cup \ell(\alpha)$ and

(1) $|a(\alpha)|=|\ell(\alpha)|$ and

(2) for each $\beta \in a(\alpha)$ there is a unique $\gamma \in \ell(\alpha)$ with $\beta+\gamma=\alpha$.

(b) If $a(\alpha)$ is an arm of $h(\alpha)$ we call the set $s(\alpha):=\Phi^{+} \backslash a(\alpha)$ of roots the source corresponding to $\alpha$. If additionally $s(\alpha)$ is a closed pattern then we call $S_{\alpha}:=P(s(\alpha))$, the source group corresponding to $\alpha$.

The terminology of hooks, arms and legs is motivated by the case where $\Phi$ is an irreducible root system of type $A_{n}$; see $[\mathbf{2 1}, \S 3.3]$ and $[\mathbf{2 7}, \S 2.3]$.

Note that Lemma 5.6 implies that we always have $k(\alpha) \subseteq s(\alpha)$. We point out that the definition above allows for $2^{m}$ choices for the set $a(\alpha)$, where $m=(|h(\alpha)|-1) / 2$. Relative to a choice of $a(\alpha)$ the sets $\ell(\alpha)$ and $s(\alpha)$ are unique. We discuss this in more detail at the end of this section. The next lemma is needed for the classification of the midafi characters when $U Y_{n}$ is of exceptional type and $n \geqslant 4$.

Definition 14. Let $\alpha \in \Phi^{+}$. We call a subset $a^{\prime}(\alpha)$ of a subhook $h^{\prime}(\alpha)$ of $h(\alpha)$ an arm of $h^{\prime}(\alpha)$ if there is an arm $a(\alpha)$ of $h(\alpha)$ with $a^{\prime}(\alpha)=a(\alpha) \cap h^{\prime}(\alpha)$. In this case we call $\ell^{\prime}(\alpha):=h^{\prime}(\alpha) \backslash\left(a^{\prime}(\alpha) \cup\{\alpha\}\right)$ the corresponding leg of $h^{\prime}(\alpha)$.

The following technical lemma will be used in $\S 8$. A stronger version for type $A_{n}$ is implicitly contained in [27]. Recall that a $p$-group $H$ is special if $Z(H)=[H, H]=\Phi(H)$. If $H$ is special with $|H|=q^{2 m+1}$ and $Z(H)$ is elementary abelian of order $q=p^{a}$, then we say that $H$ is special of type $q^{1+2 m}$.

Lemma 6.1. Let $\alpha, \beta \in \Phi^{+}$. Let $h(\alpha)$ be the hook corresponding to $\alpha$ with arm $a(\alpha)$ and leg $\ell(\alpha)$, let $h^{\prime}(\beta)$ be a subhook of $h(\beta)$ with arm $a^{\prime}(\beta)$ and leg $\ell^{\prime}(\beta)$ and let $h_{\alpha \beta}^{\prime}$ be the closed pattern generated by $h(\alpha) \cup h^{\prime}(\beta) \cup k(\alpha)$. We define $\ell_{\alpha \beta}^{\prime}:=\{\alpha, \beta\} \cup \ell(\alpha) \cup k(\alpha)$. Assume that the following hold:

(a) $h(\alpha) \cup k(\alpha), h^{\prime}(\beta) \cup k(\alpha), \Phi^{+} \backslash a(\alpha)$ are closed patterns;

(b) $h_{\alpha \beta}^{\prime} \backslash a(\alpha)$ normalizes $\ell(\alpha) \cup k(\alpha)$;

(c) $(h(\alpha) \cup k(\alpha)) \cap h^{\prime}(\beta)=\emptyset$; 
(d) the group $H_{\alpha}:=P(h(\alpha) \cup k(\alpha)) / P(k(\alpha))$ is special of type $q^{1+2|a(\alpha)|}$ with $\left[x, H_{\alpha}\right]=$ $Z\left(H_{\alpha}\right)$ for all $x \in H_{\alpha} \backslash Z\left(H_{\alpha}\right)$;

(e) the group $H_{\beta}^{\prime}:=P\left(h^{\prime}(\beta) \cup k(\alpha)\right) / P(k(\alpha))$ is special of type $q^{1+2\left|a^{\prime}(\beta)\right|}$ with $\left[x, H_{\beta}^{\prime}\right]=$ $Z\left(H_{\beta}^{\prime}\right)$ for all $x \in H_{\beta}^{\prime} \backslash Z\left(H_{\beta}^{\prime}\right)$.

Set $H_{\alpha \beta}^{\prime}:=P\left(h_{\alpha \beta}^{\prime}\right) / P(k(\alpha))$ and $L_{\alpha \beta}^{\prime}:=P\left(\ell_{\alpha \beta}^{\prime}\right) / P(k(\alpha))$ and let $\mu \in \operatorname{Irr}\left(L_{\alpha \beta}^{\prime}\right)$ with $X_{\alpha}, X_{\beta} \not$ $\operatorname{Ker}(\mu)$ and $\prod_{\gamma \in \ell(\alpha)} X_{\gamma} \subseteq \operatorname{Ker}(\mu)$. Then each $\psi \in \operatorname{Irr}\left(H_{\alpha \beta}^{\prime}, \mu\right)$ has degree $\psi(1) \geqslant q^{|a(\alpha)|+\left|a^{\prime}(\beta)\right|}$.

Proof. To apply the reduction lemma (Lemma 2.1) we introduce the following notation (only for this proof):

- $U:=H_{\alpha \beta}^{\prime}$,

- $H:=P\left(h_{\alpha \beta}^{\prime} \backslash a(\alpha)\right) / P(k(\alpha))$,

- $X:=\prod_{\gamma \in a(\alpha)} X_{\gamma}$,

- $Y:=P(\ell(\alpha) \cup k(\alpha)) / P(k(\alpha))$,

- $Z:=X_{\alpha}$,

- $\lambda:=\mu_{Z}$ and $\tilde{\lambda}:=\mu_{Z Y}$.

Since $k(\alpha)$ is a normal closed pattern $U$ is a quotient pattern group and it follows from (a) that $H$ is a subgroup of $U$. We know from Lemma 5.4 and Definition 6(a) that $Z$ is a subgroup of $U$ with $Z \subseteq Z(U)$. It follows from assumption (b) that $Y \unlhd H$ and we have $Z \cap Y=\{1\}$. By (2.1) the set $X$ is a set of representatives for $U / H$. From assumption (b) and Lemma 3.3 we get $Z Y \unlhd U$. Since $\prod_{\gamma \in \ell(\alpha)} X_{\gamma} \subseteq \operatorname{Ker}(\mu)$ we have $Y \subseteq \operatorname{Ker}(\tilde{\lambda})$ and by (a), (d) we have ${ }^{u} \tilde{\lambda} \neq \tilde{\lambda}$ for all $u \in X \backslash\{1\}$. We also have $|X|=|Y|$. Thus we can apply Lemma 2.1.

Let $\psi \in \operatorname{Irr}\left(H_{\alpha \beta}^{\prime}, \mu\right)$. We consider the restriction $\psi_{H}$ of $\psi$ to $H$. Since $Y \unlhd H$ the character $\psi_{H}$ has a constituent $\chi \in \operatorname{Irr}(H / Y, \lambda)$ with $X_{\beta} \nsubseteq \operatorname{Ker}(\chi)$. We define $H_{\beta}^{\prime}:=$ $P\left(h^{\prime}(\beta) \cup k(\alpha)\right) / P(k(\alpha))$. Note that assumption (c) implies that $H_{\beta}^{\prime}$ is a subgroup of $H$. It follows from assumption (e) that $\chi(1)=\chi_{H_{\beta}^{\prime}}(1) \geqslant q^{\left|a^{\prime}(\beta)\right|}$. By Frobenius reciprocity $\psi$ is a constituent of $\chi^{U}$. Since $\chi^{U}$ is irreducible by Lemma 2.1 we have $\psi=\chi^{U}$ and hence $\psi(1)=\chi^{U}(1)=q^{|a(\alpha)|} \cdot \chi(1) \geqslant q^{|a(\alpha)|+\left|a^{\prime}(\beta)\right|}$.

Using the theory developed in $\S \S 2-6$ we can now study the single root characters of $U Y_{n}$ for all irreducible root systems $\Phi$. In the following two sections we give a proof of Theorems 1.11.3 on a case-by-case basis and construct the midafi characters in $\operatorname{Irr}\left(U Y_{n}\right)_{\alpha}$ for all Dynkin types $Y$, all ranks $n$ and all positive roots $\alpha$. We now outline our approach; details will be given in $\S \S 7$ and 8.

Let $\Phi$ be an irreducible root system and $\alpha \in \Phi^{+}$. By construction, the size of the corresponding hook is of the form $|h(\alpha)|=1+2 k$ for some non-negative integer $k$. Hence, there are $2^{k}$ possible choices for the arm $a(\alpha)$ and the leg $\ell(\alpha)$, and in general we could not find a canonical choice. An important fact, which will be proved in $\S \S 7$ and 8 , is that one can always choose an $\operatorname{arm} a(\alpha)$ of $h(\alpha)$ with the following property:

(1) the source $s(\alpha)=\Phi^{+} \backslash a(\alpha)$ is a closed pattern.

In this case we can work with the source group $S_{\alpha}=P(s(\alpha))$ and it follows from Lemma 5.6 that $\ell(\alpha) \cup k(\alpha) \subseteq s(\alpha)$. For us a good choice for $a(\alpha)$ is one in which condition (1) and the condition

(2) the set $\ell(\alpha) \cup k(\alpha)$ is a normal closed subpattern of $s(\alpha)$

are met. If for a root $\alpha \in \Phi^{+}$and for some choice of $a(\alpha)$ we achieve conditions (1) and (2), then we define

$$
\bar{T}_{\alpha}:=S_{\alpha} / P(\{\alpha\} \cup \ell(\alpha) \cup k(\alpha)) .
$$

We now show that conditions (1) and (2), together with conditions (3) and (4) below, suffice to establish the description of $\operatorname{Irr}\left(U Y_{n}\right)_{\alpha}$ in terms of character correspondences as stated in Theorems 1.1 and 1.3 . 
Proposition 6.2. Let $\Phi$ be an irreducible root system of type $Y_{n}$. Suppose that for some root $\alpha \in \Phi^{+}$of $\operatorname{ht}(\alpha)>1$ and some choice of $a(\alpha)$ the following are true:

(1) the source $s(\alpha)$ is a closed pattern;

(2) $\ell(\alpha) \cup k(\alpha) \unlhd s(\alpha)$;

(3) $h(\alpha) \cup k(\alpha)$ is a closed pattern and $a(\alpha)$ normalizes $\{\alpha\} \cup \ell(\alpha) \cup k(\alpha)$;

(4) for each element $y \in\left(\prod_{\gamma \in a(\alpha)} X_{\gamma}\right) \backslash\{1\}$ there is some root $\beta \in \ell(\alpha)$ such that $\left\{\left[y, x_{\beta}(t)\right] \mid\right.$ $\left.t \in \mathbb{F}_{q}\right\}=X_{\alpha}$.

Then the map $\Psi_{\alpha}: \operatorname{Irr}\left(\bar{T}_{\alpha}\right) \times \operatorname{Irr}\left(X_{\alpha}\right)^{*} \rightarrow \operatorname{Irr}\left(U Y_{n}\right)_{\alpha}$, with

$$
(\mu, \lambda) \mapsto\left(\operatorname{Infl}_{\bar{T}_{\alpha}}^{S_{\alpha}} \mu \cdot \operatorname{Infl}_{X_{\alpha}}^{S_{\alpha}} \lambda\right)^{U Y_{n}}
$$

is a one-to-one correspondence with the property $\Psi_{\alpha}(\mu, \lambda)(1)=q^{|a(\alpha)|} \cdot \mu(1)$. Moreover,

$$
S_{\alpha} / P(\ell(\alpha) \cup k(\alpha)) \cong \bar{T}_{\alpha} \times X_{\alpha}
$$

Proof. We apply the reduction lemma. The role of $U$ is played by the quotient pattern group $U Y_{n} / P(k(\alpha))$ and the role of $H$ is played by $S_{\alpha} / P(k(\alpha))$. The role of $X$ is played by $\prod_{\gamma \in a(\alpha)} X_{\gamma}$, the role of $Y$ is played by $\prod_{\gamma \in \ell(\alpha)} X_{\gamma}$ and the role of $Z$ is played by $X_{\alpha}$, where we identify the root subgroups $X_{\gamma}$ with their images in $U Y_{n} / P(k(\alpha))$.

Condition (a) of Lemma 2.1 is satisfied by Definition 6(a) and Lemma 5.4. Hypothesis (2) implies that condition (b) of Lemma 2.1 is satisfied. Combining hypotheses (2) and (3) implies that $a(\alpha)$ normalizes $\{\alpha\} \cup \ell(\alpha) \cup k(\alpha)$ and $\{\alpha\} \cup \ell(\alpha) \cup k(\alpha) \unlhd s(\alpha)$. As $\Phi^{+}=s(\alpha) \cup a(\alpha)$ we now see that condition (d) of Lemma 2.1 is satisfied. Conditions (c) and (f) in Lemma 2.1 hold by the definition of $a(\alpha)$ and $\ell(\alpha)$.

We still have to check condition (e) of Lemma 2.1. Let $\lambda \in \operatorname{Irr}\left(X_{\alpha}\right)^{*}$ and let $\tilde{\lambda}$ be the inflation of $\lambda$ to $Z Y=X_{\alpha} \times P(\ell(\alpha) \cup k(\alpha)) / P(k(\alpha))$. Suppose that there is $1 \neq x \in\left(\prod_{\gamma \in a(\alpha)} X_{\gamma}\right) \backslash\{1\}$ such that ${ }^{x} \widetilde{\lambda}=\widetilde{\lambda}$. Because the linear character $\lambda$ is non-trivial on $X_{\alpha}$ there is some $t \in \mathbb{F}_{q}$ such that $\widetilde{\lambda}\left(x_{\alpha}(t)\right)=\lambda\left(x_{\alpha}(t)\right) \neq 1$. By hypothesis (4) there exist $\beta \in \ell(\alpha)$ and $t^{\prime} \in \mathbb{F}_{q}$ such that $\left[x, x_{\beta}\left(t^{\prime}\right)^{-1}\right]=x_{\alpha}(t)$. Hence

$$
{ }^{x} \widetilde{\lambda}\left(x_{\beta}\left(t^{\prime}\right)\right)=\widetilde{\lambda}\left(x^{-1} x_{\beta}\left(t^{\prime}\right) x x_{\beta}\left(t^{\prime}\right)^{-1} x_{\beta}\left(t^{\prime}\right)\right)=\widetilde{\lambda}\left(x_{\alpha}(t)\right) \widetilde{\lambda}\left(x_{\beta}\left(t^{\prime}\right)\right) \neq \widetilde{\lambda}\left(x_{\beta}\left(t^{\prime}\right)\right),
$$

contradicting ${ }^{x} \tilde{\lambda}=\tilde{\lambda}$. Thus we have ${ }^{x} \tilde{\lambda} \neq \tilde{\lambda}$ for all $x \in\left(\prod_{\gamma \in a(\alpha)} X_{\gamma}\right) \backslash\{1\}$. Now the correspondence follows from Lemma 2.1 .

Finally, we observe that hypotheses (1) and (2), combined with Lemmas 3.3 and 5.1, imply that

$$
S_{\alpha} / P(\ell(\alpha) \cup k(\alpha)) \cong \bar{T}_{\alpha} \times X_{\alpha},
$$

completing the proof.

We remark that generally hypotheses (3) and (4) are easily verified and do not present a serious obstacle.

If $\Phi$ is a classical root system, that is, $\Phi$ is of type $A_{n}, B_{n}, C_{n}$ or $D_{n}$, then we show in $\S 7$ that it is always possible to make a choice for the arm of a root such that the hypotheses of Proposition 6.2 are satisfied and for these Dynkin types we are able to derive some information on the structure of $\bar{T}_{\alpha}$.

However, when $\Phi$ is of exceptional type and rank at least 4, then there exist roots in $\Phi$ for which no choice of the arm achieves the hypotheses of Proposition 6.2. For the exceptional types of root systems one can always choose $a(\alpha)$ such that hypothesis (1) holds, but then, in general, $\ell(\alpha) \cup k(\alpha)$ is no longer normal in $s(\alpha)$. In this case we replace $\ell(\alpha)$ by a subset $\bar{\ell}(\alpha) \supsetneq \ell(\alpha)$ such that $\bar{\ell}(\alpha) \cup k(\alpha) \unlhd s(\alpha),\{\alpha\} \cup \bar{\ell}(\alpha) \cup k(\alpha) \unlhd \Phi^{+}$and such that the quotient 
pattern group $P(\bar{\ell}(\alpha) \cup k(\alpha)) / P(k(\alpha))$ is abelian. Among all choices of the arm $a(\alpha)$ such that condition (1) holds and such that $\bar{\ell}(\alpha)$ has the properties just described we take one such that $|\bar{\ell}(\alpha)|$ is minimal. For this choice we show, by restriction to a suitable subgroup, that each $\chi \in \operatorname{Irr}\left(U Y_{n}\right)_{\alpha}$ with $\bar{\ell}(\alpha) \backslash \ell(\alpha) \nsubseteq \mathbb{\operatorname { r k }}(\chi)$ has degree greater than $q^{|a(\alpha)|}$. Lemma 2.1 then gives a one-to-one correspondence

$$
\Psi: \operatorname{Irr}^{\operatorname{lin}}\left(\bar{T}_{\alpha}\right) \times \operatorname{Irr}\left(X_{\alpha}\right)^{*} \rightarrow \operatorname{Irr}^{\operatorname{mida}}\left(U Y_{n}\right)_{\alpha},
$$

where $\bar{T}_{\alpha}:=S_{\alpha} / P(\{\alpha\} \cup \bar{\ell}(\alpha) \cup k(\alpha))$. Hence for all irreducible root systems $\Phi$ (classical and exceptional) and all positive roots $\alpha$ we obtain a one-to-one correspondence

$$
\Psi: \operatorname{Irr}^{\operatorname{lin}}\left(\bar{T}_{\alpha}\right) \times \operatorname{Irr}\left(X_{\alpha}\right)^{*} \rightarrow \operatorname{Irr}^{\text {mida }}\left(U Y_{n}\right)_{\alpha} .
$$

This leads to a construction of all midafis in $\operatorname{Irr}\left(U Y_{n}\right)_{\alpha}$ and allows us to compute their number and their degrees if $\Phi$ is of exceptional type. For each positive root $\alpha$ we call the $q-1$ irreducible characters of $U Y_{n}$ in $\Psi\left(\left\{\mathbf{1}_{\bar{T}_{\alpha}}\right\} \times \operatorname{Irr}\left(X_{\alpha}\right)^{*}\right)$ the standard midafis corresponding to $\alpha$.

\section{Hook subgroups and midafis in classical groups}

In this section we prove Theorem 1.1. We will use the explicit construction of the root systems $\Phi$ of types $A_{n}, B_{n}, C_{n}$ and $D_{n}$ given in [23, 12.1]. As before, we use the following convention to keep the notation simple. Suppose that $N \subseteq S \subseteq \Phi^{+}$are closed patterns such that $N \unlhd S$. Since for each $\gamma \in S \backslash N$ the root subgroup $X_{\gamma}$ is mapped injectively into the quotient pattern group $P(S) / P(N)$ we will often identify $X_{\gamma}$ with its image $X_{\gamma} P(N)$. We assume throughout this section that Hypothesis 1 holds.

\subsection{Type $A$}

Let $n$ be a positive integer. We construct a root system of type $A_{n}$ as in [23, $\left.\S 12.1\right]$. Let $e_{1}, e_{2}, \ldots, e_{n+1} \in \mathbb{R}^{n+1}$ be the usual orthonormal unit vectors which form a basis of $\mathbb{R}^{n+1}$. Then $\Phi:=\left\{e_{i}-e_{j} \mid 1 \leqslant i \neq j \leqslant n+1\right\}$ is a root system of type $A_{n}$ and the set $\left\{\alpha_{1}, \ldots, \alpha_{n}\right\}$, where $\alpha_{i}:=e_{i}-e_{i+1}$, is a set of simple roots. The corresponding set of positive roots is $\Phi^{+}=\left\{e_{i}-e_{j} \mid 1 \leqslant i<j \leqslant n+1\right\}$.

Let $\alpha=e_{i}-e_{j} \in \Phi^{+}$. Obviously, the hook corresponding to $\alpha$ is

$$
h(\alpha)=\{\alpha\} \cup\left\{e_{i}-e_{s}, e_{s}-e_{j} \mid i<s<j\right\} .
$$

The next lemma describes the closed patterns $n(\alpha)$ and $k(\alpha)$ for all positive roots $\alpha$.

Lemma 7.1. Let $\Phi$ be a root system of type $A_{n}$ as described above. For all positive roots $\alpha=e_{i}-e_{j}$ the following are true:

(a) $n(\alpha), k(\alpha) \unlhd \Phi^{+}$,

(b) $n(\alpha)=\left\{e_{s}-e_{t} \mid 1 \leqslant s \leqslant i\right.$ and $\left.j \leqslant t \leqslant n+1\right\} \backslash\{\alpha\}$,

(c) $k(\alpha)=\left\{e_{s}-e_{t} \mid 1 \leqslant s<i\right.$ or $\left.j<t \leqslant n+1\right\}$.

Proof. Part (a) was shown in $\S 5$.

(b) By definition $n_{0}(\alpha)=\left\{e_{i}-e_{j}\right\}$. Let $\gamma=e_{k}-e_{l} \in \Phi^{+}$. Then $\alpha+\gamma \in \Phi^{+}$if and only if $l=i$ or $k=j$. In the first case we have $\alpha+\gamma=e_{k}-e_{j}$, and in the second case we have $\alpha+\gamma=e_{i}-e_{l}$. Thus,

$$
n_{1}(\alpha)=\left\{e_{k}-e_{j} \mid 1 \leqslant k<i\right\} \cup\left\{e_{i}-e_{l} \mid j<l \leqslant n+1\right\} .
$$

Now let $\gamma=e_{s}-e_{t} \in \Phi^{+}$and $k<i$. Then $\gamma+e_{k}-e_{j} \in \Phi^{+}$if and only if $t=k$ or $s=j$. In the first case we have $\gamma+e_{k}-e_{j}=e_{s}-e_{j} \in n_{1}(\alpha)$, and in the second case we have 
$\gamma+e_{k}-e_{j}=e_{k}-e_{t}$, where $k<i$ and $t>j$. Similarly, $\gamma+e_{i}-e_{l} \in \Phi^{+}$if and only if $t=i$ or $s=l$. In the first case we have $\gamma+e_{i}-e_{l}=e_{s}-e_{l}$ where $s<i$, and $l>j$, and in the second case we have $\gamma+e_{i}-e_{l}=e_{i}-e_{t} \in n_{1}(\alpha)$. It follows that

$$
n_{2}(\alpha) \backslash n_{1}(\alpha)=\left\{e_{s}-e_{t} \mid 1 \leqslant s<i \text { and } j<t \leqslant n+1\right\} .
$$

Finally, we see that for all $\gamma \in \Phi^{+}$and $\beta \in n_{2}(\alpha) \backslash n_{1}(\alpha)$ we have $\beta+\gamma \in n_{2}(\alpha)$ and therefore $n(\alpha)=n_{1}(\alpha) \cup n_{2}(\alpha)$ and (b) follows.

(c) Let $\beta=e_{s}-e_{t} \in \Phi^{+}$where $i \leqslant s<t \leqslant j$. Then we have $\beta \in h\left(e_{i}-e_{t}\right)$ and $e_{i}-e_{t} \in h(\alpha)$ and hence $\beta \in w(\alpha)$. Hence $\left\{e_{s}-e_{t} \mid i \leqslant s<t \leqslant j\right\} \subseteq w(\alpha)$ and Lemma 5.6 implies that $k(\alpha) \subseteq\left\{e_{s}-e_{t} \mid 1 \leqslant s<i\right.$ or $\left.j<t \leqslant n+1\right\}=: M$.

We claim that $M \unlhd \Phi^{+}$. Let $\beta=e_{s}-e_{t} \in M, \gamma=e_{k}-e_{l} \in \Phi^{+}$with $\beta+\gamma \in \Phi^{+}$. By the definition of $M$ we have $s<i$ or $t>j$. Suppose that $s<i$. We have $t=k$ or $s=l$. If $t=k$ then $\beta+\gamma=e_{s}-e_{l}$ with $s<i$ and hence $\beta+\gamma \in M$. If $s=l$ then $\beta+\gamma=e_{k}-e_{t}$ with $k<l=s<i$ and again $\beta+\gamma \in M$. Suppose that $t>j$. Again we have $t=k$ or $s=l$. If $t=k$ then $\beta+\gamma=e_{s}-e_{l}$ with $l>k=t>j$ and hence $\beta+\gamma \in M$. If $s=l$ then $\beta+\gamma=e_{k}-e_{t}$ with $t>j$ and again $\beta+\gamma \in M$. Thus we have $M \unlhd \Phi^{+}$. By the definition of $M$ we have $\alpha \notin M$. Hence Remark 2(a) implies that $M \subseteq k(\alpha)$. Thus $k(\alpha)=M$ and (c) follows.

In the following we study the hooks for type $A_{n}$ more closely. We define the arm $a(\alpha)$ and the leg $\ell(\alpha)$ of $h(\alpha)$ as follows:

$$
a(\alpha):=\left\{e_{i}-e_{s} \mid i<s<j\right\} \quad \text { and } \quad \ell(\alpha):=\left\{e_{s}-e_{j} \mid i<s<j\right\} .
$$

Recall that a $p$-group $P$ is special if $\Phi(P)=[P, P]=Z(P)$ is elementary abelian or if $P$ itself is elementary abelian. As in [21] we say that $P$ is special of type $q^{1+2 a}$ if $P$ is special with $|P|=q^{2 a+1}$ and $|Z(P)|=q$.

We will show below that for each $\alpha \in \Phi^{+}$the hook $h(\alpha)$ is a closed pattern. We call the pattern subgroup $H_{\alpha}:=P(h(\alpha))$ the hook subgroup corresponding to $\alpha$.

Lemma 7.2. Let $\Phi$ be a root system of type $A_{n}$ as described at the beginning of this section. For all $\alpha=e_{i}-e_{j} \in \Phi^{+}$the following are true.

(a) The hook $h(\alpha)$, the arm $a(\alpha)$ and the leg $\ell(\alpha)$ are closed patterns.

(b) The canonical projection $\pi: U A_{n} \rightarrow U A_{n} / P(k(\alpha))$ maps the hook subgroup $H_{\alpha}=$ $P(h(\alpha))$ injectively into $U A_{n} / P(k(\alpha))$ and $\pi\left(H_{\alpha}\right)$ is normal in $U A_{n} / P(k(\alpha))$.

(c) The pattern subgroups $P(a(\alpha))$ and $P(\ell(\alpha))$ are elementary abelian.

(d) If $\operatorname{ht}(\alpha)>1$ then the hook subgroup $H_{\alpha}$ is special of type $q^{1+2(j-i-1)}$ and $\left[y, H_{\alpha}\right]=$ $Z\left(H_{\alpha}\right)=X_{\alpha}$ for all $y \in H_{\alpha} \backslash Z\left(H_{\alpha}\right)$. More specifically, for each $y \in H_{\alpha} \backslash Z\left(H_{\alpha}\right)$ there is some $\beta \in h(\alpha)$ such that $\left\{\left[y, x_{\beta}(t)\right] \mid t \in \mathbb{F}_{q}\right\}=X_{\alpha}$.

Proof. (a), (c) Let $\beta, \gamma \in h(\alpha)$. Then we have $\beta+\gamma \in \Phi^{+}$if and only if $\{\beta, \gamma\}=\left\{e_{i}-\right.$ $\left.e_{s}, e_{s}-e_{j}\right\}$ for some $i<s<j$, and in this case $\beta+\gamma=\alpha \in h(\alpha)$. In particular, we have $\beta+\gamma \notin \Phi^{+}$if $\beta, \gamma \in a(\alpha)$ or if $\beta, \gamma \in \ell(\alpha)$. This implies (a) and (c).

(b) By Lemma 5.6 we have $h(\alpha) \cap k(\alpha) \subseteq w(\alpha) \cap k(\alpha)=\emptyset$. Since $H_{\alpha}, P(k(\alpha))$ are pattern subgroups we get that the restriction of $\pi$ to $H_{\alpha}$ is injective.

Let $\beta \in h(\alpha)$ and $\gamma=e_{k}-e_{l} \in \Phi^{+}$so that $k<l$. Suppose first that $\beta=e_{i}-e_{s}$ for some $i<s \leqslant j$. Then $\beta+\gamma \in \Phi^{+}$if only if $s=k$ or $l=i$. In the first case we have $\beta+\gamma=e_{i}-e_{l} \in h(\alpha) \cup k(\alpha)$. In the second case we have $\beta+\gamma=e_{k}-e_{s} \in k(\alpha)$ because $k<i$. Now suppose that $\beta=e_{s}-e_{j}$ for some $i \leqslant s<j$. Then $\beta+\gamma \in \Phi^{+}$if only if $s=l$ or $k=j$. In the first case we have $\beta+\gamma=e_{k}-e_{j} \in h(\alpha) \cup k(\alpha)$. In the second case we have $\beta+\gamma=e_{s}-e_{l} \in k(\alpha)$ because $l>j$. This proves (b).

(d) Suppose that ht $(\alpha)>1$. We have seen in the proof of (a) and (c) that for all $\beta, \gamma \in h(\alpha)$ we have $\beta+\gamma \in \Phi^{+}$if and only if $\{\beta, \gamma\}=\left\{e_{i}-e_{s}, e_{s}-e_{j}\right\}$ for some $i<s<j$, and in this 
case $\beta+\gamma=\alpha \in h(\alpha)$. It follows from Lemmas 3.3 and 3.4 that $Z\left(H_{\alpha}\right)=\left[H_{\alpha}, H_{\alpha}\right]=X_{\alpha}$. So $H_{\alpha}$ is special. Now let $y \in H_{\alpha} \backslash Z\left(H_{\alpha}\right)$. Note that $H_{\alpha} \backslash Z\left(H_{\alpha}\right) \neq \emptyset$ since $\operatorname{ht}(\alpha)>1$. We write $y=\prod_{\gamma \in h(\alpha)} x_{\gamma}\left(t_{\gamma}\right)$ as in (2.1). Because $y \notin X_{\alpha}$ there is some $\gamma \in h(\alpha) \backslash\{\alpha\}$ such that $t_{\gamma} \neq 0$. Thus, $\beta:=\alpha-\gamma \in h(\alpha)$ and we get from Lemma 3.1(a) that $\left\{\left[y, x_{\beta}(t)\right] \mid t \in \mathbb{F}_{q}\right\}=X_{\alpha}$.

The following lemma prepares a reduction result for $\operatorname{Irr}\left(U A_{n}\right)_{\alpha}$, the set of single root characters of $U A_{n}$ corresponding to positive roots $\alpha$.

Lemma 7.3. Let $\Phi$ be a root system of type $A_{n}$ as described at the beginning of this section. For all $\alpha=e_{i}-e_{j} \in \Phi^{+}$the following are true.

(a) The source $s(\alpha)$ is a closed pattern.

(b) $\ell(\alpha) \cup k(\alpha) \unlhd s(\alpha)$.

(c) $\ell(\alpha) \cup\{\alpha\} \cup k(\alpha) \unlhd \Phi^{+}$.

Proof. (a) Let $\beta=e_{k}-e_{l}, \gamma=e_{k^{\prime}}-e_{l^{\prime}} \in s(\alpha)$. Suppose that $\beta+\gamma \in a(\alpha)$. Then $\beta+\gamma=e_{i}-e_{s}$ for some $i<s<j$. Thus $k=i$ or $k^{\prime}=i$. We can assume that $k=i$ and then $k^{\prime}=l$ and $l^{\prime}=s$. But then $\beta=e_{i}-e_{l}$ where $l=k^{\prime}<l^{\prime}=s<j$ and hence $\beta \in a(\alpha)$, a contradiction. Thus $\beta+\gamma \notin \Phi^{+}$or $\beta+\gamma \in s(\alpha)$.

(b) Let $\beta=e_{s}-e_{j} \in \ell(\alpha)$ where $i<s<j$ and let $\gamma=e_{k}-e_{l} \in s(\alpha)$. Then $\beta+\gamma \in \Phi^{+}$ if and only if $s=l$ or $k=j$. In the first case we have $\beta+\gamma=e_{k}-e_{j} \in \ell(\alpha) \cup k(\alpha)$ because $k \neq i$. In the second case we have $\beta+\gamma=e_{s}-e_{l} \in k(\alpha)$ because $l>j$. This proves (b).

(c) We know from Definition 6(a) and Lemma 5.4 that $\{\alpha\} \cup k(\alpha) \unlhd \Phi^{+}$and we have seen in (b) that $s(\alpha)$ normalizes $\ell(\alpha) \cup k(\alpha)$. By Lemmas 3.3 and 7.2(d) the arm $a(\alpha)$ normalizes $\ell(\alpha) \cup\{\alpha\}$. Since $\Phi^{+}=s(\alpha) \cup a(\alpha)$ the claim in (c) follows.

By Lemma 7.3(a) and (b) we can consider the source group $S_{\alpha}=P(s(\alpha))$ and its quotient pattern group $S_{\alpha} / P(\ell(\alpha) \cup k(\alpha))$. It follows from Lemma 3.3 that we can identify the root subgroup $X_{\alpha}$ with the quotient pattern group $S_{\alpha} / P(s(\alpha) \backslash\{\alpha\})$. Lemmas 3.3 and 5.1 imply that

$$
S_{\alpha} / P(\ell(\alpha) \cup k(\alpha)) \cong \bar{T}_{\alpha} \times X_{\alpha}
$$

where

$$
\bar{T}_{\alpha}:=P(s(\alpha) \backslash\{\alpha\}) / P(\ell(\alpha) \cup k(\alpha)) .
$$

The group $\bar{T}_{\alpha}$ is generated by the images of $X_{\gamma}$ in $U A_{n} / P(\ell(\alpha) \cup k(\alpha))$ where $\gamma$ is an element of

$$
\begin{aligned}
(s(\alpha) \backslash\{\alpha\}) \backslash(\ell(\alpha) \cup k(\alpha)) & =\Phi^{+} \backslash(a(\alpha) \cup\{\alpha\} \cup \ell(\alpha) \cup k(\alpha)) \\
& =\Phi^{+} \backslash(h(\alpha) \cup k(\alpha))=\left\{e_{s}-e_{t} \mid i<s<t<j\right\} .
\end{aligned}
$$

The set $\left\{e_{s}-e_{t} \mid i<s<t<j\right\} \subseteq \Phi^{+}$is a closed pattern, and if $j-i-2>0$ it generates a root subsystem of type $A_{j-i-2}$ of $\Phi$. Thus

$$
\bar{T}_{\alpha} \cong P\left(\left\{e_{s}-e_{t} \mid i<s<t<j\right\}\right) \cong U A_{j-i-2} .
$$

We can now give a recursive description for the single root characters of $U A_{n}$.

Proposition 7.4. Let $\Phi$ be a root system of type $A_{n}$ as described above. For each root $\alpha=e_{i}-e_{j} \in \Phi^{+}$where $1 \leqslant i<j \leqslant n$ the map $\Psi_{\alpha}: \operatorname{Irr}\left(\bar{T}_{\alpha}\right) \times \operatorname{Irr}\left(X_{\alpha}\right)^{*} \rightarrow \operatorname{Irr}\left(U A_{n}\right)_{\alpha}$ with

$$
(\mu, \lambda) \mapsto\left(\operatorname{Infl}_{\bar{T}_{\alpha}}^{S_{\alpha}} \mu \cdot \operatorname{Infl}_{X_{\alpha}}^{S_{\alpha}} \lambda\right)^{U A_{n}}
$$

is a one-to-one correspondence with the property $\Psi_{\alpha}(\mu, \lambda)(1)=q^{j-i-1} \cdot \mu(1)$. We have $\bar{T}_{\alpha} \cong$ $U A_{j-i-2}$ if $j-i-2>0$ and $\bar{T}_{\alpha}=\{1\}$ otherwise. 
Proof. The content of Lemmas 7.3 and 7.2(a) is that hypotheses (1)-(3) of Proposition 6.2 are satisfied, and the content of Lemma 7.2(d) is that hypothesis (4) of Proposition 6.2 is satisfied. This proves the correspondence.

\subsection{Type $B$}

Let $n \geqslant 2$ be an integer. We construct a root system of type $B_{n}$ as in [23, §12.1]. Let $e_{1}, e_{2}, \ldots, e_{n} \in \mathbb{R}^{n}$ be the usual orthonormal unit vectors which form a basis of $\mathbb{R}^{n}$. Then $\Phi:=\left\{ \pm\left(e_{i} \pm e_{j}\right) \mid 1 \leqslant i \neq j \leqslant n\right\} \cup\left\{ \pm e_{i} \mid 1 \leqslant i \leqslant n\right\}$ is a root system of type $B_{n}$ and the set $\left\{\alpha_{1}, \ldots, \alpha_{n}\right\}$, where $\alpha_{i}:=e_{i}-e_{i+1}$ for $i=1,2, \ldots, n-1$ and $\alpha_{n}:=e_{n}$, is a set of simple roots. The corresponding set of positive roots is $\Phi^{+}=\left\{e_{i} \pm e_{j} \mid 1 \leqslant i<j \leqslant n\right\} \cup\left\{e_{i} \mid 1 \leqslant i \leqslant n\right\}$.

The highest long root with respect to this base is $e_{1}+e_{2}$, whereas the highest short root is $e_{1}$. We note that the long roots of $\Phi$ form a $D_{n}$-subsystem. Also we recall that the Chevalley commutator relations imply that if $\alpha$ is short and $\beta$ is long with $\alpha+\beta \in \Phi^{+}$, then $\left[X_{\alpha}, X_{\beta}\right] \subseteq$ $X_{\alpha+\beta} X_{2 \alpha+\beta}$, where $\alpha+\beta$ is short and $2 \alpha+\beta$ is long, and that if both $\alpha$ and $\beta$ are short with $\alpha+\beta \in \Phi^{+}$, then $\left[X_{\alpha}, X_{\beta}\right] \subseteq X_{\alpha+\beta}$, where $\alpha+\beta$ is long.

From the explicit description of the root systems (or from the Dynkin diagram) we see that $\left\{\alpha_{2}, \alpha_{3}, \ldots, \alpha_{n}\right\}$ generates a root subsystem $\Phi_{1^{\prime}}$ of type $B_{n-1}$ and that $\left\{\alpha_{1}, \alpha_{2}, \ldots, \alpha_{n-1}\right\}$ generates a root subsystem $\Phi_{n^{\prime}}$ of type $A_{n-1}$. We set $\Phi_{1^{\prime}}^{+}:=\Phi_{1^{\prime}} \cap \Phi^{+}, \Psi_{1^{\prime}}^{+}:=\Phi^{+} \backslash \Phi_{1^{\prime}}^{+}$ and $\Phi_{n^{\prime}}^{+}:=\Phi_{n^{\prime}} \cap \Phi^{+}, \Psi_{n^{\prime}}^{+}:=\Phi^{+} \backslash \Phi_{n^{\prime}}^{+}$. The sets $\Psi_{1^{\prime}}^{+}$and $\Psi_{n^{\prime}}^{+}$are normal closed patterns. In fact, $P\left(\Psi_{1^{\prime}}^{+}\right)$and $P\left(\Psi_{n^{\prime}}^{+}\right)$are the unipotent radicals of the standard parabolic subgroups corresponding to $\left\{\alpha_{2}, \alpha_{3}, \ldots, \alpha_{n}\right\}$ and $\left\{\alpha_{1}, \alpha_{2}, \ldots, \alpha_{n-1}\right\}$, respectively. Furthermore, the sets $\Phi_{1^{\prime}}^{+}$and $\Phi_{n^{\prime}}^{+}$are closed patterns and $P\left(\Phi_{1^{\prime}}^{+}\right) \cong U B_{n} / P\left(\Psi_{1^{\prime}}^{+}\right) \cong U B_{n-1}$ and $P\left(\Phi_{n^{\prime}}^{+}\right) \cong$ $U B_{n} / P\left(\Psi_{n^{\prime}}^{+}\right) \cong U A_{n-1}$.

Let $\alpha \in \Phi^{+}$and $\chi \in \operatorname{Irr}\left(U B_{n}\right)_{\alpha}$. Suppose that $\alpha \in \Phi_{1^{\prime}}^{+}$. It follows from Lemma 5.4 and Remark $2\left(\right.$ a), (c) that $P\left(\Psi_{1^{\prime}}^{+}\right) \subseteq \operatorname{Ker}(\chi)$. Thus, we can identify $\chi$ with a single root character of $P\left(\Phi_{1^{\prime}}^{+}\right) \cong U B_{n} / P\left(\Psi_{1^{\prime}}^{+}\right) \cong U B_{n-1}$. In this way, the classification and construction of the elements of $\operatorname{Irr}\left(U B_{n}\right)_{\alpha}$ are reduced to the case $B_{n-1}$. Similarly, if $\alpha \in \Phi_{n^{\prime}}^{+}$we can identify $\chi$ with a single root character of $P\left(\Phi_{n^{\prime}}^{+}\right)$and thereby get a reduction to the case $A_{n-1}$ which has already been treated in $\S 7.1$. Hence, we only have to consider positive roots $\alpha$ which are not contained in $\Phi_{1^{\prime}}^{+} \cup \Phi_{n^{\prime}}^{+}$, that is, the roots $e_{1}+e_{i}$ where $1<i \leqslant n$ and the root $e_{1}$.

Lemma 7.5. Let $\Phi$ be a root system of type $B_{n}$ as described above. For all positive roots of the form $\alpha=e_{1}+e_{i}$ we have

$$
h(\alpha)=\left\{e_{1}+e_{i}, e_{1}, e_{i}\right\} \cup\left\{e_{1}-e_{s}, e_{s}+e_{i} \mid 1<s<i\right\} \cup\left\{e_{1} \pm e_{s}, e_{i} \mp e_{s} \mid i<s \leqslant n\right\} .
$$

Proof. First, we observe that clearly $e_{1}, e_{i} \in h\left(e_{1}+e_{i}\right)$, and that no other combination of short roots can add to $\alpha$.

Next, let $\beta=e_{s} \pm e_{t}, \gamma=e_{s^{\prime}} \in \Phi^{+}$. We see immediately that $\beta+\gamma \notin \Phi^{+}$or $\beta+\gamma$ is a short root and therefore $\beta+\gamma \neq \alpha$. Hence, $\beta, \gamma \notin h(\alpha)$.

Finally, let $\beta=e_{s} \pm e_{t}, \gamma=e_{s^{\prime}} \pm e_{t^{\prime}} \in \Phi^{+}$with $s<t$ and $s^{\prime}<t^{\prime}$ and $\beta+\gamma=\alpha$. We see immediately that one of the two \pm signs has to be a + sign and that the other one has to be a - sign and that $t=t^{\prime}$. Furthermore, we can assume $s=1$ and $s^{\prime}=i$. Hence

$$
(\beta, \gamma) \in\left\{\left(e_{1}-e_{s}, e_{s}+e_{i}\right) \mid 1<s<i \text { or } i<s \leqslant n\right\} \cup\left\{\left(e_{1}+e_{s}, e_{i}-e_{s}\right) \mid i<s \leqslant n\right\}
$$

and the claim follows.

We are now able to determine the patterns $n(\alpha)$ and $k(\alpha)$.

Lemma 7.6. Let $\Phi$ be a root system of type $B_{n}$ as described above. For all positive roots of the form $\alpha=e_{1}+e_{i}$ the following are true. 
(a) $n(\alpha), k(\alpha) \unlhd \Phi^{+}$.

(b) $n(\alpha)=\left\{e_{1}+e_{s} \mid 1<s<i\right\}$.

(c) $k(\alpha)=\left\{e_{s}+e_{t} \mid 1 \leqslant s<t<i\right\}$.

Proof. Part (a) was shown in $\S 5$.

(b) By definition $n_{0}(\alpha)=\left\{e_{1}+e_{i}\right\}$. Let $\gamma \in \Phi^{+}$. For $\gamma=e_{s}$ or $\gamma=e_{k}+e_{l}$ we have $\alpha+\gamma \notin \Phi^{+}$.

Therefore, $\alpha+\gamma \in \Phi^{+}$if and only if $\gamma=e_{s}-e_{i}$ for some $1<s<i$ and in this case we have $\alpha+\gamma=e_{1}+e_{s}$. Thus, $n_{1}(\alpha)=\left\{e_{1}+e_{s} \mid 1<s<i\right\}$. Again, if $\gamma \in \Phi^{+}$is of the form $\gamma=e_{k}$ then $\gamma+\left(e_{1}+e_{s}\right) \notin \Phi^{+}$. But if $\gamma=e_{k} \pm e_{l} \in \Phi^{+}$then $\gamma+\left(e_{1}+e_{s}\right) \in \Phi^{+}$if and only if $\gamma=e_{t}-e_{s}$ for some $1<t<s$ and in this case we have $\gamma+\left(e_{1}+e_{s}\right)=e_{1}+e_{t}$. Thus $n_{2}(\alpha) \subseteq n_{1}(\alpha)$ and then $n(\alpha)=n_{1}(\alpha)$ and (b) follows.

(c) Let $\beta=e_{s}$. If $s=1$, then $\beta \in h(\alpha) \subseteq w(\alpha)$. If $s>1$, then $\beta+\left(e_{1}-e_{s}\right)=e_{1} \in h(\alpha)$ implies that $\beta \in w(\alpha)$.

Let $\beta=e_{s}+e_{t} \in \Phi^{+}$where $s<t$ and $t \geqslant i$. If $s=1$ then $\beta \in h(\alpha) \subseteq w(\alpha)$. If $s>1$ then $\beta \in h\left(e_{1}+e_{t}\right)$ and $e_{1}+e_{t} \in h(\alpha)$, hence $\beta \in w(\alpha)$.

Now let $\beta=e_{s}-e_{t} \in \Phi^{+}$where $s<t$. If $s=1$, then $\beta \in h\left(e_{1}\right)$ and $e_{1} \in h(\alpha)$. Thus, $\beta \in w(\alpha)$. If $s>1$, then $\beta \in h\left(e_{1}-e_{t}\right)$ and $e_{1}-e_{t} \in h\left(e_{1}\right)$ and $e_{1} \in h(\alpha)$. Hence $\beta \in w(\alpha)$.

It follows from Lemma 5.6 that

$$
k(\alpha) \subseteq\left\{e_{s}+e_{t} \mid 1 \leqslant s<t<i\right\}=: M
$$

We claim that $M \unlhd \Phi^{+}$. Let $\beta=e_{s}+e_{t} \in M, \gamma \in \Phi^{+}$with $\beta+\gamma \in \Phi^{+}$. It follows that $\gamma$ is of the form $\gamma=e_{k}-e_{s}$ with $k<s<t<i$ or $\gamma=e_{k}-e_{t}$ with $k<t<i$. In the first case we have $\beta+\gamma=e_{k}+e_{t}$ with $k<t<i$. In the second case we have $\beta+\gamma=e_{k}+e_{s}$ with $k, s<i$. Thus, $\beta+\gamma \in M$ in both cases. Thus we have $M \unlhd \Phi^{+}$. By the definition of $M$ we have $\alpha \notin M$. Hence Remark 2(a) implies that $M \subseteq k(\alpha)$. Thus $k(\alpha)=M$ and (c) follows. This completes the proof.

For $\alpha=e_{1}+e_{i} \in \Phi^{+}$we define the arm $a(\alpha)$ and the leg $\ell(\alpha)$ of $h(\alpha)$ as follows:

$$
\begin{aligned}
a(\alpha) & :=\left\{e_{i}\right\} \cup\left\{e_{i} \pm e_{s} \mid i<s\right\} \cup\left\{e_{1}-e_{s} \mid 1<s<i\right\}, \\
\ell(\alpha) & :=\left\{e_{1}\right\} \cup\left\{e_{1} \pm e_{s} \mid i<s\right\} \cup\left\{e_{s}+e_{i} \mid 1<s<i\right\} .
\end{aligned}
$$

As for type $A_{n}$, we will show below that for each $\alpha \in \Phi^{+}$the hook $h(\alpha)$ is a closed pattern and call $H_{\alpha}:=P(h(\alpha))$ the hook subgroup corresponding to $\alpha$.

Lemma 7.7. Let $\Phi$ be a root system of type $B_{n}$ as described at the beginning of this section. For all $\alpha=e_{1}+e_{i} \in \Phi^{+}$the following are true.

(a) The hook $h(\alpha)$, the arm $a(\alpha)$ and the leg $\ell(\alpha)$ are closed patterns.

(b) The canonical projection $\pi: U B_{n} \rightarrow U B_{n} / P(k(\alpha))$ maps the hook subgroup $H_{\alpha}=$ $P(h(\alpha))$ injectively into $U B_{n} / P(k(\alpha))$.

(c) The pattern subgroups $P(a(\alpha))$ and $P(\ell(\alpha))$ are elementary abelian.

(d) If $\operatorname{ht}(\alpha)>1$ then the hook subgroup $H_{\alpha}$ is special of type $q^{1+2(2 n-i-1)}$ and $\left[y, H_{\alpha}\right]=$ $Z\left(H_{\alpha}\right)=X_{\alpha}$ for all $y \in H_{\alpha} \backslash Z\left(H_{\alpha}\right)$. More specifically, for each $y \in H_{\alpha} \backslash Z\left(H_{\alpha}\right)$ there is some $\beta \in h(\alpha)$ such that $\left\{\left[y, x_{\beta}(t)\right] \mid t \in \mathbb{F}_{q}\right\}=X_{\alpha}$.

Proof. (a), (c) Let $\beta, \gamma \in h(\alpha)$. Then we have $\beta+\gamma \in \Phi^{+}$if and only if $\{\beta, \gamma\}=\left\{e_{1}-\right.$ $\left.e_{s}, e_{s}+e_{i}\right\}$ for some $s \neq i$, or $\{\beta, \gamma\}=\left\{e_{1}+e_{s}, e_{i}-e_{s}\right\}$ for some $s>i$, or $\{\beta, \gamma\}=\left\{e_{1}, e_{i}\right\}$, and in all of these cases $\beta+\gamma=\alpha \in h(\alpha)$. In particular, we have $\beta+\gamma \notin \Phi^{+}$if $\beta, \gamma \in a(\alpha)$ or if $\beta, \gamma \in \ell(\alpha)$. This implies (a) and (c). 
(b) By Lemma 5.6 we have $h(\alpha) \cap k(\alpha) \subseteq w(\alpha) \cap k(\alpha)=\emptyset$. Since $H_{\alpha}, P(k(\alpha))$ are pattern subgroups we get that the restriction of $\pi$ to $H_{\alpha}$ is injective.

(d) Suppose that ht $(\alpha)>1$. We have seen in the proof of (a) and (b) that for all $\beta, \gamma \in h(\alpha)$ we have $\beta+\gamma \in \Phi^{+}$if and only if $\{\beta, \gamma\}=\left\{e_{1}-e_{s}, e_{s}+e_{i}\right\}$ for some $s \neq i$, or $\{\beta, \gamma\}=$ $\left\{e_{1}+e_{s}, e_{i}-e_{s}\right\}$ for some $s>i$, or $\{\beta, \gamma\}=\left\{e_{1}, e_{i}\right\}$, and in all of these cases $\beta+\gamma=\alpha \in h(\alpha)$. It follows from Lemmas 3.3 and 3.4 that $Z\left(H_{\alpha}\right)=\left[H_{\alpha}, H_{\alpha}\right]=X_{\alpha}$. So $H_{\alpha}$ is special. The remaining part of the proof of (d) is analogous to the proof of Lemma 7.2(d).

Lemma 7.8. Let $\Phi$ be a root system of type $B_{n}$ as described at the beginning of this section. For all $\alpha=e_{1}+e_{i} \in \Phi^{+}$the following are true.

(a) The source $s(\alpha)$ is a closed pattern.

(b) $\ell(\alpha) \cup k(\alpha) \unlhd s(\alpha)$.

(c) $\ell(\alpha) \cup\{\alpha\} \cup k(\alpha) \unlhd \Phi^{+}$.

Proof. (a) Let $\beta \in a(\alpha)$ and $\gamma, \gamma^{\prime} \in \Phi^{+}$such that $\beta=\gamma+\gamma^{\prime}$. To prove that $s(\alpha)$ is a pattern it suffices to show that $\gamma \in a(\alpha)$ or $\gamma^{\prime} \in a(\alpha)$.

Suppose that $\beta=e_{i}$. Then $\left\{\gamma, \gamma^{\prime}\right\}=\left\{e_{i}-e_{s}, e_{s}\right\}$ for some $i<s$ and hence $\gamma \in a(\alpha)$ or $\gamma^{\prime} \in a(\alpha)$. Suppose next that $\beta=e_{1}-e_{s}$ where $1<s<i$. Then $\left\{\gamma, \gamma^{\prime}\right\}=\left\{e_{1}-e_{l}, e_{l}-e_{s}\right\}$ for some $1<l<s<i$ and hence $\gamma \in a(\alpha)$ or $\gamma^{\prime} \in a(\alpha)$. Now suppose that $\beta=e_{i}+e_{s}$ where $i<s \leqslant n$. Then $\left\{\gamma, \gamma^{\prime}\right\}=\left\{e_{i}+e_{l}, e_{s}-e_{l}\right\}$ for some $i<l \leqslant n$, or $\left\{\gamma, \gamma^{\prime}\right\}=\left\{e_{s}+e_{l}, e_{i}-e_{l}\right\}$ for some $i<l \leqslant n$, or $\left\{\gamma, \gamma^{\prime}\right\}=\left\{e_{s}, e_{i}\right\}$. Hence in all three cases $\gamma \in a(\alpha)$ or $\gamma^{\prime} \in a(\alpha)$. Finally, suppose that $\beta=e_{i}-e_{s}$ where $i<s \leqslant n$. Then $\left\{\gamma, \gamma^{\prime}\right\}=\left\{e_{i}-e_{l}, e_{l}-e_{s}\right\}$ for some $i<l<s \leqslant n$ and again $\gamma \in a(\alpha)$ or $\gamma^{\prime} \in a(\alpha)$.

(b) Let $\beta \in \ell(\alpha)$ and $\gamma \in s(\alpha)$ such that $\beta+\gamma \in \Phi^{+}$. We have to show that $\beta+\gamma \in \ell(\alpha) \cup k(\alpha)$. If $\beta=e_{s}+e_{i}$ where $1<s<i$, then $\gamma$ has to be of the form $\gamma=e_{k}-e_{l}$ where $k<l$. Thus $\beta+\gamma=e_{k}+e_{i}$ where $1<k<i$, or $\beta+\gamma=e_{s}+e_{k}$ where $s, k<i$. In the first case $\beta+\gamma \in \ell(\alpha)$, and in the second case $\beta+\gamma \in k(\alpha)$. Now suppose that $\beta=e_{1}+e_{s}$ where $s>i$. Then $\gamma$ has to be of the form $\gamma=e_{k}-e_{l}$ where $k<l$, and $\beta+\gamma=e_{1}+e_{k}$ where $k \neq 1, i$. Hence $\beta+\gamma \in k(\alpha) \cup \ell(\alpha)$.

Now suppose that $\beta=e_{1}-e_{s}$ where $s>i$, and $\gamma=e_{k}+e_{l}$ where $k<l$, or $\gamma=e_{l}$. Then $\beta+\gamma=e_{1}+e_{k}$ where $k \neq 1, i$, or $\beta+\gamma=e_{1}+e_{l}$ where $l \neq 1, i$, or $\beta+\gamma=e_{1}$. Hence $\beta+\gamma \in k(\alpha) \cup \ell(\alpha)$. Finally, suppose that $\beta=e_{1}-e_{s}$ where $s>i$, and $\gamma=e_{k}-e_{l}$ where $k<l$. Then $\beta+\gamma=e_{1}-e_{l}$ where $l>i$. Thus $\beta+\gamma \in \ell(\alpha)$. Finally, suppose that $\beta=e_{1}$. Then $\beta+\gamma=e_{1}+e_{l}$ where $l \neq 1, i$. Hence $\beta+\gamma \in \ell(\alpha) \cup k(\alpha)$, proving (b).

(c) In light of (b) it suffices to prove that $a(\alpha)$ normalizes $\ell(\alpha) \cup\{\alpha\}$, and this was shown in Lemma 7.7(d). Hence (c) follows.

REMARK 3. Note that, in contrast to type $A_{n}$, we did not claim in Lemma 7.7 that $\pi\left(H_{\alpha}\right)$ is normal in $U B_{n} / P(k(\alpha))$. In fact, for all $2<i<n$ the patterns $h(\alpha)$ and $h(\alpha) \cup k(\alpha)$ are not normal because $\left(e_{2}-e_{i}\right)+\left(e_{i}-e_{n}\right) \notin h(\alpha) \cup k(\alpha)$.

We can now establish our desired correspondence for long root subgroups of root systems of type $B_{n}$.

Proposition 7.9. Let $\Phi$ be a root system of type $B_{n}$ as described above. For each $\alpha=$ $e_{1}+e_{i} \in \Phi^{+}$where $1<i \leqslant n$ the map $\Psi_{\alpha}: \operatorname{Irr}\left(\bar{T}_{\alpha}\right) \times \operatorname{Irr}\left(X_{\alpha}\right)^{*} \rightarrow \operatorname{Irr}\left(U B_{n}\right)_{\alpha}$ with

$$
(\mu, \lambda) \mapsto\left(\operatorname{Infl}_{\bar{T}_{\alpha}}^{S_{\alpha}} \mu \cdot \operatorname{Infl}_{X_{\alpha}}^{S_{\alpha}} \lambda\right)^{U B_{n}}
$$

is a one-to-one correspondence with the property $\Psi_{\alpha}(\mu, \lambda)(1)=q^{2 n-i-1} \cdot \mu(1)$. 
Proof. The content of Lemmas 7.8 and 7.7(a) is that hypotheses (1)-(3) of Proposition 6.2 are satisfied, and the content of Lemma 7.7(d) is that hypothesis (4) of Proposition 6.2 is satisfied. This proves the correspondence.

Our next result gives structural information concerning the group $\bar{T}_{\alpha}$. To this end we define closed patterns

$$
b_{i, j}:=\left\{e_{t}, e_{r} \pm e_{s} \mid r, s, t \notin\{i, j\}, \text { and } r<s\right\}
$$

where $1 \leqslant i<j \leqslant n$, and

$$
\mathrm{ob}\left(e_{1}+e_{i}\right):=\left\{e_{1}-e_{i}, \ldots, e_{i-1}-e_{i}\right\}
$$

Lemma 7.10. Let $\Phi$ be a root system of type $B_{n}$ as described at the beginning of this subsection. For all $\alpha=e_{1}+e_{i} \in \Phi^{+}$the following is true.

(a) $S_{\alpha} / P(\ell(\alpha) \cup k(\alpha)) \cong \bar{S}_{\alpha} / P(\ell(\alpha)) P(k(\alpha)) \cong \bar{T}_{\alpha} \times X_{\alpha}$.

(b) $\bar{T}_{\alpha} \cong P\left(b_{1, i}\right) / P\left(b_{1, i} \cap k(\alpha)\right) \ltimes P(\mathrm{ob}(\alpha)) / P(\mathrm{ob}(\alpha) \cap k(\alpha))$.

(c) If $i<n$ then $\operatorname{kob}(\alpha):=b_{1, i} \backslash\left\{e_{r}-e_{s} \mid 1<r<s<i\right\}$ is a closed pattern that is normal in $b_{1, i}$ and $P(\operatorname{kob}(\alpha))$ centralizes $P(\mathrm{ob}(\alpha)) / P(\mathrm{ob}(\alpha) \cap k(\alpha))$.

(d) If $i \in\{2,3\}$, then $\operatorname{kob}(\alpha)=b_{1, i}, k(\alpha)=n(\alpha)$ and

$$
\bar{T}_{\alpha} \cong U B_{n-2} \times\left(U A_{1}\right)^{i-1} .
$$

(e) If $i>3$ then $P\left(b_{1, i}\right) / P\left(b_{1, i} \cap k(\alpha)\right)$ is isomorphic to a quotient pattern group of $U B_{n-2}$.

(f) If $i>3$ then $P\left(b_{1, i}\right) / P\left(\operatorname{kob}(\alpha) \cup\left(k(\alpha) \cap b_{1, i}\right)\right) \cong U A_{i-3}$.

Proof. (a) follows from the definition of $\bar{T}_{\alpha}$ and Lemma 7.8(b) and (c).

(b) By inspection, $b_{1, i} \cap(\{\alpha\} \cup a(\alpha))=\emptyset$ and $\operatorname{ob}(\alpha) \cap(\{\alpha\} \cup a(\alpha))=\emptyset$. Hence, $b_{1, i} \cup \operatorname{ob}(\alpha) \cup$ $\ell(\alpha) \cup k(\alpha) \subseteq s(\alpha) \backslash\{\alpha\}$. Thus

$$
P\left(b_{1, i} \cup \operatorname{ob}(\alpha)\right) P(\ell(\alpha) \cup k(\alpha)) / P(\ell(\alpha) \cup k(\alpha)) \subseteq \bar{T}_{\alpha} .
$$

By definition, $\operatorname{ob}(\alpha) \cup b_{1, i} \cup k(\alpha) \cup h(\alpha)=\Phi^{+}$and therefore

$$
b_{1, i} \cup \mathrm{ob}(\alpha) \cup k(\alpha) \cup \ell(\alpha) \supseteq \Phi^{+} \backslash(\{\alpha\} \cup a(\alpha))=s(\alpha) \backslash\{\alpha\},
$$

and it follows that we have equality in (7.2). For all $\beta \in \mathrm{ob}(\alpha)$ and $\gamma \in b_{1, i}$ we have $\beta+\gamma \notin \Phi^{+}$ or $\beta+\gamma \in \mathrm{ob}(\alpha)$ and hence $P\left(b_{1, i}\right)$ normalizes $P(\mathrm{ob}(\alpha))$. By definition of ob $(\alpha)$ we have $\mathrm{ob}(\alpha) \cap b_{1, i}=\emptyset$ and thus $\bar{T}_{\alpha}$ is the semidirect product of $P\left(b_{1, i}\right) P(\ell(\alpha) \cup k(\alpha)) / P(\ell(\alpha) \cup k(\alpha))$ and $P(\operatorname{ob}(\alpha)) P(\ell(\alpha) \cup k(\alpha)) / P(\ell(\alpha) \cup k(\alpha))$. Because $b_{1, i} \cap \ell(\alpha)=\emptyset$ and $\operatorname{ob}(\alpha) \cap \ell(\alpha)=\emptyset$ we have $P\left(b_{1, i}\right) \cap P(\ell(\alpha) \cup k(\alpha))=P\left(b_{1, i} \cap k(\alpha)\right)$ and $P(\operatorname{ob}(\alpha)) \cap P(\ell(\alpha) \cup k(\alpha))=P(\operatorname{ob}(\alpha) \cap k(\alpha))$, which implies (b).

(c) The only way to write $e_{r}-e_{s}$, where $1<r<s<i$, as a sum of two positive roots is $e_{r}-e_{s}=\beta+\gamma$ where $\{\beta, \gamma\}=\left\{e_{r}-e_{t}, e_{t}-e_{s}\right\}$ for some $r<t<s$. Since $b_{1, i}$ is a closed pattern and $\beta, \gamma \notin \operatorname{kob}(\alpha)$ it follows that $\operatorname{kob}(\alpha)$ is a closed pattern which is normal in $b_{1, i}$. Suppose that $\beta=e_{r} \pm e_{r^{\prime}} \in b_{1, i}$ and $\gamma=e_{s}-e_{i} \in \mathrm{ob}(\alpha)$. Then $\beta+\gamma \in \mathrm{ob}(\alpha)$ only if $\beta=e_{r}-e_{s}$ where $1<r<s<i$, that is, if $\beta \notin \operatorname{kob}(\alpha)$. This proves (c).

(d) For $i \in\{2,3\}$ we have $\left\{e_{r}-e_{s} \mid 1<r<s<i\right\}=\emptyset$. Hence $\operatorname{kob}(\alpha)=b_{1, i}$ and the semidirect product in (b) is a direct product. Now Lemma 7.6(b) and (c) imply that $k(\alpha)=n(\alpha)$. Furthermore, $|\mathrm{ob}(\alpha)|=i-1$ and $\mathrm{ob}(\alpha) \cap k(\alpha)=\emptyset$. Since $P(\mathrm{ob}(\alpha))$ is elementary abelian we get $P(\operatorname{ob}(\alpha)) / P(\operatorname{ob}(\alpha) \cap k(\alpha)) \cong\left(U A_{1}\right)^{i-1}$. Because $b_{1, i} \cap k(\alpha)=\emptyset$ and $b_{1, i}$ generates a root subsystem of $\Phi$ of type $B_{n-2}$ we get $P\left(b_{1, i}\right) / P\left(b_{1, i} \cap k(\alpha)\right) \cong U B_{n-2}$.

(e) follows from the fact that $b_{1, i}$ generates a root subsystem of $\Phi$ of type $B_{n-2}$.

(f) By definition of $\operatorname{kob}(\alpha)$ the set $b_{1, i}$ is the disjoint union of $\operatorname{kob}(\alpha)$ and the set $\left\{e_{r}-e_{s} \mid\right.$ $1<r<s<i\}$. The latter generates a root subsystem of $\Phi$ of type $A_{i-3}$. This completes the proof. 
Finally, we consider the highest short root $\alpha:=e_{1}$. A simple calculation shows the following result.

Lemma 7.11. $h\left(e_{1}\right)=\left\{e_{1}, e_{1}-e_{i}, e_{i} \mid 2 \leqslant i \leqslant n\right\}$.

We define

$$
\begin{aligned}
& \ell\left(e_{1}\right):=\left\{e_{1}-e_{i} \mid 2 \leqslant i \leqslant n\right\}, \\
& a\left(e_{1}\right):=\left\{e_{i} \mid 2 \leqslant i \leqslant n\right\},
\end{aligned}
$$

and remark that $\ell\left(e_{1}\right)$ is a closed pattern whereas $a\left(e_{1}\right)$ is a closed pattern only modulo $k(\alpha)$.

LEMma 7.12. The following are true.

(a) $n\left(e_{1}\right)=\left\{e_{1}+e_{r} \mid 1<r \leqslant n\right\}$ and $k\left(e_{1}\right)=\left\{e_{r}+e_{s} \mid 1 \leqslant r<s \leqslant n\right\}$.

(b) $k\left(e_{1}\right)$ is a normal closed pattern in $\Phi^{+}$.

(c) $\left\{e_{1}\right\} \cup \ell\left(e_{1}\right) \cup k\left(e_{1}\right) \unlhd h\left(e_{1}\right) \cup k\left(e_{1}\right)$.

(d) The group $P\left(h\left(e_{1}\right) \cup k\left(e_{1}\right)\right) / P\left(k\left(e_{1}\right)\right)$ is special of order $q^{1+2(n-1)}$. Moreover, modulo $P\left(k\left(e_{1}\right)\right)\left[y, H_{e_{1}}\right]=Z\left(H_{e_{1}}\right)=X_{e_{1}}$ for all $y \in H_{e_{1}} \backslash Z\left(H_{e_{1}}\right)$.

(e) $s\left(e_{1}\right)$ is a closed pattern.

(f) $\ell\left(e_{1}\right) \cup k\left(e_{1}\right) \unlhd s\left(e_{1}\right)$ and $s\left(e_{1}\right) /\left(\left\{e_{1}\right\} \cup \ell\left(e_{1}\right) \cup k\left(e_{1}\right)\right)$ is a closed pattern of type $A_{n-2}$.

(g) $\bar{T}_{e_{1}} \cong U A_{n-2}, S_{e_{1}} / P\left(\ell\left(e_{1}\right) \cup k\left(e_{1}\right)\right) \cong \bar{T}_{e_{1}} \times X_{e_{1}}$.

(h) The map $\Psi_{e_{1}}: \operatorname{Irr}\left(\bar{T}_{e_{1}}\right) \times \operatorname{Irr}\left(X_{e_{1}}\right)^{*} \rightarrow \operatorname{Irr}\left(U B_{n}\right)_{e_{1}}$ with

$$
(\mu, \lambda) \mapsto\left(\operatorname{Inff}_{\bar{T}_{e_{1}}}^{S_{e_{1}}} \mu \cdot \operatorname{Infl}_{X_{e_{1}}}^{S_{e_{1}}} \lambda\right)^{U B_{n}}
$$

is a one-to-one correspondence with the property $\Psi_{e_{1}}(\mu, \lambda)(1)=q^{n-1} \cdot \mu(1)$.

Proof. (b) was shown in $\S 5$.

(a) The fact that $e_{1}+e_{r}$ is the sum of the two short roots $e_{1}$ and $e_{r}$ both of which lie in $h\left(e_{1}\right)$ shows that $n_{1}(\alpha)=\left\{e_{1}+e_{r} \mid 1<r \leqslant n\right\} \subseteq n(\alpha)$. Now if $\gamma+\nu \in \Phi^{+}$for $\gamma \in \Phi^{+}$and $\nu \in n_{1}(\alpha)$, then $\gamma=e_{r}-e_{s}$ and $\gamma+\nu \in n_{1}(\alpha)$, so that $n(\alpha)=n_{1}(\alpha)$ is as claimed.

We note that $w\left(e_{1}\right)=\Phi^{+} \backslash k\left(e_{1}\right)$. Now we already noted that the only way to express $e_{r}$ as a sum of positive roots is as $\left(e_{r}-e_{s}\right)+e_{s}$. Similarly, the only way to express $e_{1}-e_{s}$ as a sum of positive roots is as $\left(e_{1}-e_{r}\right)+\left(e_{r}-e_{s}\right)$ where $r<s$. Thus we see that $w\left(e_{1}\right)=\left\{e_{i} \mid 1 \leqslant\right.$ $i \leqslant n\} \cup\left\{e_{i}-e_{j} \mid 1 \leqslant i<j \leqslant n\right\}$ and thus $k\left(e_{1}\right)$ is as claimed.

(c) follows from the observation that $e_{r}+\left(e_{1}-e_{s}\right) \in \Phi^{+}$only if $r=s$, in which case the sum is equal to $e_{1}$.

(d) follows from the observation that the sum of any pair of roots from $a\left(e_{1}\right)$ lies in $k\left(e_{1}\right)$, which means that $a\left(e_{1}\right)$ is a pattern modulo $k\left(e_{1}\right)$. The proof of the second statement of $(\mathrm{d})$ is as the proof of Lemma $7.7(\mathrm{~d})$.

To prove part (e) we note that $s\left(e_{1}\right)$ contains all long roots of $\Phi^{+}$and the root $e_{1}$. The long roots are the positive roots of a $D_{n}$ root system and thus form a closed pattern. If $\gamma$ is a long root, then $e_{1}+\gamma \notin \Phi^{+}$. This shows (e).

To see the second part of (f) note that $\left\{e_{r}-e_{s} \mid 1<r<s \leqslant n\right\}=\Phi^{+} \backslash\left(k\left(e_{1}\right) \cup h\left(e_{1}\right)\right)$. To see the normality of the leg note that $\left(e_{1}-e_{r}\right)+\beta \in \Phi^{+}$only if $\beta \in\left\{e_{r}-e_{s}, e_{r}+e_{t} \mid r<s, r \neq t\right\}$. Hence $\left(e_{1}-e_{r}\right)+\beta=e_{1}-e_{s}$ or $e_{1}+e_{t} \in \ell\left(e_{1}\right) \cup k\left(e_{1}\right)$, which is our claim.

Now (g) follows from (f).

Finally, (e), (f), (c) and (d) are the hypotheses of Proposition 6.2 and thus (h) follows.

\subsection{Type $D$}

Let $n \geqslant 4$ be an integer. We construct a root system of type $D_{n}$ as in [23, $\left.\S 12.1\right]$. Let $e_{1}, e_{2}, \ldots, e_{n} \in \mathbb{R}^{n}$ be the usual orthonormal unit vectors which form a basis of $\mathbb{R}^{n}$. Then 
$\Phi:=\left\{ \pm\left(e_{i} \pm e_{j}\right) \mid 1 \leqslant i \neq j \leqslant n\right\}$ is a root system of type $D_{n}$ and the set $\left\{\alpha_{1}, \ldots, \alpha_{n}\right\}$, where $\alpha_{i}:=e_{i}-e_{i+1}$ for $i=1,2, \ldots, n-1$ and $\alpha_{n}:=e_{n-1}+e_{n}$, is a set of simple roots. The corresponding set of positive roots is $\Phi^{+}=\left\{e_{i} \pm e_{j} \mid 1 \leqslant i<j \leqslant n\right\}$.

From the explicit description of the root systems (or the Dynkin diagram) we see that $\left\{\alpha_{2}, \alpha_{3}, \ldots, \alpha_{n}\right\}$ generates a root subsystem $\Phi_{1^{\prime}}$ of type $D_{n-1}$, that $\left\{\alpha_{1}, \alpha_{2}, \ldots, \alpha_{n-2}, \alpha_{n}\right\}$ generates a root subsystem $\Phi_{(n-1)^{\prime}}$ of type $A_{n-1}$, and that $\left\{\alpha_{1}, \alpha_{2}, \ldots, \alpha_{n-1}\right\}$ generates a root subsystem $\Phi_{n^{\prime}}$ of type $A_{n-1}$. We set $\Phi_{1^{\prime}}^{+}:=\Phi_{1^{\prime}} \cap \Phi^{+}, \Psi_{1^{\prime}}^{+}:=\Phi^{+} \backslash \Phi_{1^{\prime}}^{+}, \Phi_{(n-1)^{\prime}}^{+}:=\Phi_{(n-1)^{\prime}} \cap \Phi^{+}$, $\Psi_{(n-1)^{\prime}}^{+}:=\Phi^{+} \backslash \Phi_{(n-1)^{\prime}}^{+}$and $\Phi_{n^{\prime}}^{+}:=\Phi_{n^{\prime}} \cap \Phi^{+}, \Psi_{n^{\prime}}^{+}:=\Phi^{+} \backslash \Phi_{n^{\prime}}^{+}$. The sets $\Psi_{1^{\prime}}^{+}, \Psi_{(n-1)^{\prime}}^{+}$and $\Psi_{n^{\prime}}^{+}$ are normal closed patterns. In fact, $P\left(\Psi_{1^{\prime}}^{+}\right), P\left(\Psi_{(n-1)^{\prime}}^{+}\right)$and $P\left(\Psi_{n^{\prime}}^{+}\right)$are the unipotent radicals of the standard parabolic subgroups corresponding to $\left\{\alpha_{2}, \alpha_{3}, \ldots, \alpha_{n}\right\},\left\{\alpha_{1}, \alpha_{2}, \ldots, \alpha_{n-2}, \alpha_{n}\right\}$ and $\left\{\alpha_{1}, \alpha_{2}, \ldots, \alpha_{n-1}\right\}$, respectively. Furthermore, the sets $\Phi_{1^{\prime}}^{+}, \Phi_{(n-1)^{\prime}}^{+}$and $\Phi_{n^{\prime}}^{+}$are closed patterns and $P\left(\Phi_{1^{\prime}}^{+}\right) \cong U D_{n} / P\left(\Psi_{1^{\prime}}^{+}\right) \cong U D_{n-1}$ and $P\left(\Phi_{n^{\prime}}^{+}\right) \cong U D_{n} / P\left(\Psi_{n^{\prime}}^{+}\right) \cong U A_{n-1}$.

Let $\alpha \in \Phi^{+}$and $\chi \in \operatorname{Irr}\left(U D_{n}\right)_{\alpha}$. Suppose that $\alpha \in \Phi_{1^{\prime}}^{+}$. It follows from Lemma 5.4 and Remark 2(a), (c) that $P\left(\Psi_{1^{\prime}}^{+}\right) \subseteq \operatorname{Ker}(\chi)$. Thus, we can identify $\chi$ with a single root character of $P\left(\Phi_{1^{\prime}}^{+}\right) \cong U D_{n} / P\left(\Psi_{1^{\prime}}^{+}\right) \cong U D_{n-1}$. In this way, the classification and construction of the elements of $\operatorname{Irr}\left(U D_{n}\right)_{\alpha}$ are reduced to the case $D_{n-1}$. Similarly, if $\alpha \in \Phi_{(n-1)^{\prime}}^{+}$or $\alpha \in \Phi_{n^{\prime}}^{+}$we can identify $\chi$ with a single root character of $P\left(\Phi_{(n-1)^{\prime}}^{+}\right)$or $P\left(\Phi_{n^{\prime}}^{+}\right)$respectively, and thereby obtain a reduction to the case $A_{n-1}$ which has already been treated in $\S 7.1$. Hence, we only have to consider positive roots $\alpha$ which are not contained in $\Phi_{1^{\prime}}^{+} \cup \Phi_{(n-1)^{\prime}}^{+} \cup \Phi_{n^{\prime}}^{+}$, that is, the roots $e_{1}+e_{i}$, where $1<i<n$. We observe that $\Phi$ is a subsystem of the root system of type $B_{n}$ described in $\S 7.2$. Thus most of what follows is a simple consequence of restricting from type $B_{n}$ to type $D_{n}$.

Lemma 7.13. Let $\Phi$ be a root system of type $D_{n}$ as described above. For all positive roots of the form $\alpha=e_{1}+e_{i}$ where $1<i<n$, we have

$$
h(\alpha)=\{\alpha\} \cup\left\{e_{1}-e_{s}, e_{s}+e_{i} \mid 1<s<i\right\} \cup\left\{e_{1} \pm e_{s}, e_{i} \mp e_{s} \mid i<s \leqslant n\right\} .
$$

Proof. This follows from Lemma 7.5 via restriction.

Next we determine the closed patterns $n(\alpha)$ and $k(\alpha)$.

Lemma 7.14. Let $\Phi$ be a root system of type $D_{n}$ as described above. For all positive roots of the form $\alpha=e_{1}+e_{i}$ where $1<i<n$ the following are true.

(a) The sets $n(\alpha)$ and $k(\alpha)$ are normal closed patterns in $\Phi^{+}$.

(b) $n(\alpha)=\left\{e_{1}+e_{s} \mid 1<s<i\right\}$.

(c) $k(\alpha)=\left\{e_{s}+e_{t} \mid 1 \leqslant s<t<i\right\}$.

Proof. This follows from Lemma 7.6 via restriction.

Let $\alpha \in \Phi^{+}$. As already mentioned above, if $\alpha \in \Phi_{(n-1)^{\prime}}^{+} \cup \Phi_{n^{\prime}}^{+}$then the hook $h(\alpha)$, the $\operatorname{arm} a(\alpha)$ and the leg $\ell(\alpha)$ are defined as for type $A_{n}$. Also if $\alpha \in \Phi_{1^{\prime}}^{+}$then we can assume recursively that $h(\alpha), a(\alpha)$ and $\ell(\alpha)$ are already defined. For $\alpha=e_{1}+e_{i}$, where $1<i<n$, we define the arm and the leg as in $\S 7.2$ via restriction:

$$
\begin{aligned}
a(\alpha) & :=\left\{e_{1}-e_{s} \mid 1<s<i\right\} \cup\left\{e_{i} \pm e_{s} \mid i<s \leqslant n\right\}, \\
\ell(\alpha) & :=\left\{e_{s}+e_{i} \mid 1<s<i\right\} \cup\left\{e_{1} \pm e_{s} \mid i<s \leqslant n\right\} .
\end{aligned}
$$

As for types $A_{n}$ and $B_{n}$, we will see that for each $\alpha \in \Phi^{+}$the hook $h(\alpha)$ is a closed pattern. We set $H_{\alpha}:=P(h(\alpha))$ and call it the hook subgroup corresponding to $\alpha$. 
Lemma 7.15. Let $\Phi$ be a root system of type $D_{n}$ as described at the beginning of this section. For all $\alpha=e_{1}+e_{i} \in \Phi^{+}$the following are true.

(a) The hook $h(\alpha)$, the arm $a(\alpha)$ and the leg $\ell(\alpha)$ are closed patterns.

(b) The pattern subgroups $P(a(\alpha))$ and $P(\ell(\alpha))$ are elementary abelian.

(c) $|a(\alpha)|=|\ell(\alpha)|=2 n-i-2$.

(d) If $\operatorname{ht}(\alpha)>1$ then the hook subgroup $H_{\alpha}$ is special of type $q^{1+2(2 n-i-2)}$ and $\left[y, H_{\alpha}\right]=$ $Z\left(H_{\alpha}\right)=X_{\alpha}$ for all $y \in H_{\alpha} \backslash Z\left(H_{\alpha}\right)$. More specifically: For each $y \in H_{\alpha} \backslash Z\left(H_{\alpha}\right)$ there is some $\beta \in h(\alpha)$ such that $\left\{\left[y, x_{\beta}(t)\right] \mid t \in \mathbb{F}_{q}\right\}=X_{\alpha}$.

Proof. This follows from Lemma 7.7 via restriction.

Lemma 7.16. Let $\Phi$ be a root system of type $D_{n}$ as described at the beginning of this section. For all $\alpha=e_{1}+e_{i} \in \Phi^{+}$the following are true.

(a) The source $s(\alpha)$ is a closed pattern.

(b) $\ell(\alpha) \cup k(\alpha) \unlhd s(\alpha)$.

(c) $\ell(\alpha) \cup\{\alpha\} \cup k(\alpha) \unlhd \Phi^{+}$.

Proof. This follows from Lemma 7.8 via restriction.

Proposition 7.17. Let $\Phi$ be a root system of type $D_{n}$ as described above. For each $\alpha=$ $e_{1}+e_{i} \in \Phi^{+}$where $1<i<n$ the map $\Psi_{\alpha}: \operatorname{Irr}\left(\bar{T}_{\alpha}\right) \times \operatorname{Irr}\left(X_{\alpha}\right)^{*} \rightarrow \operatorname{Irr}\left(U D_{n}\right)_{\alpha}$ with

$$
(\mu, \lambda) \mapsto\left(\operatorname{Infl}_{\bar{T}_{\alpha}}^{S_{\alpha}} \mu \cdot \operatorname{Infl}_{X_{\alpha}}^{S_{\alpha}} \lambda\right)^{U D_{n}}
$$

is a one-to-one correspondence with the property $\Psi_{\alpha}(\mu, \lambda)(1)=q^{2 n-i-2} \cdot \mu(1)$.

Proof. The content of Lemma 7.16 is that hypotheses (1)-(3) of Proposition 6.2 are satisfied, and the content of Lemma 7.15(d) is that hypothesis (4) of Proposition 6.2 is satisfied. This proves the correspondence.

We conclude this section by giving structural information concerning the group $\bar{T}_{\alpha}$. For all $1<i<n$ and $\alpha=e_{1}+e_{i}$ we define

$$
\begin{aligned}
d_{1, i} & :=\left\{e_{r} \pm e_{s} \mid 1<r<s \leqslant n \text { and } r \neq i \neq s\right\}, \\
\operatorname{ob}(\alpha) & :=\Phi^{+} \backslash\left(n(\alpha) \cup h(\alpha) \cup d_{1, i}\right)=\left\{e_{r}-e_{i} \mid 1 \leqslant r<i\right\}, \\
\operatorname{kob}(\alpha) & :=d_{1, i} \backslash\left\{e_{r}-e_{s} \mid 1<r<s<i\right\} .
\end{aligned}
$$

Note that $d_{1, i}$ and $\mathrm{ob}\left(e_{1}+e_{i}\right)$ are closed patterns for all $1<i<n$ and that $P(\operatorname{ob}(\alpha))$ is elementary abelian. Furthermore, we have $\operatorname{ob}(\alpha) \cap k(\alpha)=\emptyset$.

Lemma 7.18. Let $\Phi$ be a root system of type $D_{n}$ as described at the beginning of this section. For all $\alpha=e_{1}+e_{i} \in \Phi^{+}$the following are true.

(a) $S_{\alpha} / P(\ell(\alpha) \cup k(\alpha)) \cong \bar{S}_{\alpha} / P(\ell(\alpha)) P(k(\alpha)) \cong \bar{T}_{\alpha} \times X_{\alpha}$.

(b) $\bar{T}_{\alpha} \cong P\left(d_{1, i}\right) / P\left(d_{1, i} \cap k(\alpha)\right) \ltimes P(\mathrm{ob}(\alpha)) / P(\mathrm{ob}(\alpha) \cap k(\alpha))$.

(c) If $i<n$, then $\operatorname{kob}(\alpha)$ is a closed pattern that is normal in $d_{1, i}$ and $P(\operatorname{kob}(\alpha))$ centralizes $P(\mathrm{ob}(\alpha)) / P(\mathrm{ob}(\alpha) \cap k(\alpha))$.

(d) If $i=n$ then $P(\mathrm{ob}(\alpha)) / P(\mathrm{ob}(\alpha) \cap k(\alpha))=\{1\}$.

(e) If $i \in\{2,3\}$ and $i=n$ then $\bar{T}_{\alpha}=\{1\}$.

(f) If $i \in\{2,3\}$ and $i<n$ then $\operatorname{kob}(\alpha)=d_{1, i}, k(\alpha)=n(\alpha)$ and

$$
\bar{T}_{\alpha} \cong U D_{n-2} \times\left(U A_{1}\right)^{i-1} .
$$


(g) If $i>3$ then $P\left(d_{1, i}\right) / P\left(d_{1, i} \cap k(\alpha)\right)$ is isomorphic to a quotient pattern group of $U D_{n-2}$.

(h) If $i>3$ then $P\left(d_{1, i}\right) / P\left(\operatorname{kob}(\alpha) \cup\left(k(\alpha) \cap d_{1, i}\right)\right) \cong U A_{i-3}$.

Proof. This follows from Lemma 7.10 via restriction.

\subsection{Type $C$}

Let $n \geqslant 3$ be an integer. We construct a root system of type $C_{n}$ as in [23, $\left.\S 12.1\right]$. Let $e_{1}, e_{2}, \ldots, e_{n} \in \mathbb{R}^{n}$ be the usual orthonormal unit vectors which form a basis of $\mathbb{R}^{n}$. Then $\Phi:=\left\{ \pm\left(e_{i} \pm e_{j}\right) \mid 1 \leqslant i \neq j \leqslant n\right\} \cup\left\{ \pm 2 e_{i} \mid 1 \leqslant i \leqslant n\right\}$ is a root system of type $C_{n}$ and the set $\left\{\alpha_{1}, \ldots, \alpha_{n}\right\}$, where $\alpha_{i}:=e_{i}-e_{i+1}$ for $i=1,2, \ldots, n-1$ and $\alpha_{n}:=2 e_{n}$, is a set of simple roots. The corresponding set of positive roots is $\Phi^{+}=\left\{e_{i} \pm e_{j} \mid 1 \leqslant i<j \leqslant n\right\} \cup\left\{2 e_{i} \mid 1 \leqslant i \leqslant n\right\}$.

The highest long root with respect to this base is $2 e_{1}=2 \alpha_{1}+\ldots+2 \alpha_{n-1}+\alpha_{n}$, whereas the highest short root is $e_{1}+e_{2}$. We note that the long roots of $\Phi$ form a $A_{1}^{n}$-subsystem. Let $\alpha, \beta \in \Phi^{+}$with $\alpha+\beta \in \Phi^{+}$. We recall that the Chevalley commutator relations imply that if $\alpha$ is short and $\beta$ is long, then $\left[X_{\alpha}, X_{\beta}\right] \subseteq X_{\alpha+\beta} X_{2 \alpha+\beta}$, where $\alpha+\beta$ is short and $2 \alpha+\beta$ is long, and that if both $\alpha$ and $\beta$ are short, then $\left[X_{\alpha}, X_{\beta}\right] \subseteq X_{\alpha+\beta}$, where $\alpha+\beta$ is short.

From the explicit description of the root systems (or from the Dynkin diagram) we see that $\left\{\alpha_{2}, \alpha_{3}, \ldots, \alpha_{n}\right\}$ generates a root subsystem $\Phi_{1^{\prime}}$ of type $C_{n-1}$ and that $\left\{\alpha_{1}, \alpha_{2}, \ldots, \alpha_{n-1}\right\}$ generates a root subsystem $\Phi_{n^{\prime}}$ of type $A_{n-1}$. We set $\Phi_{1^{\prime}}^{+}:=\Phi_{1^{\prime}} \cap \Phi^{+}, \Psi_{1^{\prime}}^{+}:=\Phi^{+} \backslash \Phi_{1^{\prime}}^{+}$ and $\Phi_{n^{\prime}}^{+}:=\Phi_{n^{\prime}} \cap \Phi^{+}, \Psi_{n^{\prime}}^{+}:=\Phi^{+} \backslash \Phi_{n^{\prime}}^{+}$. The sets $\Psi_{1^{\prime}}^{+}$and $\Psi_{n^{\prime}}^{+}$are normal closed patterns. In fact, $P\left(\Psi_{1^{\prime}}^{+}\right)$and $P\left(\Psi_{n^{\prime}}^{+}\right)$are the unipotent radicals of the standard parabolic subgroups corresponding to $\left\{\alpha_{2}, \alpha_{3}, \ldots, \alpha_{n}\right\}$ and $\left\{\alpha_{1}, \alpha_{2}, \ldots, \alpha_{n-1}\right\}$, respectively. Furthermore, the sets $\Phi_{1^{\prime}}^{+}$and $\Phi_{n^{\prime}}^{+}$are closed patterns and $P\left(\Phi_{1^{\prime}}^{+}\right) \cong U C_{n} / P\left(\Psi_{1^{\prime}}^{+}\right) \cong U C_{n-1}$ and $P\left(\Phi_{n^{\prime}}^{+}\right) \cong$ $U C_{n} / P\left(\Psi_{n^{\prime}}^{+}\right) \cong U A_{n-1}$.

Let $\alpha \in \Phi^{+}$and $\chi \in \operatorname{Irr}\left(U C_{n}\right)_{\alpha}$. Suppose that $\alpha \in \Phi_{1^{\prime}}^{+}$. It follows from Lemma 5.4 and Remark 2(a), (c) that $P\left(\Psi_{1^{\prime}}^{+}\right) \subseteq \operatorname{Ker}(\chi)$. Thus, we can identify $\chi$ with a single root center character of $P\left(\Phi_{1^{\prime}}^{+}\right) \cong U C_{n} / P\left(\Psi_{1^{\prime}}^{+}\right) \cong U C_{n-1}$. In this way, the classification and construction of the elements of $\operatorname{Irr}\left(U C_{n}\right)_{\alpha}$ are reduced to the case $C_{n-1}$. Similarly, if $\alpha \in \Phi_{n^{\prime}}^{+}$we can identify $\chi$ with a single root center character of $P\left(\Phi_{n^{\prime}}^{+}\right)$and thereby get a reduction to the case $A_{n-1}$ which has already been treated in $\S 7.1$. Hence, we only have to consider positive roots $\alpha$ which are not contained in $\Phi_{1^{\prime}}^{+} \cup \Phi_{n^{\prime}}^{+}$, that is, the roots $e_{1}+e_{i}$ where $1<i \leqslant n$ and the root $2 e_{1}$. We begin with single root characters lying above the highest root $2 e_{1}$.

Lemma 7.19. Let $\Phi$ be a root system of type $C_{n}$ as described above. For $\alpha=2 e_{1}$ we have

$$
h(\alpha)=\left\{2 e_{1}, e_{1}+e_{s}, e_{1}-e_{s} \mid 2 \leqslant s \leqslant n\right\} .
$$

Proof. We observe that the sum of two long roots is never in $\Phi^{+}$. Next we observe that the sum of a long and a short root is either short or not in $\Phi^{+}$. Thus if $2 e_{1}=\beta+\gamma$, then both $\beta$ and $\gamma$ are short. This implies that $\beta=e_{1} \pm e_{s}$ and hence $\gamma=e_{1} \mp e_{s}$. This proves our claim.

We define the arm $a\left(2 e_{1}\right)$ and the leg $\ell\left(2 e_{1}\right)$ of $h\left(2 e_{1}\right)$ as follows:

$$
a\left(2 e_{1}\right):=\left\{e_{1}-e_{s} \mid 1<s \leqslant n\right\} \quad \text { and } \quad \ell\left(2 e_{1}\right):=\left\{e_{1}+e_{s} \mid 1<s \leqslant n\right\} .
$$

Lemma 7.20. The following are true.

(a) $n\left(2 e_{1}\right)=k\left(2 e_{1}\right)=\emptyset$.

(b) $\ell(\alpha) \cup\{\alpha\} \unlhd h(\alpha)$.

(c) The group $P\left(h\left(2 e_{1}\right)\right)$ is special of order $q^{1+2(n-1)}$. Moreover, $\left[y, H_{2 e_{1}}\right]=Z\left(H_{2 e_{1}}\right)=X_{2 e_{1}}$ for all $y \in H_{2 e_{1}} \backslash Z\left(H_{2 e_{1}}\right)$. 
(d) $s\left(2 e_{1}\right)$ is a closed pattern.

(e) $\ell\left(2 e_{1}\right) \unlhd s\left(2 e_{1}\right)$ and $P\left(\ell\left(2 e_{1}\right)\right)$ is elementary abelian.

(f) $s\left(2 e_{1}\right) \backslash\left(\left\{2 e_{1}\right\} \cup \ell\left(2 e_{1}\right)\right)$ is a closed pattern of type $U C_{n-1}, \bar{T}_{2 e_{1}} \cong U C_{n-1}$, and $S_{2 e_{1}} / P\left(\ell\left(2 e_{1}\right)\right) \cong \bar{T}_{2 e_{1}} \times X_{2 e_{1}}$.

Proof. As $2 e_{1}$ is the highest root of $\Phi^{+}$, part (a) is clear. Also we note that $2 e_{1}$ is the unique long root in $h\left(2 e_{1}\right)$. The short roots in $h(\alpha)$ are all of the form $e_{1} \pm e_{r}$ with $r>1$ and thus the sum of two of these lies in $\Phi^{+}$if and only if that sum is $2 e_{1}$. This combined with Lemma 3.3 proves part (b) and the first half of part (c). The proof of the Lemma 7.2(d) carries over verbatim to prove the second part of (c).

Now let $\beta:=e_{1}-e_{s} \in a\left(2 e_{1}\right)$ and suppose that $\beta=\gamma+\gamma^{\prime}$ with $\gamma, \gamma^{\prime} \in \Phi^{+}$. As $\beta \in \Phi_{n^{\prime}}$, we see that without loss $\gamma=e_{1}-e_{r}$ and $\gamma^{\prime}=e_{r}-e_{s}$ with $1<r<s$. As $\gamma \in a\left(2 e_{1}\right)$ this proves part (d). Now if $e_{1}+e_{r}, e_{1}+e_{s} \in \ell\left(2 e_{1}\right)$, then $e_{1}+e_{r}+e_{1}+e_{s}=2 e_{1}+e_{r}+e_{s} \notin \Phi^{+}$, and thus the Chevalley commutator relations imply that $P\left(\ell\left(2 e_{1}\right)\right)$ is elementary abelian. If $e_{1}+e_{s} \in \ell\left(2 e_{1}\right)$ and $\beta \in s\left(2 e_{1}\right)$, then $e_{1}+e_{s}+\beta \in \Phi^{+}$only if $\beta=e_{r}-e_{s}$ where $r<s$ and then $e_{1}+e_{s}+\beta \in \ell\left(2 e_{1}\right)$ proving (e).

Finally, we observe that

$$
s\left(2 e_{1}\right) \backslash\left(\left\{2 e_{1}\right\} \cup \ell\left(2 e_{1}\right)\right)=\left\{2 e_{i} \mid 2 \leqslant i \leqslant n\right\} \cup\left\{e_{r} \pm e_{s} \mid 2 \leqslant r<s \leqslant n\right\}
$$

from which the claims in (f) follow.

Proposition 7.21. Let $\Phi$ be a root system of type $C_{n}$ as described above. For the root $\alpha:=2 e_{1} \in \Phi^{+}$the map $\Psi_{\alpha}: \operatorname{Irr}\left(\bar{T}_{\alpha}\right) \times \operatorname{Irr}\left(X_{\alpha}\right)^{*} \rightarrow \operatorname{Irr}\left(U C_{n}\right)_{\alpha}$ with

$$
(\mu, \lambda) \mapsto\left(\operatorname{Infl}_{\bar{T}_{\alpha}}^{S_{\alpha}} \mu \cdot \operatorname{Infl}_{X_{\alpha}}^{S_{\alpha}} \lambda\right)^{U C_{n}}
$$

is a one-to-one correspondence with $\Psi_{\alpha}(\mu, \lambda)(1)=q^{n-1} \cdot \mu(1)$, where $\bar{T}_{\alpha} \cong U C_{n-1}$.

Proof. The content of Lemma 7.20(d), (e), (b) and (c) is that hypotheses (1)-(4) of Proposition 6.2 are satisfied. This proves the correspondence. The statement about the structure of $\bar{T}_{\alpha}$ is the content of Lemma $7.20(\mathrm{f})$.

Next we consider the short roots $\alpha=e_{1}+e_{i}$ which are not contained in $\Phi_{1^{\prime}}^{+} \cup \Phi_{n^{\prime}}^{+}$.

Lemma 7.22. Let $\Phi$ be a root system of type $C_{n}$ as described above. For all positive roots of the form $\alpha=e_{1}+e_{i}$ we have

$$
h(\alpha)=\{\alpha\} \cup\left\{e_{1}-e_{s}, e_{s}+e_{i} \mid 1<s<i\right\} \cup\left\{e_{1} \pm e_{s}, e_{i} \mp e_{s} \mid i<s \leqslant n\right\} \cup\left\{2 e_{i}, e_{1}-e_{i}\right\} .
$$

Proof. Let $\beta=2 e_{t}, \beta^{\prime}=e_{s^{\prime}} \pm e_{t^{\prime}} \in \Phi^{+}$with $\beta+\beta^{\prime}=\alpha$. Then $\beta^{\prime}=e_{1}-e_{i}$ and $\beta=2 e_{i}$.

Next, let $\beta=e_{s} \pm e_{t}, \beta^{\prime}=e_{s^{\prime}} \pm e_{t^{\prime}} \in \Phi^{+}$such that $\beta+\beta^{\prime}=\alpha$. We see immediately that one of the two \pm signs has to be a + sign, that the other has to be a - sign and that $t=t^{\prime}$. Furthermore, we can assume $s=1$ and $s^{\prime}=i$. Hence

$$
\left(\beta, \beta^{\prime}\right) \in\left\{\left(e_{1}-e_{s}, e_{s}+e_{i}\right) \mid 1<s<i \text { or } i<s \leqslant n\right\} \cup\left\{\left(e_{1}+e_{s}, e_{i}-e_{s}\right) \mid i<s \leqslant n\right\}
$$

and the claim follows.

The content of Remark 2(c) and Lemma 5.4 is that if $\chi \in \operatorname{Irr}(X U)_{\alpha}$, then $\operatorname{rk}(\chi)=k(\alpha)$. Also recall that Lemma 5.6 states $w(\alpha) \cap k(\alpha)=\emptyset$ for each $\alpha \in \Phi^{+}$. Using these two facts, we can now describe the closed patterns $n(\alpha)$ and $k(\alpha)$. 
Lemma 7.23. Let $\Phi$ be a root system of type $C_{n}$ as described above and let $\alpha=e_{1}+e_{i}$. The following are true.

(a) The sets $n(\alpha)$ and $k(\alpha)$ are normal closed patterns in $\Phi^{+}$.

(b) $n(\alpha)=\left\{e_{1}+e_{s} \mid 1<s<i\right\} \cup\left\{2 e_{1}\right\}$.

(c) $k(\alpha)=n(\alpha) \cup\left\{e_{r}+e_{s} \mid 1<r<s<i\right\} \cup\left\{2 e_{r} \mid 1<r<i\right\}$.

Proof. Part (a) was established in $\S 5$.

(b) By definition $n_{0}(\alpha)=\left\{e_{1}+e_{i}\right\}$. Let $\gamma=e_{k} \pm e_{l} \in \Phi^{+}$. Then $\alpha+\gamma \in \Phi^{+}$if and only if $\gamma=e_{s}-e_{i}$ for some $1<s<i$ or $\gamma=e_{1}-e_{i}$. In the first case we have $\alpha+\gamma=e_{1}+e_{s}$ where $1<s<i$, and in the second case $\alpha+\gamma=2 e_{1}$. Now if $\gamma^{\prime}=2 e_{r}$, then $\gamma^{\prime}+\delta \notin \Phi^{+}$for all $\delta=e_{r}+e_{t}$. Thus, $n_{1}(\alpha)=\left\{e_{1}+e_{s} \mid 1<s<i\right\} \cup\left\{2 e_{1}\right\}$. Again, if $\gamma=e_{k} \pm e_{l} \in \Phi^{+}$then $\gamma+\left(e_{1}+e_{s}\right) \in \Phi^{+}$if and only if $\gamma=e_{t}-e_{s}$ for some $1<t<s$, and in this case we have $\gamma+\left(e_{1}+e_{s}\right)=e_{1}+e_{t}$. Thus $n_{2}(\alpha)=n_{1}(\alpha)$ and therefore $n(\alpha)=n_{1}(\alpha)$ and (b) follows.

(c) Let $\beta=e_{s}+e_{t} \in \Phi^{+}$where $s<t$ and $t \geqslant i$. If $s=1$ then $\beta \in h(\alpha) \subseteq w(\alpha)$. If $s>1$ then $\beta \in h\left(e_{1}+e_{t}\right)$ and $e_{1}+e_{t} \in h(\alpha)$, hence $\beta \in w(\alpha)$.

Let $\beta=2 e_{s}$ with $i<s$. Then $\beta+\left(e_{1}-e_{s}\right)=e_{1}+e_{s} \in h(\alpha) \subseteq w(\alpha)$.

Now let $\beta=e_{s}-e_{t} \in \Phi^{+}$where $s<t$. If $s=1$ then $\beta \in h(\alpha) \subseteq w(\alpha)$. If $s>1$ and $t \neq i$ then $\beta \in h\left(e_{1}-e_{t}\right)$ and $e_{1}-e_{t} \in h(\alpha)$. Hence $\beta \in w(\alpha)$. If $s>1$ and $t=i$ then $\beta \in h\left(e_{1}-e_{i}\right), e_{1}-e_{i} \in h(\alpha)$ and so we have $\beta \in w(\alpha)$. It follows from Lemma 5.6 that $k(\alpha) \subseteq\left\{e_{s}+e_{t} \mid 1 \leqslant s<t<i\right\} \cup\left\{2 e_{s} \mid s<i\right\}=: M$.

We claim that $M \unlhd \Phi^{+}$. Let $\beta=e_{s}+e_{t} \in M, \gamma \in \Phi^{+}$with $\beta+\gamma \in \Phi^{+}$. It follows that $\gamma$ is of the form $\gamma=e_{k}-e_{s}$ with $k<s<t<i$, or $\gamma=e_{k}-e_{t}$ with $k<t<i$. In the first case we have $\beta+\gamma=e_{k}+e_{t}$ with $k<t<i$, in the second case we have $\beta+\gamma=e_{k}+e_{s}$ with $k, s<i$. Thus, $\beta+\gamma \in M$ in both cases. Let $\beta=2 e_{s} \in M, \gamma \in \Phi^{+}$with $\beta+\gamma \in \Phi^{+}$. It follows that $\gamma$ is of the form $\gamma=e_{k}-e_{s}$ with $k<s<i$ and that $\beta+\gamma=e_{k}+e_{s}$ with $k<s<i$, hence $\beta+\gamma \in M$.

Thus we have $M \unlhd \Phi^{+}$. By the definition of $M$ we have $\alpha \notin M$. Hence Remark 2(a) implies that $M \subseteq k(\alpha)$. Thus we have $k(\alpha)=M$ and (c) follows. This completes the proof.

We define

$$
\ell\left(e_{1}+e_{i}\right):=\left\{e_{1} \pm e_{s} \mid i<s \leqslant n\right\} \cup\left\{e_{1}-e_{i}\right\} \cup\left\{e_{s}+e_{i} \mid 1<s<i\right\}
$$

and

$$
a\left(e_{1}+e_{i}\right):=\left\{e_{i} \mp e_{s} \mid i<s \leqslant n\right\} \cup\left\{2 e_{i}\right\} \cup\left\{e_{1}-e_{s} \mid 1<s<i\right\} .
$$

As before, we will show that for each $\alpha \in \Phi^{+}$the hook $h(\alpha)$ is a closed pattern modulo $k(\alpha)$. Call $H_{\alpha}:=P(h(\alpha) \cup k(\alpha)) / P(k(\alpha))$ the hook subgroup corresponding to $\alpha$, always bearing in mind that we calculate modulo $P(k(\alpha))$.

Lemma 7.24. Let $\Phi$ be a root system of type $C_{n}$ as described at the beginning of this subsection. For all $\alpha=e_{1}+e_{i} \in \Phi^{+}$the following are true.

(a) The hook $h(\alpha) \cup k(\alpha)$ and the leg $\ell(\alpha) \cup k(\alpha)$ are closed patterns.

(b) $\{\alpha\} \cup \ell(\alpha) \cup k(\alpha) \unlhd h(\alpha) \cup k(\alpha)$.

(c) $P(a(\alpha))$ is a pattern subgroup. It is isomorphic to a product of an elementary abelian group of order $q^{i-2}$ and a special group of order $q^{1+2(n-i)}$. The center of the special group is $X_{2 e_{i}}$.

(d) $|a(\alpha)|=2 n-i-1$.

(e) If $\operatorname{ht}(\alpha)>1$, then for each $y \in\left(\prod_{\gamma \in a(\alpha)} X_{\gamma}\right) \backslash\{1\}$ there is some $\beta \in \ell(\alpha)$ such that $\left\{\left[y, x_{\beta}(t)\right] \mid t \in \mathbb{F}_{q}\right\}=X_{\alpha}$. 
Proof. (a), (b) Let $\beta, \gamma \in h(\alpha)$. We have $\beta+\gamma \in \Phi^{+}$if and only if $\{\beta, \gamma\}=\left\{e_{1}-e_{s}, e_{s}+e_{i}\right\}$ for some $s \neq i$, or $\{\beta, \gamma\}=\left\{e_{1}-e_{i}, e_{s}+e_{i}\right\}$ for some $s \neq i$, or $\{\beta, \gamma\}=\left\{e_{1}+e_{s}, e_{i}-e_{s}\right\}$ for some $s>i$, or $\{\beta, \gamma\}=\left\{e_{i}+e_{s}, e_{i}-e_{s}\right\}$ for some $s>i$, or $\{\beta, \gamma\}=\left\{e_{1}-e_{i}, e_{i}-e_{s}\right\}$ for some $s>i$, or $\{\beta, \gamma\}=\left\{e_{1}+e_{s}, e_{1}-e_{s}\right\}$ for some $s>i$, or $\{\beta, \gamma\}=\left\{2 e_{i}, e_{1}-e_{i}\right\}$. In all of these cases $\beta+\gamma \in h(\alpha) \cup k(\alpha)$, and it follows that $h(\alpha) \cup k(\alpha)$ is a closed pattern. We also see that for all $\beta, \gamma \in \ell(\alpha)$ with $\beta+\gamma \in \Phi^{+}$we have $\beta+\gamma \in k(\alpha)$. Hence $\ell(\alpha) \cup k(\alpha)$ is a closed pattern and (a) follows.

If $\beta \in h(\alpha)$ and $\gamma \in \ell(\alpha)$, then either $\beta+\gamma \in\left\{\alpha, 2 e_{1}\right\}$ or $\beta+\gamma \notin \Phi^{+}$, and (b) follows.

Also for $\beta, \gamma \in a(\alpha)$ we see that $\beta+\gamma \in\left\{2 e_{i}\right\}$ if $\beta, \gamma \in a(\alpha) \backslash\left\{e_{1}-e_{s} \mid s<i\right\}$ and that $\beta, \gamma \notin \Phi^{+}$if $\beta, \gamma \in\left\{e_{1}-e_{s} \mid s<i\right\}$. This shows (c).

(d) follows from (c).

Finally, suppose that $\operatorname{ht}(\alpha)>1$. We have seen in the proof of (a) and (b) that for all $\beta$, $\gamma \in h(\alpha)$ we have $\beta+\gamma \in \Phi^{+}$if and only if $\{\beta, \gamma\}=\left\{e_{1}-e_{s}, e_{s}+e_{i}\right\}$ for some $s \neq i$, or $\{\beta, \gamma\}=\left\{e_{1}-e_{i}, e_{s}+e_{i}\right\}$ for some $s \neq i$, or $\{\beta, \gamma\}=\left\{e_{1}+e_{s}, e_{i}-e_{s}\right\}$ for some $s>i$, or $\{\beta, \gamma\}=\left\{e_{i}+e_{s}, e_{i}-e_{s}\right\}$ for some $s>i$, or $\{\beta, \gamma\}=\left\{e_{1}-e_{i}, e_{i}-e_{s}\right\}$ for some $s>i$, or $\{\beta, \gamma\}=\left\{e_{1}+e_{s}, e_{1}-e_{s}\right\}$ for some $s>i$, or $\{\beta, \gamma\}=\left\{2 e_{i}, e_{1}-e_{i}\right\}$. In all of these cases $\beta+\gamma \in\{\alpha\} \cup\left\{e_{1} \pm e_{s} \mid i<s \leqslant n\right\} \cup\left\{2 e_{i}\right\} \cup k(\alpha)$. It follows that $Z\left(H_{\alpha}\right)=X_{\alpha}$ and that $P(\{\alpha\} \cup \ell(\alpha) \cup k(\alpha)) / P(k(\alpha))$ is normal and elementary abelian. Now let $y=\prod_{\gamma \in h(\alpha)} x_{\gamma}\left(t_{\gamma}\right) \in\left(\prod_{\gamma \in a(\alpha)} X_{\gamma}\right) \backslash\{1\}$. Because $y \neq 1$ there is some $\gamma \in a(\alpha) \backslash\{\alpha\}$ such that $t_{\gamma} \neq 0$. If $\gamma \neq 2 e_{i}$ then we pick $\beta:=\alpha-\gamma \in h(\alpha)$ and we get from Lemma 3.1(a) that $\left\{\left[y, x_{\beta}(t)\right] \mid t \in \mathbb{F}_{q}\right\}=X_{\alpha}=Z\left(H_{\alpha}\right)$. If $y \in X_{2 e_{i}} P(\{\alpha\} \cup \ell(\alpha) \cup k(\alpha)) \backslash P(\{\alpha\} \cup \ell(\alpha) \cup k(\alpha))$, then we pick $\beta:=e_{1}-e_{i}$ and again we get that $\left\{\left[y, x_{\beta}(t)\right] \mid t \in \mathbb{F}_{q}\right\}=X_{\alpha}=Z\left(H_{\alpha}\right)$.

Next we show the following lemma.

Lemma 7.25. Let $\Phi$ be a root system of type $C_{n}$ as described at the beginning of this subsection. For all $\alpha=e_{1}+e_{i} \in \Phi^{+}$the following are true.

(a) The source $s(\alpha)$ is a closed pattern and $k(\alpha) \subseteq s(\alpha)$.

(b) $\ell(\alpha) \cup k(\alpha) \unlhd s(\alpha)$.

Proof. (a) By Lemma 5.6 we have $k(\alpha) \cap a(\alpha) \subseteq k(\alpha) \cap w(\alpha)=\emptyset$. Hence $k(\alpha) \subseteq \Phi^{+} \backslash a(\alpha)=$ $s(\alpha)$. Let $\beta \in a(\alpha)$ and $\gamma, \gamma^{\prime} \in \Phi^{+}$such that $\beta=\gamma+\gamma^{\prime}$.

To prove that $s(\alpha)$ is a pattern it suffices to show that $\gamma \in a(\alpha)$ or $\gamma^{\prime} \in a(\alpha)$. Suppose that $\beta=e_{1}-e_{s}$ where $1<s<i$. Then $\left\{\gamma, \gamma^{\prime}\right\}=\left\{e_{1}-e_{l}, e_{l}-e_{s}\right\}$ for some $1<l<s<i$ and hence $\gamma \in a(\alpha)$ or $\gamma^{\prime} \in a(\alpha)$. Now suppose that $\beta=e_{i}+e_{s}$ where $i<s \leqslant n$. Then $\left\{\gamma, \gamma^{\prime}\right\}=\left\{e_{i}+e_{l}, e_{s}-e_{l}\right\}$ for some $i<l \leqslant n$ or $\left\{\gamma, \gamma^{\prime}\right\}=\left\{e_{s}+e_{l}, e_{i}-e_{l}\right\}$ for some $i<l \leqslant n$. Hence in both cases $\gamma \in a(\alpha)$ or $\gamma^{\prime} \in a(\alpha)$. Next, suppose that $\beta=e_{i}-e_{s}$ where $i<s \leqslant n$. Then $\left\{\gamma, \gamma^{\prime}\right\}=\left\{e_{i}-e_{l}, e_{l}-e_{s}\right\}$ for some $i<l<s \leqslant n$ and again $\gamma \in a(\alpha)$ or $\gamma^{\prime} \in a(\alpha)$. Finally, suppose that $\beta=2 e_{i}$, then $\left\{\gamma, \gamma^{\prime}\right\}=\left\{e_{i}+e_{s}, e_{i}-e_{s}\right\}$ for some $i<s \leqslant n$. But then we have $\gamma, \gamma^{\prime} \in a(\alpha)$. This proves (a).

(b) Let $\beta \in \ell(\alpha), \gamma \in s(\alpha)$. First suppose that $\beta=e_{1}+e_{s}$ where $i<s \leqslant n$. Then $\beta+\gamma \in \Phi^{+}$ only if $\gamma=e_{r}-e_{s}$ with $i \neq r<s$ (as $\left.e_{i}-e_{s} \in a(\alpha)\right)$ and thus $\beta+\gamma=e_{1}+e_{r} \in k(\alpha) \cup \ell(\alpha)$.

Next suppose that $\beta=e_{1}-e_{s}$ where $i<s \leqslant n$. Then $\beta+\gamma \in \Phi^{+}$only if $\gamma=e_{r}+e_{s}$ with $i \neq r\left(\right.$ as $\left.e_{i}+e_{s} \in a(\alpha)\right)$ or $\gamma=e_{s}-e_{r}$ with $s<r$. Thus $\beta+\gamma=e_{1}+e_{r} \in k(\alpha) \cup \ell(\alpha)$ or $\beta+\gamma=e_{1}-e_{r} \in \ell(\alpha)$.

Next suppose that $\beta=e_{s}+e_{i}$ where $s<i$. Then $\beta+\gamma \in \Phi^{+}$only if $\gamma=e_{r}-e_{s}$ with $r<s$ or $\gamma=e_{r}-e_{i}$ with $r<i$. Thus $\beta+\gamma \in\left\{e_{r}+e_{s} \mid r<s<i\right\} \cup\left\{e_{r}+e_{i} \mid r<i\right\} \subseteq k(\alpha) \cup \ell(\alpha)$.

Finally, suppose that $\beta=e_{1}-e_{i}$. Then $\beta+\gamma \in \Phi^{+}$only if $\gamma=e_{1}+e_{i}$ or $e_{i}+e_{r}$ with $r<i$ or $e_{i}-e_{r}$ with $r>i$. As $\gamma \in s(\alpha)$ we see that $\gamma=e_{1}+e_{i}$ or $e_{i}+e_{r}$ with $r<i$. Thus $\beta+\gamma \in\left\{2 e_{1}\right\} \cup\left\{e_{1}+e_{r} \mid r<i\right\} \cup\left\{e_{1}-e_{r} \mid r>i\right\} \subseteq k(\alpha) \cup \ell(\alpha)$. Now (b) follows. 
Proposition 7.26. Let $\Phi$ be a root system of type $C_{n}$ as described above. For each $\alpha=$ $e_{1}+e_{i} \in \Phi^{+}$where $1<i \leqslant n$ the map $\Psi_{\alpha}: \operatorname{Irr}\left(\bar{T}_{\alpha}\right) \times \operatorname{Irr}\left(X_{\alpha}\right)^{*} \rightarrow \operatorname{Irr}\left(U C_{n}\right)_{\alpha}$ with

$$
(\mu, \lambda) \mapsto\left(\operatorname{Inf}_{\bar{T}_{\alpha}}^{S_{\alpha}} \mu \cdot \operatorname{Infl}_{X_{\alpha}}^{S_{\alpha}} \lambda\right)^{U A_{n}}
$$

is a one-to-one correspondence with the property $\Psi_{\alpha}(\mu, \lambda)(1)=q^{2 n-i-1} \cdot \mu(1)$.

Proof. The content of Lemma 7.25(a), (b) is that hypotheses (1) and (2) of Proposition 6.2 are satisfied, and the content of Lemma 7.24(b) and (e) is that hypotheses (3) and (4) of Proposition 6.2 are satisfied. This proves the correspondence.

We now derive some structural information about $\bar{T}_{\alpha}$. To this end we define closed patterns

$$
c_{i, j}:=\left\{2 e_{t}, e_{r} \pm e_{s} \mid r, s, t \notin\{i, j\}, \text { and } r<s\right\}
$$

where $1 \leqslant i<j \leqslant n$ and

$$
\mathrm{ob}\left(e_{1}+e_{i}\right):=\left\{e_{2}-e_{i}, \ldots, e_{i-1}-e_{i}\right\} \quad \text { and } \quad \mathrm{kob}=c_{1, i} \backslash\left\{e_{r}-e_{s} \mid 1<r<s<i\right\} .
$$

Also we define $\bar{T}_{\alpha}:=S_{\alpha} / P(\{\alpha\} \cup \ell(\alpha) \cup k(\alpha))$.

Lemma 7.27. If $\alpha=e_{1}+e_{i}$, then $S_{\alpha} / P(\ell(\alpha) \cup k(\alpha)) \cong \bar{S}_{\alpha} / P(\ell(\alpha)) \cong \bar{T}_{\alpha} \times X_{\alpha}$ and $\bar{T}_{\alpha}$ is a semidirect product of $P(\mathrm{ob}(\alpha))$ with $P\left(c_{1, i}\right) / P\left(c_{1, i} \cap k(\alpha)\right)$, where $c_{1, i}$ is as above. Moreover, the following are true.

(a) The kernel of the action of $P\left(c_{1, i}\right)$ on $P(\mathrm{ob})$ is $P(\mathrm{kob})$.

(b) For $i>3, P\left(c_{1, i} /\left(c_{1, i} \cap k(\alpha)\right)\right) \cong U C_{n-2} / Z_{i-2}\left(U C_{n-2}\right)$,

(c) For $i>3, P\left(c_{1, i}\right) / P(\mathrm{kob}) \cong U A_{i-3}$,

(d) If $i=2$, then $\mathrm{kob}=c_{1,2}, \mathrm{ob}=\emptyset, k(\alpha)=n(\alpha)=\left\{2 e_{1}\right\}$, and $\bar{T}_{\alpha} \cong U C_{n-2}$.

(e) If $i=3$, then kob $=c_{1,3}, k(\alpha)=\left\{2 e_{1}, 2 e_{2}, e_{1}+e_{2}\right\}$, and $\bar{T}_{\alpha} \cong U C_{n-2} / Z\left(U C_{n-2}\right) \times$ $X_{e_{2}-e_{3}}$.

Proof. The fact that $\bar{S}_{\alpha} / P(\ell(\alpha)) \cong \bar{T}_{\alpha} \times X_{\alpha}$ follows from the definition of $\bar{T}_{\alpha}$ and parts (a) and (b) of Lemma 7.25.

(a) The set $\operatorname{ob}(\alpha)$ is the set of roots of $\Phi^{+}$which are not contained in $k(\alpha) \cup h(\alpha) \cup c_{1, i}$. Notice that $\mathrm{ob}(\alpha)$ is normalized by $c_{1, i}$, proving the first part of (a).

For the second part we observe that no root of $c_{1, i}$ has $e_{i}$ in its support. Thus the only roots from $c_{1, i}$ that can be added to an element of ob $(\alpha)$ to yield an element of $\Phi^{+}$are of the form $e_{r}-e_{s}$ where $1<r<s<i$. This yields the second part of (a).

(b) We note that $c_{1, i} \subseteq \Phi_{1^{\prime}}^{+}$and so our claim follows from part (c) of Lemma 7.23.

(c) We observe that $r, s \neq 1, i$; then $\left(e_{r}-e_{s}\right)+\left(e_{k}-e_{i}\right) \in \Phi^{+}$if and only if $1<r<s=k$. Next we note that $\left(e_{r}+e_{s}\right)+\left(e_{k}-e_{i}\right) \notin \Phi^{+}$for all $r, s \neq 1, i$ and that $e_{r}+\left(e_{k}-e_{i}\right) \notin \Phi^{+}$for all $r \neq 1, i$. Thus (c) follows.

(d) and (e) follow from (a) and Lemma 7.23.

We can now complete the proof of Theorem 1.1.

Proof of Theorem 1.1. The theorem follows from Propositions 7.4, 7.9, Lemma 7.12(h) and Propositions 7.17, 7.21 and 7.26.

\section{Single root midafis of exceptional groups}

In this section we deal with the case that the root system $\Phi$ is irreducible of type $E_{6}, E_{7}$, $E_{8}, F_{4}$ or $G_{2}$ and prove Theorems 1.2 and 1.3. We will use the explicit construction of these root systems given in $[\mathbf{2 3}, \S 12.1]$. We assume the setting and notation from $\S \S 2-6$. We assume throughout this section that Hypothesis 1 holds. 


\subsection{Types $E_{6}, E_{7}, E_{8}$}

We deal with types $E_{6}$ and $E_{7}$ by considering suitable root subsystems of a root system of type $E_{8}$. The root system of type $E_{8}$ is constructed as follows (see $[\mathbf{2 3}, \S 12.1]$ ). Let $e_{1}, e_{2}, \ldots, e_{8} \in \mathbb{R}^{8}$ be the usual orthonormal unit vectors which form a basis of $\mathbb{R}^{8}$ and let $\Phi_{8}$ be the union of the sets $\left\{ \pm\left(e_{i} \pm e_{j}\right) \mid 1 \leqslant i \neq j \leqslant 8\right\}$ and

$$
\left\{\frac{1}{2}\left( \pm e_{1} \pm e_{2} \pm e_{3} \pm e_{4} \pm e_{5} \pm e_{6} \pm e_{7} \pm e_{8}\right) \mid \text { the number of minus signs is even }\right\} \text {. }
$$

Then $\Phi_{8}$ is a root system of type $E_{8}$ and the set $\left\{\alpha_{1}, \ldots, \alpha_{8}\right\}$, where

$$
\begin{aligned}
& \alpha_{1}:=\frac{1}{2}\left(e_{1}+e_{8}-e_{2}-e_{3}-e_{4}-e_{5}-e_{6}-e_{7}\right), \quad \alpha_{2}:=e_{1}+e_{2}, \quad \alpha_{3}:=e_{2}-e_{1}, \\
& \alpha_{4}:=e_{3}-e_{2}, \quad \alpha_{5}:=e_{4}-e_{3}, \quad \alpha_{6}:=e_{5}-e_{4}, \quad \alpha_{7}:=e_{6}-e_{5}, \quad \alpha_{8}:=e_{7}-e_{6}
\end{aligned}
$$

is a set of simple roots. The corresponding set of positive roots is

$$
\Phi_{8}^{+}=\left\{e_{i} \pm e_{j} \mid 1 \leqslant j<i \leqslant 8\right\} \cup\left\{\frac{1}{2}\left( \pm e_{1} \pm e_{2} \pm e_{3} \pm e_{4} \pm e_{5} \pm e_{6} \pm e_{7}+e_{8}\right)\right\},
$$

where the number of minus signs of the coefficients of the vectors in the second set is even.

The subsystem of $\Phi_{8}$ generated by $\left\{\alpha_{1}, \ldots, \alpha_{6}\right\}$ is a root system of type $E_{6}$ which we denote by $\Phi_{6}$, whereas the subsystem generated by $\left\{\alpha_{1}, \ldots, \alpha_{7}\right\}$ is a root system of type $E_{7}$ which we denote by $\Phi_{7}$. For $i \in\{6,7\}$, the set $\left\{\alpha_{1}, \ldots, \alpha_{i}\right\}$ is a set of simple roots for $\Phi_{i}$ and $\Phi_{i}^{+}:=\Phi_{8}^{+} \cap \Phi_{i}$ is the corresponding set of positive roots. For $\alpha \in \Phi_{i}^{+}$we always take $k(\alpha)$ with respect to $\Phi_{8}$ in this section.

We number the positive roots of $\Phi_{8}$ according to Table A.1. This table contains the following information. The first column fixes the notation for the positive roots of $\Phi_{8}$. The second column lists the coefficients $m_{j}$ when the root $\alpha_{i}=\sum_{j=1}^{8} m_{j} \alpha_{j}$ is written as a linear combination of the simple roots $\alpha_{1}, \ldots, \alpha_{8}$. The third column expresses the root $\alpha_{i}$ as a linear combination of the vectors $e_{1}, \ldots, e_{8}$ and the last column contains the height $h t\left(\alpha_{i}\right)$. For example, the positive root $\alpha_{69}$ is

$$
\begin{aligned}
\alpha_{69} & =1 \cdot \alpha_{1}+2 \cdot \alpha_{2}+2 \cdot \alpha_{3}+3 \cdot \alpha_{4}+2 \cdot \alpha_{5}+1 \cdot \alpha_{6}+0 \cdot \alpha_{7}+0 \cdot \alpha_{8} \\
& =\frac{1}{2}\left(e_{1}+e_{2}+e_{3}+e_{4}+e_{5}-e_{6}-e_{7}+e_{8}\right)
\end{aligned}
$$

and we have ht $\left(\alpha_{69}\right)=11$. In particular, we have

$$
U E_{6}=\prod_{\alpha \in \Phi_{6}^{+}} X_{\alpha}, \quad U E_{7}=\prod_{\alpha \in \Phi_{7}^{+}} X_{\alpha} \text { and } U E_{8}=\prod_{\alpha \in \Phi_{8}^{+}} X_{\alpha} .
$$

Let $i \in\{6,7\}$. By factoring out the unipotent radical $\prod_{\gamma \in \Phi_{8}^{+} \backslash \Phi_{i}^{+}} X_{\gamma}$ we can identify the group $U E_{i}$ with a factor group of $U E_{8}$ in a natural way, and it follows from Remark 2 that for each $\alpha \in \Phi_{i}^{+}$we have $\Phi_{8}^{+} \backslash \Phi_{i}^{+} \subseteq k(\alpha)$.

To formulate the next result we introduce the following set of positive roots:

$$
\begin{aligned}
R_{6 / 7 / 8}^{\text {normal }}:=\Phi_{8}^{+} \backslash & \left\{\alpha_{45}, \alpha_{53}, \alpha_{57}, \alpha_{59}, \alpha_{60}, \alpha_{64}, \alpha_{67}, \alpha_{70}, \alpha_{71}, \alpha_{72}, \alpha_{73}, \alpha_{76}, \alpha_{77},\right. \\
& \alpha_{78}, \alpha_{80}, \alpha_{83}, \alpha_{84}, \alpha_{85}, \alpha_{86}, \alpha_{87}, \alpha_{88}, \alpha_{89}, \alpha_{90}, \alpha_{91}, \alpha_{92}, \alpha_{94}, \alpha_{95}, \alpha_{98}, \\
& \alpha_{99}, \alpha_{100}, \alpha_{102}, \alpha_{103}, \alpha_{104}, \alpha_{105}, \alpha_{106}, \alpha_{107}, \alpha_{108}, \alpha_{109}, \alpha_{110}, \alpha_{111}, \\
& \left.\alpha_{113}, \alpha_{114}, \alpha_{115}, \alpha_{116}, \alpha_{117}, \alpha_{118}\right\} .
\end{aligned}
$$

The next proposition includes a construction of all single root midafis of the groups $U E_{6}, U E_{7}$, $U E_{8}$ and proves Theorems 1.2 and 1.3 for root systems $\Phi$ of type $E_{6}, E_{7}$ and $E_{8}$. The proof of the proposition is based on computer calculations. These calculations are carried out with the help of computer programs which we have implemented in CHEVIE $[\mathbf{1 0}, \mathbf{3 0}]$. 
We proceed as follows. Let $i \in\{6,7,8\}$ and let $\alpha \in \Phi_{i}^{+}$. We choose an arm $a(\alpha)=$ $\left\{\alpha_{i_{1}}, \alpha_{i_{2}}, \ldots, \alpha_{i_{r}}\right\}$ of the hook $h(\alpha)$ such that the indices $i_{1}, i_{2}, \ldots, i_{r}$ are given by the second column of Table A.2. The corresponding leg is $\ell(\alpha)=\{\alpha-\gamma \mid \gamma \in a(\alpha)\}$ and the source is $s(\alpha)=\Phi_{i}^{+} \backslash a(\alpha)$. By computer calculations using CHEVIE we will show that $s(\alpha)$ is a closed pattern. We know from Lemma 3.3 that we can identify the root subgroup $X_{\alpha}$ with the quotient pattern group $S_{\alpha} / P(s(\alpha) \backslash\{\alpha\})$ of the source group $S_{\alpha}=P(s(\alpha))$.

Suppose that $\alpha \in \Phi_{i}^{+} \backslash R_{6 / 7 / 8}^{\text {normal }}$. Let $\bar{\ell}(\alpha):=\ell(\alpha) \cup\left\{a_{j_{1}}, \alpha_{j_{2}}, \ldots, \alpha_{j_{t}}\right\}$ where the indices $j_{1}, j_{2}, \ldots, j_{t}$ are the maxima of the sets of integers given in the third column of Table A.2. By computer calculations using CHEVIE we will show that $\bar{\ell}(\alpha) \cup k(\alpha) \unlhd s(\alpha)$, that the quotient pattern group $P(\bar{\ell}(\alpha) \cup k(\alpha)) / P(k(\alpha))$ is abelian and that $\bar{\ell}(\alpha) \cup\{\alpha\} \cup k(\alpha) \unlhd \Phi_{i}^{+}$. Hence, we obtain the quotient pattern group

$$
\bar{T}_{\alpha}:=S_{\alpha} / P(\{\alpha\} \cup \bar{\ell}(\alpha) \cup k(\alpha)) .
$$

Now suppose that $\alpha \in \Phi_{i}^{+} \cap R_{6 / 7 / 8}^{\text {normal }}$. Again using CHEVIE we will show that $\ell(\alpha) \cup k(\alpha) \unlhd s(\alpha)$ and that $\ell(\alpha) \cup\{\alpha\} \cup k(\alpha) \unlhd \Phi_{i}^{+}$, so that we can consider the quotient pattern group

$$
\bar{T}_{\alpha}:=S_{\alpha} / P(\{\alpha\} \cup \ell(\alpha) \cup k(\alpha))
$$

Proposition 8.1. Let $i \in\{6,7,8\}$ and let $\Phi_{i}$ be a root system of type $E_{i}$ as described above. For each positive root $\alpha \in \Phi_{i}^{+}$the following are true.

(a) If $\alpha \in \Phi_{i}^{+} \backslash R_{6 / 7 / 8}^{\text {normal }}$ then $\Psi_{\alpha}: \operatorname{Irr}{ }^{\operatorname{lin}}\left(\bar{T}_{\alpha}\right) \times \operatorname{Irr}\left(X_{\alpha}\right)^{*} \rightarrow \operatorname{Irr}^{\text {mida }}\left(U E_{i}\right)_{\alpha}$ with

$$
(\mu, \lambda) \mapsto\left(\operatorname{Infl}_{\bar{T}_{\alpha}}^{S_{\alpha}} \mu \cdot \operatorname{Infl}_{X_{\alpha}}^{S_{\alpha}} \lambda\right)^{U E_{i}}
$$

is a one-to-one correspondence.

(b) If $\alpha \in R_{6 / 7 / 8}^{\text {normal }}$ then $\Psi_{\alpha}: \operatorname{Irr}\left(\bar{T}_{\alpha}\right) \times \operatorname{Irr}\left(X_{\alpha}\right)^{*} \rightarrow \operatorname{Irr}\left(U E_{i}\right)_{\alpha}$ with

$$
(\mu, \lambda) \mapsto\left(\operatorname{Infl}_{\bar{T}_{\alpha}}^{S_{\alpha}} \mu \cdot \operatorname{Inff}_{X_{\alpha}}^{S_{\alpha}} \lambda\right)^{U E_{i}}
$$

is a one-to-one correspondence.

The number $\left|\operatorname{Irr}^{\text {mida }}\left(U E_{i}\right)_{\alpha}\right|$ of midafis for $\alpha$ is given in the second and fifth columns of Table 3 and the degree $\chi(1)$ for $\chi \in \operatorname{Irr}^{\operatorname{mida}}\left(U E_{i}\right)_{\alpha}$ is given in the third and sixth columns of Table 3 .

Remark 4. Let $i \in\{6,7,8\}$ and let $\alpha \in \Phi_{i}^{+}$be a positive root. By definition, all midafis $\chi \in \operatorname{Irr}^{\text {mida }}\left(U E_{i}\right)_{\alpha}$ have the same degree; this is the number given in the third and sixth columns of Table 3 . For example, $\left|\operatorname{Irr}^{\text {mida }}\left(U E_{8}\right)_{\alpha_{115}}\right|=q^{8}(q-1)$ and each $\chi \in \operatorname{Irr}^{\text {mida }}\left(U E_{8}\right)_{\alpha_{115}}$ has degree $\chi(1)=q^{23}$.

The roots in $\Phi_{6}^{+}, \Phi_{7}^{+} \backslash \Phi_{6}^{+}, \Phi_{8}^{+} \backslash \Phi_{7}^{+}$, respectively, are separated from each other by horizontal lines in Tables 3 and A.2.

Proof of Proposition 8.1. The proof is carried out by computer programs which we have implemented in CHEVIE. In particular, for all computations with roots we use these CHEVIE programs.

(a) We demonstrate the proof only for $\alpha=\alpha_{115} \in \Phi_{8}^{+} \backslash R_{6 / 7 / 8}^{\text {normal }}$. The proof for the other roots in $\Phi_{i}^{+} \backslash R_{6 / 7 / 8}^{\text {normal }}$ is similar. Let $\alpha=\alpha_{115}$. Using the data in Table A.1 we see that the hook corresponding to $\alpha$ is 
TABLE 3. Numbers and degrees of the midafis for roots $\alpha_{i} \in \Phi_{8}^{+}$.

\begin{tabular}{|c|c|c|c|c|c|}
\hline Root & Number of midafis & Degree & Root & Number of midafis & Degree \\
\hline$\alpha_{1}$ & $q-1$ & 1 & $\alpha_{2}$ & $q-1$ & 1 \\
\hline$\alpha_{3}$ & $q-1$ & 1 & $\alpha_{4}$ & $q-1$ & 1 \\
\hline$\alpha_{5}$ & $q-1$ & 1 & $\alpha_{6}$ & $q-1$ & 1 \\
\hline$\alpha_{9}$ & $q-1$ & $q$ & $\alpha_{10}$ & $q-1$ & $q$ \\
\hline$\alpha_{11}$ & $q-1$ & $q$ & $\alpha_{12}$ & $q-1$ & $q$ \\
\hline$\alpha_{13}$ & $q-1$ & $q$ & $\alpha_{16}$ & $q(q-1)$ & $q^{2}$ \\
\hline$\alpha_{17}$ & $q(q-1)$ & $q^{2}$ & $\alpha_{18}$ & $q(q-1)$ & $q^{2}$ \\
\hline$\alpha_{19}$ & $q(q-1)$ & $q^{2}$ & $\alpha_{20}$ & $q(q-1)$ & $q^{2}$ \\
\hline$\alpha_{23}$ & $q^{2}(q-1)$ & $q^{3}$ & $\alpha_{24}$ & $q^{2}(q-1)$ & $q^{3}$ \\
\hline$\alpha_{25}$ & $q^{4}(q-1)$ & $q^{3}$ & $\alpha_{26}$ & $q^{2}(q-1)$ & $q^{3}$ \\
\hline$\alpha_{27}$ & $q^{2}(q-1)$ & $q^{3}$ & $\alpha_{30}$ & $q^{5}(q-1)$ & $q^{4}$ \\
\hline$\alpha_{31}$ & $q^{3}(q-1)$ & $q^{4}$ & $\alpha_{32}$ & $q^{3}(q-1)$ & $q^{4}$ \\
\hline$\alpha_{33}$ & $q^{5}(q-1)$ & $q^{4}$ & $\alpha_{37}$ & $q^{5}(q-1)$ & $q^{5}$ \\
\hline$\alpha_{38}$ & $q^{6}(q-1)$ & $q^{5}$ & $\alpha_{40}$ & $q^{5}(q-1)$ & $q^{5}$ \\
\hline$\alpha_{44}$ & $q^{4}(q-1)$ & $q^{6}$ & $\alpha_{45}$ & $q^{7}(q-1)$ & $q^{6}$ \\
\hline$\alpha_{48}$ & $q^{4}(q-1)$ & $q^{6}$ & $\alpha_{51}$ & $q^{6}(q-1)$ & $q^{7}$ \\
\hline$\alpha_{52}$ & $q^{6}(q-1)$ & $q^{7}$ & $\alpha_{57}$ & $q^{6}(q-1)$ & $q^{8}$ \\
\hline$\alpha_{63}$ & $q^{6}(q-1)$ & $q^{9}$ & $\alpha_{69}$ & $q^{5}(q-1)$ & $q^{10}$ \\
\hline$\alpha_{7}$ & $q-1$ & 1 & $\alpha_{14}$ & $q-1$ & $q$ \\
\hline$\alpha_{21}$ & $q(q-1)$ & $q^{2}$ & $\alpha_{28}$ & $q^{2}(q-1)$ & $q^{3}$ \\
\hline$\alpha_{34}$ & $q^{3}(q-1)$ & $q^{4}$ & $\alpha_{35}$ & $q^{3}(q-1)$ & $q^{4}$ \\
\hline$\alpha_{39}$ & $q^{4}(q-1)$ & $q^{5}$ & $\alpha_{41}$ & $q^{6}(q-1)$ & $q^{5}$ \\
\hline$\alpha_{46}$ & $q^{7}(q-1)$ & $q^{6}$ & $\alpha_{49}$ & $q^{6}(q-1)$ & $q^{6}$ \\
\hline$\alpha_{53}$ & $q^{8}(q-1)$ & $q^{7}$ & $\alpha_{55}$ & $q^{6}(q-1)$ & $q^{7}$ \\
\hline$\alpha_{58}$ & $q^{7}(q-1)$ & $q^{8}$ & $\alpha_{59}$ & $q^{8}(q-1)$ & $q^{8}$ \\
\hline$\alpha_{61}$ & $q^{5}(q-1)$ & $q^{8}$ & $\alpha_{64}$ & $q^{8}(q-1)$ & $q^{9}$ \\
\hline$\alpha_{66}$ & $q^{7}(q-1)$ & $q^{9}$ & $\alpha_{70}$ & $q^{8}(q-1)$ & $q^{10}$ \\
\hline$\alpha_{71}$ & $q^{7}(q-1)$ & $q^{10}$ & $\alpha_{75}$ & $q^{7}(q-1)$ & $q^{11}$ \\
\hline$\alpha_{76}$ & $q^{8}(q-1)$ & $q^{11}$ & $\alpha_{80}$ & $q^{7}(q-1)$ & $q^{12}$ \\
\hline$\alpha_{82}$ & $q^{7}(q-1)$ & $q^{12}$ & $\alpha_{85}$ & $q^{7}(q-1)$ & $q^{13}$ \\
\hline$\alpha_{89}$ & $q^{7}(q-1)$ & $q^{14}$ & $\alpha_{93}$ & $q^{7}(q-1)$ & $q^{15}$ \\
\hline$\alpha_{97}$ & $q^{6}(q-1)$ & $q^{16}$ & & & \\
\hline$\alpha_{8}$ & $q-1$ & 1 & $\alpha_{15}$ & $q-1$ & $q$ \\
\hline$\alpha_{22}$ & $q(q-1)$ & $q^{2}$ & $\alpha_{29}$ & $q^{2}(q-1)$ & $q^{3}$ \\
\hline$\alpha_{36}$ & $q^{3}(q-1)$ & $q^{4}$ & $\alpha_{42}$ & $q^{4}(q-1)$ & $q^{5}$ \\
\hline$\alpha_{43}$ & $q^{4}(q-1)$ & $q^{5}$ & $\alpha_{47}$ & $q^{5}(q-1)$ & $q^{6}$ \\
\hline$\alpha_{50}$ & $q^{7}(q-1)$ & $q^{6}$ & $\alpha_{54}$ & $q^{8}(q-1)$ & $q^{7}$ \\
\hline$\alpha_{56}$ & $q^{7}(q-1)$ & $q^{7}$ & $\alpha_{60}$ & $q^{9}(q-1)$ & $q^{8}$ \\
\hline$\alpha_{62}$ & $q^{7}(q-1)$ & $q^{8}$ & $\alpha_{65}$ & $q^{8}(q-1)$ & $q^{9}$ \\
\hline$\alpha_{67}$ & $q^{9}(q-1)$ & $q^{9}$ & $\alpha_{68}$ & $q^{7}(q-1)$ & $q^{9}$ \\
\hline$\alpha_{72}$ & $q^{9}(q-1)$ & $q^{10}$ & $\alpha_{73}$ & $q^{9}(q-1)$ & $q^{10}$ \\
\hline$\alpha_{74}$ & $q^{6}(q-1)$ & $q^{10}$ & $\alpha_{77}$ & $q^{9}(q-1)$ & $q^{11}$ \\
\hline$\alpha_{78}$ & $q^{9}(q-1)$ & $q^{11}$ & $\alpha_{79}$ & $q^{8}(q-1)$ & $q^{11}$ \\
\hline$\alpha_{81}$ & $q^{8}(q-1)$ & $q^{12}$ & $\alpha_{83}$ & $q^{10}(q-1)$ & $q^{12}$ \\
\hline
\end{tabular}


Table 3 (Continued).

\begin{tabular}{cccccc}
\hline Root & Number of midafis & Degree & Root & Number of midafis & Degree \\
\hline$\alpha_{84}$ & $q^{8}(q-1)$ & $q^{12}$ & $\alpha_{86}$ & $q^{9}(q-1)$ & $q^{13}$ \\
$\alpha_{87}$ & $q^{9}(q-1)$ & $q^{13}$ & $\alpha_{88}$ & $q^{9}(q-1)$ & $q^{13}$ \\
$\alpha_{90}$ & $q^{9}(q-1)$ & $q^{14}$ & $\alpha_{91}$ & $q^{8}(q-1)$ & $q^{14}$ \\
$\alpha_{92}$ & $q^{9}(q-1)$ & $q^{14}$ & $\alpha_{94}$ & $q^{9}(q-1)$ & $q^{15}$ \\
$\alpha_{95}$ & $q^{9}(q-1)$ & $q^{15}$ & $\alpha_{96}$ & $q^{8}(q-1)$ & $q^{15}$ \\
$\alpha_{98}$ & $q^{9}(q-1)$ & $q^{16}$ & $\alpha_{99}$ & $q^{9}(q-1)$ & $q^{16}$ \\
$\alpha_{100}$ & $q^{8}(q-1)$ & $q^{16}$ & $\alpha_{101}$ & $q^{8}(q-1)$ & $q^{17}$ \\
$\alpha_{102}$ & $q^{9}(q-1)$ & $q^{17}$ & $\alpha_{103}$ & $q^{9}(q-1)$ & $q^{17}$ \\
$\alpha_{104}$ & $q^{8}(q-1)$ & $q^{18}$ & $\alpha_{105}$ & $q^{9}(q-1)$ & $q^{18}$ \\
$\alpha_{106}$ & $q^{8}(q-1)$ & $q^{18}$ & $\alpha_{107}$ & $q^{8}(q-1)$ & $q^{19}$ \\
$\alpha_{108}$ & $q^{9}(q-1)$ & $q^{19}$ & $\alpha_{109}$ & $q^{8}(q-1)$ & $q^{20}$ \\
$\alpha_{110}$ & $q^{9}(q-1)$ & $q^{20}$ & $\alpha_{111}$ & $q^{9}(q-1)$ & $q^{21}$ \\
$\alpha_{112}$ & $q^{8}(q-1)$ & $q^{21}$ & $\alpha_{113}$ & $q^{8}(q-1)$ & $q^{22}$ \\
$\alpha_{114}$ & $q^{8}(q-1)$ & $q^{22}$ & $\alpha_{115}$ & $q^{8}(q-1)$ & $q^{23}$ \\
$\alpha_{116}$ & $q^{8}(q-1)$ & $q^{24}$ & $\alpha_{117}$ & $q^{8}(q-1)$ & $q^{25}$ \\
$\alpha_{118}$ & $q^{8}(q-1)$ & $q^{26}$ & $\alpha_{119}$ & $q^{8}(q-1)$ & $q^{27}$ \\
$\alpha_{120}$ & $q^{7}(q-1)$ & $q^{28}$ & & & \\
\hline
\end{tabular}

$$
\begin{aligned}
& h(\alpha)=\left\{\gamma \in \Phi_{8}^{+} \mid \text {there is } \gamma^{\prime} \in \Phi_{8}^{+} \text {such that } \gamma+\gamma^{\prime}=\alpha_{115}\right\} \\
&=\left\{\alpha_{2}, \alpha_{3}, \alpha_{9}, \alpha_{17}, \alpha_{23}, \alpha_{25}, \alpha_{30}, \alpha_{33}, \alpha_{38}, \alpha_{41}, \alpha_{44}, \alpha_{46}, \alpha_{50}, \alpha_{51}, \alpha_{54},\right. \\
& \alpha_{57}, \alpha_{58}, \alpha_{64}, \alpha_{65}, \alpha_{69}, \alpha_{71}, \alpha_{72}, \alpha_{75}, \alpha_{78}, \alpha_{80}, \alpha_{81}, \alpha_{84}, \alpha_{85}, \alpha_{86}, \alpha_{90}, \\
& \alpha_{91}, \alpha_{93}, \alpha_{95}, \alpha_{97}, \alpha_{98}, \alpha_{100}, \alpha_{101}, \alpha_{102}, \alpha_{104}, \alpha_{105}, \alpha_{107}, \alpha_{108}, \alpha_{109}, \\
&\left.\alpha_{112}, \alpha_{113}, \alpha_{114}, \alpha_{115}\right\} .
\end{aligned}
$$

According to Table A.2 we make the following choice for the arm and the leg of the hook $h(\alpha)$ :

$$
\begin{aligned}
a(\alpha)=\{ & \alpha_{2}, \alpha_{3}, \alpha_{9}, \alpha_{17}, \alpha_{23}, \alpha_{25}, \alpha_{30}, \alpha_{33}, \alpha_{38}, \alpha_{41}, \alpha_{44}, \alpha_{46}, \alpha_{50}, \alpha_{51}, \alpha_{54}, \alpha_{57}, \\
& \left.\alpha_{58}, \alpha_{64}, \alpha_{65}, \alpha_{71}, \alpha_{72}, \alpha_{78}, \alpha_{84}\right\} \\
\ell(\alpha)=\{ & \alpha_{69}, \alpha_{75}, \alpha_{80}, \alpha_{81}, \alpha_{85}, \alpha_{86}, \alpha_{90}, \alpha_{91}, \alpha_{93}, \alpha_{95}, \alpha_{97}, \alpha_{98}, \alpha_{100}, \alpha_{101}, \alpha_{102}, \\
& \left.\alpha_{104}, \alpha_{105}, \alpha_{107}, \alpha_{108}, \alpha_{109}, \alpha_{112}, \alpha_{113}, \alpha_{114}\right\} .
\end{aligned}
$$

Using CHEVIE we verify that $s(\alpha)=\Phi_{8}^{+} \backslash a(\alpha)$ is a closed pattern and using Definition 8 we get $k(\alpha)=\left\{\alpha_{116}, \alpha_{117}, \alpha_{118}, \alpha_{119}, \alpha_{120}\right\}$. In particular, we have $\ell(\alpha) \cup k(\alpha) \subseteq s(\alpha)$. The normal closure of $\ell(\alpha) \cup k(\alpha)$ in $s(\alpha)$ is $\bar{\ell}(\alpha) \cup k(\alpha)$ where

$$
\bar{\ell}(\alpha)=\left\{\alpha_{89}, \alpha_{94}, \alpha_{99}, \alpha_{103}, \alpha_{106}, \alpha_{110}, \alpha_{111}\right\} \cup \ell(\alpha)
$$

and using CHEVIE we verify that $\bar{\ell}(\alpha) \cup\{\alpha\} \cup k(\alpha) \unlhd \Phi_{8}^{+}$. Also by a direct calculation we see that $h(\alpha) \cup k(\alpha)$ is a closed pattern. We set

$$
S_{\alpha}:=P(s(\alpha)) \quad \text { and } \quad H_{\alpha}:=P(h(\alpha) \cup k(\alpha)) / P(k(\alpha)) .
$$

We claim that $H_{\alpha}$ is special of type $q^{1+2|a(\alpha)|}=q^{1+2 \cdot 23}$ and that $\left[x, H_{\alpha}\right]=Z\left(H_{\alpha}\right)=X_{\alpha}$ for all $x \in H_{\alpha} \backslash Z\left(H_{\alpha}\right)$. The proof is analogous to that of Lemma 7.2(d): a direct calculation shows that for all $\gamma, \gamma^{\prime} \in h(\alpha)$ with $\gamma+\gamma^{\prime} \in \Phi_{8}^{+}$we have $\gamma+\gamma^{\prime} \in\{\alpha\} \cup k(\alpha)$. It follows that $X_{\alpha} \subseteq Z\left(H_{\alpha}\right)$ 
and $\left[H_{\alpha}, H_{\alpha}\right] \subseteq X_{\alpha}$. Now let $x \in H_{\alpha} \backslash X_{\alpha}$. We write $x=\prod_{\gamma \in h(\alpha)} x_{\gamma}\left(t_{\gamma}\right)$ as in (2.1). Because $x \notin X_{\alpha}$ there is some $\gamma \in h(\alpha) \backslash\{\alpha\}$ such that $t_{\gamma} \neq 0$. Thus, $\gamma^{\prime}:=\alpha-\gamma \in h(\alpha)$ and we get from Lemma 3.1(a) that $\left\{\left[x, x_{\gamma^{\prime}}(t)\right] \mid t \in \mathbb{F}_{q}\right\}=X_{\alpha}=Z\left(H_{\alpha}\right)=\left[x, H_{\alpha}\right]=\left[H_{\alpha}, H_{\alpha}\right]=\Phi\left(H_{\alpha}\right)$. So $H_{\alpha}$ is special of type $q^{1+2|a(\alpha)|}=q^{1+2 \cdot 23}$. Note that this argument also shows that $a(\alpha) \cup k(\alpha)$ and $\ell(\alpha) \cup k(\alpha)$ are closed patterns and that the quotient pattern groups $P(a(\alpha) \cup k(\alpha)) / P(k(\alpha))$ and $P(\ell(\alpha) \cup k(\alpha)) / P(k(\alpha))$ are abelian groups.

To apply Lemmas 2.1 and 6.1 we introduce the following notation (only for this proof):

- $U:=U E_{8} / P(k(\alpha))$,

- $H:=\bar{S}_{\alpha}:=S_{\alpha} / P(k(\alpha)) \subseteq U$,

- $Z:=X_{\alpha} \subseteq U$,

- $X:=\prod_{\gamma \in a(\alpha)} X_{\gamma} \subseteq U$,

- $Y:=P(\bar{\ell}(\alpha) \cup k(\alpha)) / P(k(\alpha)) \subseteq U$,

- $\operatorname{Irr}(U)_{\alpha}:=\left\{\chi \in \operatorname{Irr}(U) \mid X_{\alpha} \not \subset \operatorname{Ker}(\chi)\right\}$,

where $X_{\gamma}$ denotes the image of $X_{\gamma}$ in $U$.

We have just seen that $X$ is an abelian group and it follows from (2.1) that $X$ is a set of representatives for $U / H$. A direct calculation shows that for all $\gamma, \gamma^{\prime} \in \bar{\ell}(\alpha)$ such that $\gamma+\gamma^{\prime} \in \Phi_{8}^{+}$we have $\gamma+\gamma^{\prime} \in k(\alpha)$. Thus $Y$ is abelian. By Lemma 5.4 and Definition 6(a) we have $Z \subseteq Z(U)$. We have $Y \unlhd H$ and $Z Y \unlhd U$ because $\bar{\ell}(\alpha) \cup k(\alpha) \unlhd s(\alpha)$ and $\bar{\ell}(\alpha) \cup\{\alpha\} \cup$ $k(\alpha) \unlhd \Phi_{8}^{+}$. Furthermore, we have $Y \cap Z=\{1\}$ because $\alpha \notin \bar{\ell}(\alpha)$. Hence, conditions (a)(d) in Lemma 2.1 are satisfied. Suppose that $\lambda \in \operatorname{Irr}(Z)^{*}$ and let $\tilde{\lambda}$ be the inflation of $\lambda$ to

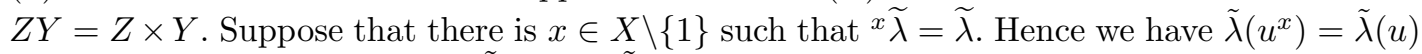
for all $u \in Z Y$. In particular, $\tilde{\lambda}\left(u^{x}\right)=\tilde{\lambda}(u)$ for all $u \in L_{\alpha}:=P(\ell(\alpha) \cup k(\alpha)) / P(k(\alpha))$ and therefore $\tilde{\lambda}([u, x])=1$ for all $u \in L_{\alpha}$. It follows that $[u, x] \in \operatorname{Ker}(\tilde{\lambda})$ for all $u \in L_{\alpha}$ and then also $[x, u]=[u, x]^{-1} \in \operatorname{Ker}(\tilde{\lambda})$ for all $u \in L_{\alpha}$. Since $X$ is abelian and $Z \subseteq Z(U)$ we get that $[x, u] \in \operatorname{Ker}(\tilde{\lambda})$ for all $u \in X Z L_{\alpha}=H_{\alpha}$ and hence $Z=\left[x, H_{\alpha}\right] \subseteq \operatorname{Ker}(\tilde{\lambda})$. Thus $1=\tilde{\lambda}(Z)=\lambda(Z)$ which is impossible since $\lambda$ is non-trivial. Hence, condition (e) of Lemma 2.1 is also satisfied.

It follows from Lemmas 3.3 and 5.1 that $H / Y \cong \bar{T}_{\alpha} \times X_{\alpha}$ where

$$
\bar{T}_{\alpha}:=S_{\alpha} / P(\{\alpha\} \cup \bar{\ell}(\alpha) \cup k(\alpha)),
$$

and Lemma 2.1 gives a one-to-one correspondence

$$
\tilde{\Psi}_{\alpha}: \operatorname{Irr}\left(\bar{T}_{\alpha}\right) \times \operatorname{Irr}\left(X_{\alpha}\right)^{*} \rightarrow \operatorname{Irr}(U)_{\alpha} \cap \operatorname{Irr}\left(U, \mathbf{1}_{Y}\right), \quad(\mu, \lambda) \mapsto\left(\operatorname{Infl}_{\bar{T}_{\alpha}}^{H} \mu \cdot \operatorname{Infl}_{X_{\alpha}}^{H} \lambda\right)^{U}
$$

We are interested in the characters $\chi \in \operatorname{Irr}(U)_{\alpha}$ such that the degree $\chi(1)$ is minimal. Let $d:=\min \left\{\chi(1) \mid \chi \in \operatorname{Irr}(U)_{\alpha}\right\}$ and $\operatorname{Irr}^{\text {mida }}(U)_{\alpha}:=\left\{\chi \in \operatorname{Irr}(U)_{\alpha} \mid \chi(1)=d\right\}$. For every $\lambda \in \operatorname{Irr}\left(X_{\alpha}\right)^{*}$ we have

$$
\tilde{\Psi}_{\alpha}(\mu, \lambda)(1) \begin{cases}=[U: H]=q^{|a(\alpha)|}=q^{23} & \text { if } \mu \text { is linear, } \\ >q^{23} & \text { if } \mu \text { is non-linear. }\end{cases}
$$

Let $\chi \in \operatorname{Irr}(U)_{\alpha} \backslash \operatorname{Irr}\left(U, \mathbf{1}_{Y}\right)$. We claim that $\chi(1)>q^{23}$. There are exactly $q-1$ irreducible characters of $H_{\alpha}$ not having $X_{\alpha}$ in their kernel, namely the characters $\left(\operatorname{Infl}_{X_{\alpha}}^{X_{\alpha} \times L_{\alpha}} \lambda\right)^{H_{\alpha}}$ where $\lambda \in \operatorname{Irr}\left(X_{\alpha}\right)^{*}$. Each such character has degree $q^{|a(\alpha)|}=q^{23}$. Since $X_{\alpha} \not \subset \operatorname{Ker}(\chi)$ it follows that $\chi \in \operatorname{Irr}\left(U, \mathbf{1}_{L_{\alpha}}\right)$. Thus the restriction $\left.\chi\right|_{Z Y}$ has an irreducible constituent $\tilde{\mu} \in \operatorname{Irr}\left(Z Y, \mathbf{1}_{L_{\alpha}}\right)$. Note that $\tilde{\mu}$ is a linear character since $Y$ and $Z Y=Z \times Y$ are abelian. Since $\chi \in \operatorname{Irr}(U)_{\alpha}$, $Z Y \unlhd U$ and $Z \subseteq Z(U)$ we have $X_{\alpha} \not \subset \operatorname{Ker}(\tilde{\mu})$, and since $\chi \notin \operatorname{Irr}\left(U, \mathbf{1}_{Y}\right)$ we have $\left.\tilde{\mu}\right|_{Y} \neq \mathbf{1}_{Y}$. 
It follows that there is some root

$$
\beta \in \bar{\ell}(\alpha) \backslash \ell(\alpha)=\left\{\alpha_{89}, \alpha_{94}, \alpha_{99}, \alpha_{103}, \alpha_{106}, \alpha_{110}, \alpha_{111}\right\}
$$

such that $X_{\beta} \not \subset \operatorname{Ker}(\tilde{\mu})$. We only demonstrate the case $\beta=\alpha_{106}$. The other cases are similar.

We choose the subhook $h^{\prime}(\beta)=\left\{\alpha_{5}, \alpha_{103}, \beta=\alpha_{106}\right\}$ according to Table A.2. A direct calculation shows that $h^{\prime}(\beta) \cup k(\alpha)$ is a closed pattern so that condition (a) of Lemma 6.1 is satisfied. The fact $\alpha_{5}+\alpha_{103}=\beta$ implies that the group $H_{\beta}^{\prime}:=P\left(h^{\prime}(\beta) \cup k(\alpha)\right) / P(k(\alpha))$ satisfies condition (e) of Lemma 6.1. We have already seen above that $H_{\alpha}$ satisfies condition (d) of Lemma 6.1 and also condition (c) is obviously satisfied. As in Lemma 6.1, let $h_{\alpha \beta}^{\prime}$ be the closed pattern generated by $h(\alpha) \cup h^{\prime}(\beta) \cup k(\alpha)$ so that

$$
\begin{aligned}
h_{\alpha \beta}^{\prime}=\{ & \alpha_{2}, \alpha_{3}, \alpha_{5}, \alpha_{9}, \alpha_{17}, \alpha_{23}, \alpha_{25}, \alpha_{30}, \alpha_{33}, \alpha_{38}, \alpha_{41}, \alpha_{44}, \alpha_{46}, \alpha_{50}, \alpha_{51}, \alpha_{54}, \\
& \alpha_{57}, \alpha_{58}, \alpha_{64}, \alpha_{65}, \alpha_{69}, \alpha_{71}, \alpha_{72}, \alpha_{75}, \alpha_{78}, \alpha_{80}, \alpha_{81}, \alpha_{84}, \alpha_{85}, \alpha_{86}, \alpha_{90}, \\
& \alpha_{91}, \alpha_{93}, \alpha_{95}, \alpha_{97}, \alpha_{98}, \alpha_{100}, \alpha_{101}, \alpha_{102}, \alpha_{103}, \alpha_{104}, \alpha_{105}, \alpha_{106}, \alpha_{107}, \\
& \left.\alpha_{108}, \alpha_{109}, \alpha_{112}, \alpha_{113}, \alpha_{114}, \alpha_{115}, \alpha_{116}, \alpha_{117}, \alpha_{118}, \alpha_{119}, \alpha_{120}\right\} .
\end{aligned}
$$

A direct calculation shows that $h_{\alpha \beta}^{\prime} \backslash a(\alpha)$ normalizes $\ell(\alpha) \cup k(\alpha)$ so that condition (b) of Lemma 6.1 is also satisfied. Define $\ell_{\alpha \beta}^{\prime}:=\{\alpha, \beta\} \cup \ell(\alpha) \cup k(\alpha)$ and $L_{\alpha \beta}^{\prime}:=P\left(\ell_{\alpha \beta}^{\prime}\right) / P(k(\alpha))$ as in Lemma 6.1. Let $\mu:=\left.\tilde{\mu}\right|_{L_{\alpha \beta}^{\prime}}$. Since $\tilde{\mu}$ is a linear character we have $\mu \in \operatorname{Irr}\left(L_{\alpha \beta}^{\prime}\right)$ and the properties of $\tilde{\mu}$ imply that $\mu$ satisfies the assumptions in Lemma 6.1. Because $\mu$ is a constituent of $\left.\chi\right|_{L_{\alpha \beta}^{\prime}}$ there is a constituent $\psi \in \operatorname{Irr}\left(H_{\alpha \beta}^{\prime}, \mu\right)$ of $\left.\chi\right|_{H_{\alpha \beta}^{\prime}}$ and Lemma 6.1 gives us $\chi(1) \geqslant \psi(1) \geqslant q^{|a(\alpha)|+1}>q^{23}$. In particular, we get that the degree $\chi(1)$ is not minimal among the degrees of the irreducible characters in $\operatorname{Irr}(U)_{\alpha}$.

Using a similar argument for the other roots $\beta \in \bar{\ell}(\alpha) \backslash \ell(\alpha)$, we get that $\chi(1)>q^{23}$ for all $\chi \in \operatorname{Irr}(U)_{\alpha} \backslash \operatorname{Irr}\left(U, \mathbf{1}_{Y}\right)$. It follows that $d=q^{23}$ and

$$
\operatorname{Irr}^{\text {mida }}(U)_{\alpha}=\left\{\chi \in \operatorname{Irr}(U)_{\alpha} \mid \chi(1)=q^{23}\right\} \subseteq \operatorname{Irr}(U)_{\alpha} \cap \operatorname{Irr}\left(U, \mathbf{1}_{Y}\right) .
$$

Hence $\tilde{\Psi}_{\alpha}$ maps $\operatorname{Irr}^{\operatorname{lin}}\left(\bar{T}_{\alpha}\right) \times \operatorname{Irr}\left(X_{\alpha}\right)^{*}$ one-to-one onto the set of irreducible characters in $\operatorname{Irr}(U)_{\alpha}$ of minimal degree. Identifying the irreducible characters of $U$ with their inflations to $U E_{8}$, we see that the map $\Psi_{\alpha}$ defined in part (a) of the proposition maps $\operatorname{Irr}^{\operatorname{lin}}\left(\bar{T}_{\alpha}\right) \times \operatorname{Irr}\left(\mathbb{F}_{q}\right)^{*}$ one-to-one onto $\operatorname{Irr}^{\text {mida }}\left(U E_{8}\right)_{\alpha}$. This completes the proof of part (a) of the proposition.

(b) We demonstrate the proof only for $\alpha=\alpha_{112} \in \Phi_{8}^{+} \cap R_{6 / 7 / 8}^{\text {normal }}$. The proof for the other roots in $\Phi_{i}^{+} \cap R_{6 / 7 / 8}^{\text {normal }}$ is similar. Let $\alpha=\alpha_{112}$. Using the data in Table A.1 we see that the hook corresponding to $\alpha$ is

$$
\begin{aligned}
& h(\alpha)=\left\{\gamma \in \Phi_{8}^{+} \mid \text {there is } \gamma^{\prime} \in \Phi_{8}^{+} \text {such that } \gamma+\gamma^{\prime}=\alpha_{112}\right\} \\
&=\{ \alpha_{2}, \alpha_{10}, \alpha_{17}, \alpha_{18}, \alpha_{25}, \alpha_{26}, \alpha_{32}, \alpha_{33}, \alpha_{34}, \alpha_{40}, \alpha_{41}, \alpha_{42}, \alpha_{48}, \alpha_{49}, \alpha_{50}, \alpha_{55}, \\
& \alpha_{56}, \alpha_{61}, \alpha_{62}, \alpha_{68}, \alpha_{69}, \alpha_{74}, \alpha_{75}, \alpha_{80}, \alpha_{81}, \alpha_{85}, \alpha_{86}, \alpha_{89}, \alpha_{90}, \alpha_{91}, \alpha_{93}, \alpha_{94}, \\
&\left.\alpha_{95}, \alpha_{98}, \alpha_{99}, \alpha_{100}, \alpha_{102}, \alpha_{103}, \alpha_{105}, \alpha_{106}, \alpha_{108}, \alpha_{110}, \alpha_{112}\right\} .
\end{aligned}
$$

According to Table A.2 we make the following choice for the arm and the leg of the hook $h(\alpha)$ :

$$
\begin{aligned}
a(\alpha)=\{ & \alpha_{2}, \alpha_{10}, \alpha_{17}, \alpha_{18}, \alpha_{25}, \alpha_{26}, \alpha_{32}, \alpha_{33}, \alpha_{34}, \alpha_{40}, \alpha_{41}, \alpha_{42}, \alpha_{48}, \alpha_{49}, \alpha_{50}, \\
& \left.\alpha_{55}, \alpha_{56}, \alpha_{61}, \alpha_{62}, \alpha_{68}, \alpha_{74}\right\}, \\
\ell(\alpha)=\{ & \alpha_{69}, \alpha_{75}, \alpha_{80}, \alpha_{81}, \alpha_{85}, \alpha_{86}, \alpha_{89}, \alpha_{90}, \alpha_{91}, \alpha_{93}, \alpha_{94}, \alpha_{95}, \alpha_{98}, \alpha_{99}, \alpha_{100}, \\
& \left.\alpha_{102}, \alpha_{103}, \alpha_{105}, \alpha_{106}, \alpha_{108}, \alpha_{110}\right\} .
\end{aligned}
$$

Using CHEVIE we verify that $s(\alpha)=\Phi_{8}^{+} \backslash a(\alpha)$ is a closed pattern, and using Definition 8 we get

$$
k(\alpha)=\left\{\alpha_{97}, \alpha_{101}, \alpha_{104}, \alpha_{107}, \alpha_{109}, \alpha_{111}, \alpha_{113}, \alpha_{114}, \alpha_{115}, \alpha_{116}, \alpha_{117}, \alpha_{118}, \alpha_{119}, \alpha_{120}\right\} .
$$


In particular, $\ell(\alpha) \cup k(\alpha) \subseteq s(\alpha)$. Using CHEVIE we verify that $\ell(\alpha) \cup k(\alpha) \unlhd s(\alpha)$, that $\ell(\alpha) \cup\{\alpha\} \cup k(\alpha) \unlhd \Phi_{8}^{+}$and that for all $\gamma, \gamma^{\prime} \in \ell(\alpha)$ with $\gamma+\gamma^{\prime} \in \Phi_{8}^{+}$we have $\gamma+\gamma^{\prime} \in k(\alpha)$. Let $a(\alpha)=\left\{\alpha_{i_{1}}, \alpha_{i_{2}}, \ldots, \alpha_{i_{r}}\right\}$ where $i_{1}<i_{2}<\ldots<i_{r}$. Again using CHEVIE we see that for all $j, k \in\{1,2, \ldots, r\}$ with $k>j$ we have $\alpha_{i_{k}}+\left(\alpha-\alpha_{i_{j}}\right) \notin \Phi_{8}^{+}$or $\alpha_{i_{k}}+\left(\alpha-\alpha_{i_{j}}\right) \in k(\alpha)$. We set $S_{\alpha}:=P(s(\alpha))$. To apply Lemma 2.1 we introduce the following notation (only for this proof):

- $U:=U E_{8} / P(k(\alpha))$,

- $H:=\bar{S}_{\alpha}:=S_{\alpha} / P(k(\alpha)) \subseteq U$,

- $Z:=X_{\alpha} \subseteq U$,

- $X:=\prod_{\gamma \in a(\alpha)} X_{\gamma} \subseteq U$,

- $Y:=P(\ell(\alpha) \cup k(\alpha)) / P(k(\alpha)) \subseteq U$,

- $\operatorname{Irr}(U)_{\alpha}:=\left\{\chi \in \operatorname{Irr}(U) \mid X_{\alpha} \not \subset \operatorname{Ker}(\chi)\right\}$,

where $X_{\gamma}$ denotes the image of $X_{\gamma}$ in $U$.

It follows from (2.1) that $X$ is a set of representatives for $U / H$. By Lemma 5.4 and Definition 6(a) we have $Z \subseteq Z(U)$. We have $Y \unlhd H$ and $Z Y \unlhd U$ because $\ell(\alpha) \cup k(\alpha) \unlhd s(\alpha)$ and $\ell(\alpha) \cup\{\alpha\} \cup k(\alpha) \unlhd \Phi_{8}^{+}$. Furthermore, we have $Y \cap Z=\{1\}$ because $\alpha \notin \ell(\alpha)$. Hence, the conditions (a)-(d) in Lemma 2.1 are satisfied.

Suppose that $\lambda \in \operatorname{Irr}(Z)^{*}$. Let $\tilde{\lambda}$ be the inflation of $\lambda$ to $Z Y=Z \times Y$. Note that $\tilde{\lambda}$ is a linear character. Suppose that there is $x \in X \backslash\{1\}$ such that ${ }^{x} \widetilde{\lambda}=\widetilde{\lambda}$. Because $\lambda$ is non-trivial on $X_{\alpha}$ there is $t \in \mathbb{F}_{q}$ such that $\widetilde{\lambda}\left(x_{\alpha}(t)\right)=\lambda\left(x_{\alpha}(t)\right) \neq 1$. It follows from Lemma 3.1(a) that there exist some root $\gamma \in \ell(\alpha)$ and an element $t^{\prime} \in \mathbb{F}_{q}$ such that $\left[x, x_{\gamma}\left(t^{\prime}\right)^{-1}\right]=x_{\alpha}(t)$. Hence

$$
{ }^{x} \widetilde{\lambda}\left(x_{\gamma}\left(t^{\prime}\right)\right)=\widetilde{\lambda}\left(x^{-1} \cdot x_{\gamma}\left(t^{\prime}\right) \cdot x \cdot x_{\gamma}\left(t^{\prime}\right)^{-1} \cdot x_{\gamma}\left(t^{\prime}\right)\right)=\widetilde{\lambda}\left(x_{\alpha}(t)\right) \widetilde{\lambda}\left(x_{\gamma}\left(t^{\prime}\right)\right) \neq \widetilde{\lambda}\left(x_{\gamma}\left(t^{\prime}\right)\right),
$$

contradicting ${ }^{x} \widetilde{\lambda}=\widetilde{\lambda}$. Hence condition (e) of Lemma 2.1 holds. Condition (f) of Lemma 2.1 is also satisfied because $|X|=q^{|a(\alpha)|}=|Y|$. It follows from Lemmas 3.3 and 5.1 that $H / Y \cong$ $\bar{T}_{\alpha} \times X_{\alpha}$ where

$$
\bar{T}_{\alpha}:=S_{\alpha} / P(\{\alpha\} \cup \bar{\ell}(\alpha) \cup k(\alpha)) .
$$

Now Lemma 2.1 gives the one-to-one correspondence

$$
\tilde{\Psi}_{\alpha}: \operatorname{Irr}\left(\bar{T}_{\alpha}\right) \times \operatorname{Irr}\left(X_{\alpha}\right)^{*} \rightarrow \operatorname{Irr}(U)_{\alpha}, \quad(\mu, \lambda) \mapsto\left(\operatorname{Infl}_{\bar{T}_{\alpha}}^{H} \mu \cdot \operatorname{Infl}_{X_{\alpha}}^{H} \lambda\right)^{U} .
$$

Identifying the irreducible characters of $U$ with their inflations to $U E_{8}$ gives the one-toone correspondence $\Psi_{\alpha}$ defined in part (b) of the proposition. Note that the bijection $\Psi_{\alpha}$ maps $\operatorname{Irr}{ }^{\operatorname{lin}}\left(\bar{T}_{\alpha}\right) \times \operatorname{Irr}\left(X_{\alpha}\right)^{*}$ onto $\operatorname{Irr}^{\text {mida }}\left(U E_{i}\right)_{\alpha}$. This completes the proof of part (b) of the proposition.

Using Lemma 3.3, we can easily compute $\left|\operatorname{Irr}^{\operatorname{lin}}\left(\bar{T}_{\alpha}\right)\right|=\left|\bar{T}_{\alpha} /\left[\bar{T}_{\alpha}, \bar{T}_{\alpha}\right]\right|$ and get

$$
\begin{aligned}
\left|\operatorname{Irr}^{\operatorname{mida}}\left(U E_{8}\right)_{\alpha}\right| & =\left|\Psi_{\alpha}\left(\operatorname{Irr}{ }^{\operatorname{lin}}\left(\bar{T}_{\alpha}\right) \times \operatorname{Irr}\left(X_{\alpha}\right)^{*}\right)\right|=\left|\operatorname{Irr}^{\operatorname{lin}}\left(\bar{T}_{\alpha}\right)\right| \cdot\left|\operatorname{Irr}\left(\mathbb{F}_{q}\right)^{*}\right| \\
& =\left|\operatorname{Irr}^{\operatorname{lin}}\left(\bar{T}_{\alpha}\right)\right| \cdot(q-1) .
\end{aligned}
$$

For every $\chi \in \operatorname{Irr}^{\text {mida }}\left(U E_{8}\right)_{\alpha}$ we have $\chi(1)=\Psi_{\alpha}(\mu, \lambda)(1)$ for some linear character $\mu$ of $\bar{T}_{\alpha}$ and $\lambda \in \operatorname{Irr}\left(X_{\alpha}\right)^{*}$ and hence

$$
\chi(1)=\Psi_{\alpha}(\mu, \lambda)(1)=\left[U E_{8}: S_{\alpha}\right] \cdot \mu(1) \cdot \lambda(1)=q^{|a(\alpha)|} .
$$

This gives the entries in Table 3 and completes the proof of Proposition 8.1. 
REMARK 5. Let $i \in\{6,7,8\}$ and let $\alpha \in \Phi_{i}^{+}$be a positive root.

(a) We could not find a canonical choice for the arm $a(\alpha)$. However, in some sense the choices in Table A.2 are best possible; see Remark 7.

(b) For roots $\alpha \in \Phi_{i}^{+} \cap R_{6 / 7 / 8}^{\text {normal }}$ Proposition 8.1(b) reduces the classification of the midafis in $\operatorname{Irr}\left(U E_{i}\right)_{\alpha}$ to the character theory of the subquotient $\bar{T}_{\alpha}$. We illustrate using the example of $\alpha=\alpha_{112}=\frac{1}{2}\left(e_{1}+e_{2}+e_{3}+e_{4}+e_{5}+e_{6}+e_{7}+e_{8}\right)$ for $i=8$ how information on the structure of $\bar{T}_{\alpha}$ can be obtained. The following are true:

(1) $n(\alpha)=\left\{e_{8}+e_{j} \mid 1 \leqslant j \leqslant 7\right\}=\left\{\alpha_{113}, \alpha_{115}, \alpha_{116}, \alpha_{117}, \alpha_{118}, \alpha_{119}, \alpha_{120}\right\}$,

(2) $k(\alpha)=\left\{e_{8} \pm e_{j} \mid 1 \leqslant j \leqslant 7\right\}=\left\{\alpha_{97}, \alpha_{101}, \alpha_{104}, \alpha_{107}, \alpha_{109}, \alpha_{111}, \alpha_{113}\right.$, $\left.\alpha_{114}, \alpha_{115}, \alpha_{116}, \alpha_{117}, \alpha_{118}, \alpha_{119}, \alpha_{120}\right\}$,

(3) $a(\alpha)=\left\{e_{i}+e_{j} \mid 1 \leqslant j<i \leqslant 7\right\}=\left\{\alpha_{2}, \alpha_{10}, \alpha_{17}, \alpha_{18}, \alpha_{25}, \alpha_{26}, \alpha_{32}, \alpha_{33}\right.$, $\left.\alpha_{34}, \alpha_{40}, \alpha_{41}, \alpha_{42}, \alpha_{48}, \alpha_{49}, \alpha_{50}, \alpha_{55}, \alpha_{56}, \alpha_{61}, \alpha_{62}, \alpha_{68}, \alpha_{74}\right\}$,

(4) $\ell(\alpha)=\left\{\frac{1}{2}\left( \pm e_{1} \pm e_{2} \pm e_{3} \pm e_{4} \pm e_{5} \pm e_{6} \pm e_{7}+e_{8}\right) \mid\right.$ exactly two signs are negative $\}=\left\{\alpha_{69}, \alpha_{75}, \alpha_{80}, \alpha_{81}, \alpha_{85}, \alpha_{86}, \alpha_{89}, \alpha_{90}, \alpha_{91}, \alpha_{93}\right.$, $\left.\alpha_{94}, \alpha_{95}, \alpha_{98}, \alpha_{99}, \alpha_{100}, \alpha_{102}, \alpha_{103}, \alpha_{105}, \alpha_{106}, \alpha_{108}, \alpha_{110}\right\}$.

Equations (3) and (4) are the result of the calculations recorded in Table A.2. The other equations can be confirmed by our CHEVIE programs, but they can also be proved by hand. Equation (1) follows from the equation

$$
\begin{aligned}
e_{8} \pm e_{j}= & \frac{1}{2}\left( \pm e_{1} \pm \ldots \pm e_{j-1} \pm e_{j} \pm e_{j+1} \ldots \pm e_{7}+e_{8}\right) \\
& +\frac{1}{2}\left(\mp e_{1} \mp \ldots \mp e_{j-1} \pm e_{j} \mp e_{j+1} \ldots \mp e_{7}+e_{8}\right) .
\end{aligned}
$$

For (2) we observe that $Z\left(U E_{8} / P(n(\alpha))\right)=X_{\alpha}, X_{e_{8}-e_{1}}$. Factoring out $X_{e_{8}-e_{1}}$ and repeating yields the claim after seven iterations.

We now define the following subsets of $\Phi_{8}^{+}$:

$$
\begin{aligned}
& a_{6}:=\left\{e_{i}-e_{j} \mid 1 \leqslant j<i \leqslant 7\right\}=\left\{\alpha_{3}, \alpha_{4}, \alpha_{5}, \alpha_{6}, \alpha_{7}, \alpha_{8}, \alpha_{11}, \alpha_{12}, \alpha_{13}, \alpha_{14},\right. \\
& \\
&\left.\alpha_{15}, \alpha_{19}, \alpha_{20}, \alpha_{21}, \alpha_{22}, \alpha_{27}, \alpha_{28}, \alpha_{29}, \alpha_{35}, \alpha_{36}, \alpha_{43}\right\}, \\
& v=\left\{\frac{1}{2}\left( \pm e_{1} \pm e_{2} \pm e_{3} \pm e_{4} \pm e_{5} \pm e_{6} \pm e_{7}+e_{8}\right) \mid \text { exactly two }+ \text { signs }\right\} \\
&=\left\{\alpha_{1}, \alpha_{9}, \alpha_{16}, \alpha_{24}, \alpha_{31}, \alpha_{39}, \alpha_{47}\right\} \\
& f:=\left\{\frac{1}{2}\left( \pm e_{1} \pm e_{2} \pm e_{3} \pm e_{4} \pm e_{5} \pm e_{6} \pm e_{7}+e_{8}\right) \mid \text { exactly four }+ \text { signs }\right\} \\
&=\{ \alpha_{23}, \alpha_{30}, \alpha_{37}, \alpha_{38}, \alpha_{44}, \alpha_{45}, \alpha_{46}, \alpha_{51}, \alpha_{52}, \alpha_{53}, \alpha_{54}, \alpha_{57}, \alpha_{58}, \alpha_{59}, \alpha_{60}, \\
& \alpha_{63}, \alpha_{64}, \alpha_{65}, \alpha_{66}, \alpha_{67}, \alpha_{70}, \alpha_{71}, \alpha_{72}, \alpha_{73}, \alpha_{76}, \alpha_{77}, \alpha_{78}, \alpha_{79}, \alpha_{82}, \\
&\left.\alpha_{83}, \alpha_{84}, \alpha_{87}, \alpha_{88}, \alpha_{92}, \alpha_{96}\right\} .
\end{aligned}
$$

With this notation we observe that $s(\alpha)=k(\alpha) \cup\{\alpha\} \cup a_{6} \cup v \cup f \cup \ell(\alpha)$. Also let $K$ denote the standard $D_{7}$-parabolic subgroup of $E_{8}(q)$ with Levi decomposition $K=Q \rtimes D$ (with respect to our choice of the root datum). Then

$$
Z(Q)=P(k(\alpha)), \quad Q / Z(Q)=P(\{\alpha\} \cup v \cup f \cup \ell(\alpha) \cup k(\alpha)) / P(k(\alpha)),
$$

and $\left\langle X_{\gamma} \mid \gamma \in a(\alpha) \cup a_{6} \cup\left(-a_{6}\right)\right\rangle$ is a maximal parabolic subgroup of $D$ with $A_{6}$-Levi factor $A:=\left\langle X_{\gamma} \mid \gamma \in a_{6} \cup\left(-a_{6}\right)\right\rangle$ and unipotent radical $P(a(\alpha))$. We remark that $Z(Q)$ is the natural module and $Q / Z(Q)$ is a half-spin module for $D$. Hence both are elementary abelian. With these facts at our disposal we can now observe the following:

(1) $H_{\alpha}=P(h(\alpha))$ is a special group which is normalized by $P\left(a_{6}\right)$,

(2) $\overline{P(v)}:=P(v \cup k(\alpha)) / P(k(\alpha))$ and $\overline{P(f)}:=P(f \cup k(\alpha)) / P(k(\alpha))$ are elementary abelian and centralize each other, 
(3) $\overline{P(\ell(\alpha))}:=P(\ell(\alpha) \cup k(\alpha)) / P(k(\alpha))$ is normalized by $P\left(a_{6}\right)$ and centralized by $\overline{P(v)} \times \overline{P(f)}$,

(4) $A$ normalizes $\overline{P(v)}$ and $\overline{P(f)}$,

(5) $\overline{P(v)}$ is the natural module for $A$ and $\overline{P(f)}$ is the alternating cube of the natural module,

(6) $\bar{T}_{\alpha}=(\overline{P(v)} \times \overline{P(f)}) \rtimes P\left(a_{6}\right)$,

(7) $P\left(v \cup a_{6}\right) \cong U A_{7}(q)$.

\subsection{Type $F_{4}$}

We construct a root system of type $F_{4}$ as in $[\mathbf{2 3}, \S 12.1]$. Let $e_{1}, e_{2}, e_{3}, e_{4} \in \mathbb{R}^{4}$ be the usual orthonormal unit vectors which form a basis of $\mathbb{R}^{4}$. Then $\Phi_{4}:=\left\{ \pm\left(e_{i} \pm e_{j}\right) \mid 1 \leqslant i \neq j \leqslant\right.$ $4\} \cup\left\{ \pm e_{i} \mid 1 \leqslant i \leqslant 4\right\} \cup\left\{\frac{1}{2}\left( \pm e_{1} \pm e_{2} \pm e_{3} \pm e_{4}\right)\right\}$ is a root system of type $F_{4}$ and the set $\left\{\alpha_{1}, \ldots, \alpha_{4}\right\}$, where $\alpha_{1}:=e_{2}-e_{3}, \alpha_{2}:=e_{3}-e_{4}, \alpha_{3}:=e_{4}$ and $\alpha_{4}:=\frac{1}{2}\left(e_{1}-e_{2}-e_{3}-e_{4}\right)$, is a set of simple roots. The corresponding set of positive roots is

$$
\Phi_{4}^{+}=\left\{e_{i} \pm e_{j} \mid 1 \leqslant i<j \leqslant 4\right\} \cup\left\{e_{i} \mid 1 \leqslant i \leqslant 4\right\} \cup\left\{\frac{1}{2}\left(e_{1} \pm e_{2} \pm e_{3} \pm e_{4}\right)\right\} .
$$

We number the positive roots of $\Phi_{4}$ according to Table A.3. This table contains the following information. The first column fixes the notation for the positive roots of $\Phi_{4}$. The second column lists the coefficients $m_{j}$ when the root $\alpha_{i}=\sum_{j=1}^{4} m_{j} \alpha_{j}$ is written as a linear combination of the simple roots $\alpha_{1}, \alpha_{2}, \alpha_{3}, \alpha_{4}$. The third column expresses the root $\alpha_{i}$ as a linear combination of the vectors $e_{1}, e_{2}, e_{3}, e_{4}$ and the last column contains the height ht $\left(\alpha_{i}\right)$. For example, the positive root $\alpha_{19}$ is

$$
\alpha_{19}=1 \cdot \alpha_{1}+2 \cdot \alpha_{2}+3 \cdot \alpha_{3}+1 \cdot \alpha_{4}=\frac{1}{2}\left(e_{1}+e_{2}+e_{3}+e_{4}\right)
$$

and we have ht $\left(\alpha_{19}\right)=7$.

The construction of the single root midafis of the group $U F_{4}$ is similar to the one for $U E_{i}$. Let $R_{4}^{\text {normal }}:=\Phi_{4}^{+} \backslash\left\{\alpha_{20}, \alpha_{22}\right\}$ and let $\alpha \in \Phi_{4}^{+}$. We choose an arm $a(\alpha)=\left\{\alpha_{i_{1}}, \alpha_{i_{2}}, \ldots, \alpha_{i_{r}}\right\}$ of the hook $h(\alpha)$ such that the indices $i_{1}, i_{2}, \ldots, i_{r}$ are given by the second column of Table A.4. We define and construct the leg $\ell(\alpha)$, the source $s(\alpha)$, the source group $S_{\alpha}$, the enlarged leg $\bar{\ell}(\alpha)$ and the quotient pattern group $\bar{T}_{\alpha}$ of $S_{\alpha}$ in the same way as for $U E_{i}$ (distinguishing the two cases $\alpha \notin R_{4}^{\text {normal }}$ and $\left.\alpha \in R_{4}^{\text {normal }}\right)$.

Proposition 8.2. Let $\Phi_{4}$ be a root system of type $F_{4}$ as described above. For each positive root $\alpha \in \Phi_{4}^{+}$the following are true.

(a) If $\alpha \in \Phi_{4}^{+} \backslash R_{4}^{\text {normal }}$ then $\Psi_{\alpha}: \operatorname{Irr}^{\operatorname{lin}}\left(\bar{T}_{\alpha}\right) \times \operatorname{Irr}\left(X_{\alpha}\right)^{*} \rightarrow \operatorname{Irr}^{\text {mida }}\left(U F_{4}\right)_{\alpha}$ with

$$
(\mu, \lambda) \mapsto\left(\operatorname{Infl}_{\bar{T}_{\alpha}}^{S_{\alpha}} \mu \cdot \operatorname{Infl}_{X_{\alpha}}^{S_{\alpha}} \lambda\right)^{U F_{4}}
$$

is a one-to-one correspondence.

(b) If $\alpha \in R_{4}^{\text {normal }}$ then $\Psi_{\alpha}: \operatorname{Irr}\left(\bar{T}_{\alpha}\right) \times \operatorname{Irr}\left(X_{\alpha}\right)^{*} \rightarrow \operatorname{Irr}\left(U F_{4}\right)_{\alpha}$ with

$$
(\mu, \lambda) \mapsto\left(\operatorname{Infl}_{\bar{T}_{\alpha}}^{S_{\alpha}} \mu \cdot \operatorname{Infl}_{X_{\alpha}}^{S_{\alpha}} \lambda\right)^{U F_{4}}
$$

is a one-to-one correspondence.

The number $\left|\operatorname{Irr}^{\text {mida }}\left(U F_{4}\right)_{\alpha}\right|$ of midafis for $\alpha$ is given in the second and fifth columns of Table 4, and the degree $\chi(1)$ for $\chi \in \operatorname{Irr}^{\text {mida }}\left(U F_{4}\right)_{\alpha}$ is given in the third and sixth columns of Table 4.

Proof. The proof consists of computer calculations carried out by the CHEVIE programs mentioned in $\S 8.1$. The proof is analogous to the proof of Proposition 8.1 with Table A.2 replaced by Table A.4. 


\subsection{Type $G_{2}$}

We construct a root system of type $G_{2}$ as in $[\mathbf{2 3}, \S 12.1]$. Let $e_{1}, e_{2}, e_{3} \in \mathbb{R}^{3}$ be the usual orthonormal unit vectors which form a basis of $\mathbb{R}^{3}$. Then $\Phi_{2}:= \pm\left\{e_{1}-e_{2}, e_{2}-e_{3}, e_{1}-\right.$ $\left.e_{3}, 2 e_{1}-e_{2}-e_{3}, 2 e_{2}-e_{1}-e_{3}, 2 e_{3}-e_{1}-e_{2}\right\}$ is a root system of type $G_{2}$ and the set $\left\{\alpha_{1}, \alpha_{2}\right\}$, where $\alpha_{1}:=e_{1}-e_{2}$ and $\alpha_{2}:=-2 e_{1}+e_{2}+e_{3}$, is a set of simple roots. In particular, $\alpha_{1}$ is a short root and $\alpha_{2}$ is a long root. The corresponding set of positive roots is

$$
\Phi_{2}^{+}=\left\{\alpha_{1}, \alpha_{2}, \alpha_{3}:=\alpha_{1}+\alpha_{2}, \alpha_{4}:=2 \alpha_{1}+\alpha_{2}, \alpha_{5}:=3 \alpha_{1}+\alpha_{2}, \alpha_{6}:=3 \alpha_{1}+2 \alpha_{2}\right\} .
$$

The construction of the single root midafis of the group $U G_{2}$ is similar to the case $\alpha \in R_{i}^{\text {normal }}$ for $U E_{i}$. We define arms of the hooks for the positive roots as follows:

$$
a\left(\alpha_{1}\right):=a\left(\alpha_{2}\right):=\emptyset, \quad a\left(\alpha_{3}\right):=a\left(\alpha_{4}\right):=a\left(\alpha_{5}\right):=\left\{\alpha_{1}\right\}, \quad a\left(\alpha_{6}\right):=\left\{\alpha_{2}, \alpha_{3}\right\},
$$

with corresponding legs $\ell\left(\alpha_{i}\right)$. Let $\alpha \in \Phi_{2}^{+}$. It is easy to see that $s(\alpha):=\Phi_{2}^{+} \backslash a(\alpha)$ is a closed pattern and that $\ell(\alpha) \cup\{\alpha\} \cup k(\alpha) \unlhd \Phi_{2}^{+}$, so that we can consider the quotient pattern group $\bar{T}_{\alpha}:=P(s(\alpha)) / P(\{\alpha\} \cup \ell(\alpha) \cup k(\alpha))$.

Proposition 8.3. Let $\Phi_{2}$ be a root system of type $G_{2}$ as described above. For each positive root $\alpha \in \Phi_{2}^{+}$the map $\Psi_{\alpha}: \operatorname{Irr}\left(\bar{T}_{\alpha}\right) \times \operatorname{Irr}\left(X_{\alpha}\right)^{*} \rightarrow \operatorname{Irr}\left(U G_{2}\right)_{\alpha}$ with

$$
(\mu, \lambda) \mapsto\left(\operatorname{Infl}_{\bar{T}_{\alpha}}^{S_{\alpha}} \mu \cdot \operatorname{Inf}_{X_{\alpha}}^{S_{\alpha}} \lambda\right)^{U E_{i}}
$$

is a one-to-one correspondence. The number $\left|\operatorname{Irr}^{\text {mida }}\left(U G_{2}\right)_{\alpha}\right|$ of midafis for the root $\alpha$ is given in the second and fifth columns of Table 5, and the degree $\chi(1)$ for $\chi \in \operatorname{Irr}^{\text {mida }}\left(U G_{2}\right)_{\alpha}$ is given in the third and sixth columns of Table 5.

TABLE 4. Numbers and degrees of the midafis of $U F_{4}$.

\begin{tabular}{cccccc}
\hline Root & Number of midafis & Degree & Root & Number of midafis & Degree \\
\hline$\alpha_{1}$ & $q-1$ & 1 & $\alpha_{2}$ & $q-1$ & 1 \\
$\alpha_{3}$ & $q-1$ & 1 & $\alpha_{4}$ & $q-1$ & 1 \\
$\alpha_{5}$ & $q-1$ & $q$ & $\alpha_{6}$ & $q-1$ & $q$ \\
$\alpha_{7}$ & $q-1$ & $q$ & $\alpha_{8}$ & $q(q-1)$ & $q^{2}$ \\
$\alpha_{9}$ & $q(q-1)$ & $q$ & $\alpha_{10}$ & $q(q-1)$ & $q^{2}$ \\
$\alpha_{11}$ & $q^{3}(q-1)$ & $q^{2}$ & $\alpha_{12}$ & $q^{2}(q-1)$ & $q^{3}$ \\
$\alpha_{13}$ & $q(q-1)$ & $q^{3}$ & $\alpha_{14}$ & $q^{2}(q-1)$ & $q^{3}$ \\
$\alpha_{15}$ & $q^{5}(q-1)$ & $q^{4}$ & $\alpha_{16}$ & $q^{2}(q-1)$ & $q^{2}$ \\
$\alpha_{17}$ & $q^{4}(q-1)$ & $q^{5}$ & $\alpha_{18}$ & $q^{4}(q-1)$ & $q^{3}$ \\
$\alpha_{19}$ & $q^{3}(q-1)$ & $q^{6}$ & $\alpha_{20}$ & $q^{4}(q-1)$ & $q^{4}$ \\
$\alpha_{21}$ & $q^{3}(q-1)$ & $q^{7}$ & $\alpha_{22}$ & $q^{4}(q-1)$ & $q^{5}$ \\
$\alpha_{23}$ & $q^{4}(q-1)$ & $q^{6}$ & $\alpha_{24}$ & $q^{3}(q-1)$ & $q^{7}$ \\
\hline
\end{tabular}

TABLE 5. Numbers and degrees of the midafis of $U G_{2}$.

\begin{tabular}{cccccc}
\hline Root & Number of midafis & Degree & Root & Number of midafis & Degree \\
\hline$\alpha_{1}$ & $q-1$ & 1 & $\alpha_{2}$ & $q-1$ & 1 \\
$\alpha_{3}$ & $q-1$ & $q$ & $\alpha_{4}$ & $q(q-1)$ & $q$ \\
$\alpha_{5}$ & $q^{2}(q-1)$ & $q$ & $\alpha_{6}$ & $q(q-1)$ & $q^{2}$ \\
\hline
\end{tabular}


Proof. The proof is analogous to the proof of Propositions 8.1 and 8.2 (but the calculations can be carried out by hand).

REMARK 6. It turns out that the group $\bar{T}_{\alpha}$ is elementary abelian for all $\alpha \in \Phi_{2}^{+}$, so that we have $\operatorname{Irr}\left(U G_{2}\right)_{\alpha}=\operatorname{Irr}^{\text {mida }}\left(U G_{2}\right)_{\alpha}$ for all $\alpha \in \Phi_{2}^{+}$. It follows that the only characters $\chi \in \operatorname{Irr}\left(U G_{2}\right)$ which are not single root characters and are not covered by Proposition 8.3 are the trivial character and the linear characters $\chi \in \operatorname{Irr}\left(U G_{2}\right)$ with $|\operatorname{rs}(\chi)|=2$.

Remark 7 . Let $i \in\{2,4,6,7,8\}$ and $\Phi_{i}$ a root system of type $G_{2}, F_{4}, E_{6}, E_{7}, E_{8}$, respectively, with set of positive roots $\Phi_{i}^{+}$as in $\S \S 8.1-8.3$. We use the notation from $\S \S 8.1-8.3$. The arms $a(\alpha)$ in Tables A.2, A.4 and $\S 8.3$ are chosen such that for all $\alpha \in \Phi_{i}^{+}$the following condition is satisfied.

(1) The source $s(\alpha)=\Phi_{i}^{+} \backslash a(\alpha)$ is a closed pattern.

For all roots $\alpha \in \Phi_{i}^{+} \cap R_{i}^{\text {normal }}$ the choice of $a(\alpha)$ in Tables A.2, A.4 and $\S 8.3$ implies that the corresponding leg $\ell(\alpha)$ satisfies the following condition:

(2) $\ell(\alpha) \cup k(\alpha) \unlhd s(\alpha)$.

Now suppose that $\alpha \in \Phi_{i}^{+} \backslash R_{i}^{\text {normal }}$ (where $R_{2}^{\text {normal }}:=\Phi_{2}^{+}$). In this case there is no choice of $a(\alpha)$ such that (1) and (2) are satisfied simultaneously. For each choice of $a(\alpha)$ with corresponding leg $\ell(\alpha)$ such that condition (1) is satisfied let $M$ be the normal closure of $\ell(\alpha) \cup k(\alpha)$ in $s(\alpha)$ and define $\bar{\ell}(\alpha):=M \backslash k(\alpha)$ so that $\bar{\ell}(\alpha) \supsetneq \ell(\alpha)$. For all $\alpha \in \Phi_{i}^{+} \backslash R_{i}^{\text {normal }}$ the choice of $a(\alpha)$ in Tables A.2 and A.4 implies that $\bar{\ell}(\alpha)$ has the following properties:

(3) $\bar{\ell}(\alpha) \cup k(\alpha) \unlhd s(\alpha),\{\alpha\} \cup \bar{\ell}(\alpha) \cup k(\alpha) \unlhd \Phi^{+}$and the quotient pattern group $P(\bar{\ell}(\alpha) \cup$ $k(\alpha)) / P(k(\alpha))$ is abelian.

Among all choices of the arm $a(\alpha)$ such that conditions (1) and (3) hold, the choice in Tables A.2 and A.4 minimizes $|\bar{\ell}(\alpha)|$. This is achieved as follows. Let

$$
\Pi_{\alpha}:=\left\{\left(\alpha_{j}, \alpha_{j^{\prime}}\right) \in \Phi_{i}^{+} \times \Phi_{i}^{+} \mid \alpha_{j}+\alpha_{j^{\prime}}=\alpha \text { and } j<j^{\prime}\right\} .
$$

Among all pairs $\left(\alpha_{j}, \alpha_{j^{\prime}}\right) \in \Pi_{\alpha}$ we choose the (unique) pair where the first index $j$ is maximal (since the roots of each root system are labeled by increasing height, this guarantees that both ht $\left(\alpha_{i}\right)$ and ht $\left(\alpha_{j}\right)$ are 'not too small'). Write $\left(\gamma, \gamma^{\prime}\right)$ for this pair of roots.

For each choice of $a(\alpha)$ we have heart $(\alpha):=\Phi_{i}^{+} \backslash h(\alpha) \subseteq \Phi_{i}^{+} \backslash a(\alpha)=s(\alpha)$. Let $N_{\gamma}$ be the normal closure of $\{\gamma\}$ in the closed pattern generated by heart $(\alpha) \cup\{\gamma\}$, and $N_{\gamma^{\prime}}$ the normal closure of $\left\{\gamma^{\prime}\right\}$ in the closed pattern generated by heart $(\alpha) \cup\left\{\gamma^{\prime}\right\}$. Suppose that the arm $a(\alpha)$ is chosen such that conditions (1) and (2) or conditions (1) and (3) hold. Then we have either $\gamma \in \ell(\alpha)$ or $\gamma^{\prime} \in \ell(\alpha)$. In the first case we have $N_{\gamma} \cap h(\alpha) \subseteq \ell(\alpha)$, and in the second case $N_{\gamma^{\prime}} \cap h(\alpha) \subseteq \ell(\alpha)$. Hence in both cases we have $N_{\gamma} \cap N_{\gamma^{\prime}} \cap h(\alpha) \subseteq \ell(\alpha)$. This considerably reduces the number of possible choices for $\ell(\alpha)$ and hence $a(\alpha)$ such that conditions (1) and (2) or conditions (1) and (3) are satisfied.

Then we do an exhaustive search over the remaining possibilities to filter out those choices which satisfy conditions (1) and (2). If there is such a choice then we know that $\alpha \in R_{i}^{\text {normal }}$ and we choose $a(\alpha)$ such that conditions (1) and (2) hold. If there is no such choice then we know that $\alpha \in \Phi_{i}^{+} \backslash R_{i}^{\text {normal }}$ and we run through all possibilities satisfying conditions (1) and (3) and choose $a(\alpha)$ so that $|\bar{\ell}(\alpha)|$ is minimal.

We consider the example $i=8$ (that is, $\Phi_{i}$ is of type $E_{8}$ ) and $\alpha=\alpha_{115}$. The hook $h(\alpha)$ was determined in the proof of Proposition 8.1. We have $|h(\alpha)|=47$. Hence there are $2^{(|h(\alpha)|-1) / 2}=$ $2^{23}=8388608$ possible choices for the arm $a(\alpha)$. The pair $\left(\alpha_{j}, \alpha_{j^{\prime}}\right) \in \Pi_{\alpha}$ with maximal first index $j$ is $\left(\gamma, \gamma^{\prime}\right)=\left(\alpha_{75}, \alpha_{78}\right)$. Using the CHEVIE programs, we get

$$
\begin{aligned}
& N_{\gamma} \cap N_{\gamma^{\prime}} \cap h(\alpha)=\left\{\alpha_{86}, \alpha_{90}, \alpha_{91}, \alpha_{95}, \alpha_{98}, \alpha_{100}, \alpha_{101}, \alpha_{102}, \alpha_{104}, \alpha_{105}, \alpha_{107},\right. \\
&\left.\alpha_{108}, \alpha_{109}, \alpha_{112}, \alpha_{113}, \alpha_{114}\right\} .
\end{aligned}
$$


It follows that there are at most $2^{23-\left|N_{\gamma} \cap N_{\gamma^{\prime}} \cap h(\alpha)\right|}=2^{23-16}=2^{7}=128$ possible choices such that conditions (1) and (2) or (1) and (3) are satisfied. Testing these 128 possibilities, we see that there is no choice of $a(\alpha)$ such that conditions (1) and (2) are satisfied simultaneously. Thus $\alpha \in \Phi_{8}^{+} \backslash R_{6 / 7 / 8}^{\text {normal }}$. Furthermore, we see that for all choices of $a(\alpha)$ such that conditions (1) and (3) are satisfied simultaneously we have $|\bar{\ell}(\alpha) \backslash \ell(\alpha)| \geqslant 7$. Hence the choice of $a(\alpha)$ in Table A.2 minimizes $|\bar{\ell}(\alpha)|$.

\subsection{Proof of Theorems 1.2 and 1.3}

We can now complete the proof of the main results stated in the introduction.

Proof of Theorem 1.2. Let $\Phi_{i}$ be a root system of type $E_{6}, E_{7}, E_{8}, F_{4}$ or $G_{2}$ as in $\S \S 8.1-8.3$ and $\alpha \in \Phi_{i}^{+}$. If $i \neq 2$ and $\alpha \in \Phi_{i}^{+} \backslash R_{i}^{\text {normal }}$ then the statement of the theorem follows from Propositions 8.1(a) and 8.2(a).

Suppose that $i=2$ or that $\alpha \in R_{i}^{\text {normal }}$. Considering degrees, we see that the one-to-one correspondences $\Psi_{\alpha}$ in Propositions 8.1(b), 8.2(b) and 8.3 map $\operatorname{Irr}^{\operatorname{lin}}\left(\bar{T}_{\alpha}\right) \times \operatorname{Irr}\left(X_{\alpha}\right)^{*}$ onto $\operatorname{Irr}^{\text {mida }}\left(U Y_{i}\right)_{\alpha}$. This completes the proof of Theorem 1.2.

Proof of Theorem 1.3. The theorem follows from Propositions 8.1(b), 8.2(b) and 8.3.

\section{Appendix}

TABle A.1. Positive roots in the root system $\Phi_{8}$ of type $E_{8}$.

\begin{tabular}{|c|c|c|c|c|c|c|c|c|c|c|}
\hline \multirow{2}{*}{$\frac{\text { Root }}{\alpha_{1}}$} & \multicolumn{8}{|c|}{$\begin{array}{l}\text { Linear combination } \\
\text { of simple roots } \\
\alpha_{1} \alpha_{2} \alpha_{3} \alpha_{4} \alpha_{5} \alpha_{6} \alpha_{7} \alpha_{8}\end{array}$} & $\begin{array}{c}\text { Linear combination } \\
\text { of } e_{1}, \ldots, e_{8}\end{array}$ & \multirow{2}{*}{$\frac{\text { Height }}{1}$} \\
\hline & 1 & 0 & ( & ) & ) & 0 & 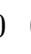 & 0 & $\frac{1}{2}\left(e_{1}-e_{2}-e_{3}-e_{4}-e_{5}-e_{6}-e_{7}+e_{8}\right)$ & \\
\hline$\alpha_{2}$ & 0 & 1 & ) & ) & b & b & 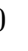 & 0 & $e_{1}+e_{2}$ & 1 \\
\hline$\alpha_{3}$ & 0 & 0 & 1 & ) & ) & b & 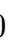 & 0 & $-e_{1}+e_{2}$ & 1 \\
\hline$\alpha_{4}$ & 0 & 0 & 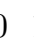 & 1 & ) & b & 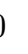 & 0 & $-e_{2}+e_{3}$ & 1 \\
\hline$\alpha_{5}$ & 0 & 0 & ( & ) & 1 & b & 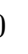 & 0 & $-e_{3}+e_{4}$ & 1 \\
\hline$\alpha_{6}$ & 0 & 0 & 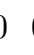 & ) & ) & 1 & ) & 0 & $-e_{4}+e_{5}$ & 1 \\
\hline$\alpha_{7}$ & 0 & 0 & 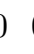 & ) & ) & b & L & 0 & $-e_{5}+e_{6}$ & 1 \\
\hline$\alpha_{8}$ & 0 & 0 & 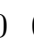 & ) & ) & b & 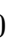 & 1 & $-e_{6}+e_{7}$ & 1 \\
\hline$\alpha_{9}$ & 1 & 0 & 1 & ) & ) & b & ) & 0 & $\frac{1}{2}\left(-e_{1}+e_{2}-e_{3}-e_{4}-e_{5}-e_{6}-e_{7}+e_{8}\right)$ & 2 \\
\hline$\alpha_{10}$ & 0 & 1 & 0 & 1 & 0 & 0 & & 0 & $e_{1}+e_{3}$ & 2 \\
\hline$\alpha_{11}$ & 0 & 0 & 1 & 1 & 0 & 0 & ) & 0 & $-e_{1}+e_{3}$ & 2 \\
\hline$\alpha_{12}$ & 0 & 0 & 0 & 1 & 1 & 0 & & 0 & $-e_{2}+e_{4}$ & 2 \\
\hline$\alpha_{13}$ & 0 & 0 & 0 & ) & 1 & 1 & & 0 & $-e_{3}+e_{5}$ & 2 \\
\hline$\alpha_{14}$ & 0 & 0 & 0 & ) & o & 1 & 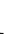 & 0 & $-e_{4}+e_{6}$ & 2 \\
\hline$\alpha_{15}$ & 0 & 0 & 0 & ) & ) & 0 & & 1 & $-e_{5}+e_{7}$ & 2 \\
\hline$\alpha_{16}$ & 1 & 0 & 1 & 1 & 5 & o & & 0 & $\frac{1}{2}\left(-e_{1}-e_{2}+e_{3}-e_{4}-e_{5}-e_{6}-e_{7}+e_{8}\right)$ & 3 \\
\hline$\alpha_{17}$ & 0 & 1 & 1 & 1 & ) & b & & 0 & $e_{2}+e_{3}$ & 3 \\
\hline$\alpha_{18}$ & 0 & 1 & 0 & 1 & 1 & b & & 0 & $e_{1}+e_{4}$ & 3 \\
\hline$\alpha_{19}$ & 0 & 0 & 1 & & 1 & b & & 0 & $-e_{1}+e_{4}$ & 3 \\
\hline$\alpha_{20}$ & 0 & 0 & 0 & 1 & 1 & 1 & & 0 & $-e_{2}+e_{5}$ & 3 \\
\hline
\end{tabular}


Table A.1 (Continued).

\begin{tabular}{|c|c|c|c|c|c|c|c|c|}
\hline \multirow{2}{*}{$\frac{\text { Root }}{\alpha_{21}}$} & \multicolumn{6}{|c|}{$\begin{array}{c}\text { Linear combination } \\
\text { of simple roots } \\
\alpha_{1} \alpha_{2} \alpha_{3} \alpha_{4} \alpha_{5} \alpha_{6} \alpha_{7} \alpha_{8}\end{array}$} & $\begin{array}{l}\text { Linear combination } \\
\text { of } e_{1}, \ldots, e_{8}\end{array}$ & Height \\
\hline & 00 & 00 & 01 & 11 & 11 & 10 & $-e_{3}+e_{6}$ & 3 \\
\hline$\alpha_{22}$ & 00 & 00 & 0 & 0 & 11 & 11 & $-e_{4}+e_{7}$ & 3 \\
\hline$\alpha_{23}$ & 11 & 11 & 1 & 0 & 0 & 00 & $\frac{1}{2}\left(e_{1}+e_{2}+e_{3}-e_{4}-e_{5}-e_{6}-e_{7}+e_{8}\right)$ & 4 \\
\hline$\alpha_{24}$ & 10 & 01 & 11 & 1 & 0 & 00 & $\frac{1}{2}\left(-e_{1}-e_{2}-e_{3}+e_{4}-e_{5}-e_{6}-e_{7}+e_{8}\right)$ & 4 \\
\hline$\alpha_{25}$ & 01 & 11 & 11 & 10 & 0 & 00 & $e_{2}+e_{4}$ & 4 \\
\hline$\alpha_{26}$ & 01 & 10 & 11 & 11 & 1 & 00 & $e_{1}+e_{5}$ & 4 \\
\hline$\alpha_{27}$ & 00 & 01 & 11 & 11 & 1 & 00 & $-e_{1}+e_{5}$ & 4 \\
\hline$\alpha_{28}$ & 00 & 00 & 11 & 11 & 11 & 10 & $-e_{2}+e_{6}$ & 4 \\
\hline$\alpha_{29}$ & 00 & 00 & 01 & 11 & 1 & 11 & $-e_{3}+e_{7}$ & 4 \\
\hline$\alpha_{30}$ & 11 & 11 & 11 & 1 & 0 & 00 & $\frac{1}{2}\left(e_{1}+e_{2}-e_{3}+e_{4}-e_{5}-e_{6}-e_{7}+e_{8}\right)$ & 5 \\
\hline$\alpha_{31}$ & 10 & 01 & 11 & 11 & 1 & 00 & $\frac{1}{2}\left(-e_{1}-e_{2}-e_{3}-e_{4}+e_{5}-e_{6}-e_{7}+e_{8}\right)$ & 5 \\
\hline$\alpha_{32}$ & 01 & 11 & 21 & 1 & 0 & 00 & $e_{3}+e_{4}$ & 5 \\
\hline$\alpha_{33}$ & 01 & 11 & 11 & 11 & 1 & 00 & $e_{2}+e_{5}$ & 5 \\
\hline$\alpha_{34}$ & 01 & 10 & 11 & 11 & 1 & 10 & $e_{1}+e_{6}$ & 5 \\
\hline$\alpha_{35}$ & 00 & 01 & 11 & 11 & 1 & 10 & $-e_{1}+e_{6}$ & 5 \\
\hline$\alpha_{36}$ & 00 & 00 & 11 & 11 & 1 & 11 & $-e_{2}+e_{7}$ & 5 \\
\hline$\alpha_{37}$ & 11 & 11 & 21 & 1 & 0 & 00 & $\frac{1}{2}\left(e_{1}-e_{2}+e_{3}+e_{4}-e_{5}-e_{6}-e_{7}+e_{8}\right)$ & 6 \\
\hline$\alpha_{38}$ & 11 & 11 & 11 & 11 & 1 & 00 & $\frac{1}{2}\left(e_{1}+e_{2}-e_{3}-e_{4}+e_{5}-e_{6}-e_{7}+e_{8}\right)$ & 6 \\
\hline$\alpha_{39}$ & 10 & 01 & 11 & 11 & 1 & 10 & $\frac{1}{2}\left(-e_{1}-e_{2}-e_{3}-e_{4}-e_{5}+e_{6}-e_{7}+e_{8}\right)$ & 6 \\
\hline$\alpha_{40}$ & 01 & 11 & 21 & 11 & 1 & 00 & $e_{3}+e_{5}$ & 6 \\
\hline$\alpha_{41}$ & 01 & 11 & 11 & 11 & 11 & 10 & $e_{2}+e_{6}$ & 6 \\
\hline$\alpha_{42}$ & 01 & 10 & 11 & 11 & 1 & 11 & $e_{1}+e_{7}$ & 6 \\
\hline$\alpha_{43}$ & 00 & 01 & 11 & 11 & 1 & 11 & $-e_{1}+e_{7}$ & 6 \\
\hline$\alpha_{44}$ & 11 & 12 & 21 & 10 & 0 & 00 & $\frac{1}{2}\left(-e_{1}+e_{2}+e_{3}+e_{4}-e_{5}-e_{6}-e_{7}+e_{8}\right)$ & 7 \\
\hline$\alpha_{45}$ & 11 & 11 & 21 & 11 & 1 & 00 & $\frac{1}{2}\left(e_{1}-e_{2}+e_{3}-e_{4}+e_{5}-e_{6}-e_{7}+e_{8}\right)$ & 7 \\
\hline$\alpha_{46}$ & 11 & 11 & 11 & 11 & 1 & 10 & $\frac{1}{2}\left(e_{1}+e_{2}-e_{3}-e_{4}-e_{5}+e_{6}-e_{7}+e_{8}\right)$ & 7 \\
\hline$\alpha_{47}$ & 10 & 01 & 11 & 11 & 1 & 11 & $\frac{1}{2}\left(-e_{1}-e_{2}-e_{3}-e_{4}-e_{5}-e_{6}+e_{7}+e_{8}\right)$ & 7 \\
\hline$\alpha_{48}$ & 01 & 11 & 22 & 21 & 1 & 00 & $e_{4}+e_{5}$ & 7 \\
\hline$\alpha_{49}$ & 01 & 11 & 21 & 11 & 1 & 10 & $e_{3}+e_{6}$ & 7 \\
\hline$\alpha_{50}$ & 01 & 11 & 11 & 11 & 1 & 11 & $e_{2}+e_{7}$ & 7 \\
\hline$\alpha_{51}$ & 11 & 12 & 21 & 11 & 1 & 00 & $\frac{1}{2}\left(-e_{1}+e_{2}+e_{3}-e_{4}+e_{5}-e_{6}-e_{7}+e_{8}\right)$ & 8 \\
\hline$\alpha_{52}$ & 11 & 11 & 2 & 21 & 1 & 00 & $\frac{1}{2}\left(e_{1}-e_{2}-e_{3}+e_{4}+e_{5}-e_{6}-e_{7}+e_{8}\right)$ & 8 \\
\hline$\alpha_{53}$ & 11 & 11 & 21 & 11 & 1 & 10 & $\frac{1}{2}\left(e_{1}-e_{2}+e_{3}-e_{4}-e_{5}+e_{6}-e_{7}+e_{8}\right)$ & 8 \\
\hline$\alpha_{54}$ & 11 & 11 & 11 & 11 & 1 & 11 & $\frac{1}{2}\left(e_{1}+e_{2}-e_{3}-e_{4}-e_{5}-e_{6}+e_{7}+e_{8}\right)$ & 8 \\
\hline$\alpha_{55}$ & 01 & 11 & 2 & 21 & 1 & 10 & $e_{4}+e_{6}$ & 8 \\
\hline$\alpha_{56}$ & 01 & 11 & 21 & 11 & 1 & 11 & $e_{3}+e_{7}$ & 8 \\
\hline$\alpha_{57}$ & 11 & 12 & 2 & 21 & 1 & 00 & $\frac{1}{2}\left(-e_{1}+e_{2}-e_{3}+e_{4}+e_{5}-e_{6}-e_{7}+e_{8}\right)$ & 9 \\
\hline$\alpha_{58}$ & 11 & 12 & 21 & 11 & 1 & 10 & $\frac{1}{2}\left(-e_{1}+e_{2}+e_{3}-e_{4}-e_{5}+e_{6}-e_{7}+e_{8}\right)$ & 9 \\
\hline$\alpha_{59}$ & 11 & 11 & 22 & 21 & 1 & 10 & $\frac{1}{2}\left(e_{1}-e_{2}-e_{3}+e_{4}-e_{5}+e_{6}-e_{7}+e_{8}\right)$ & 9 \\
\hline
\end{tabular}


Table A.1 (Continued).

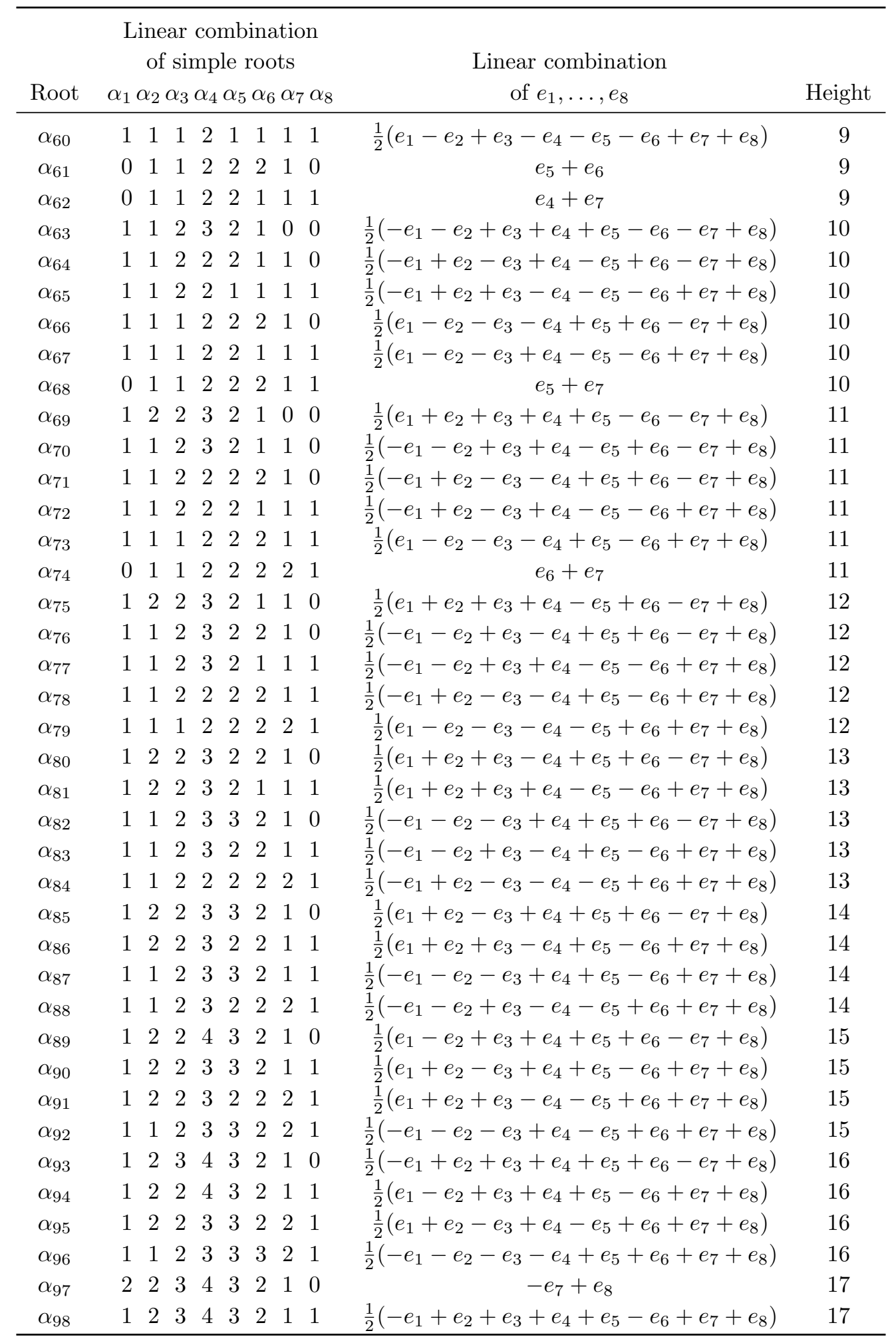


Table A.1 (Continued).

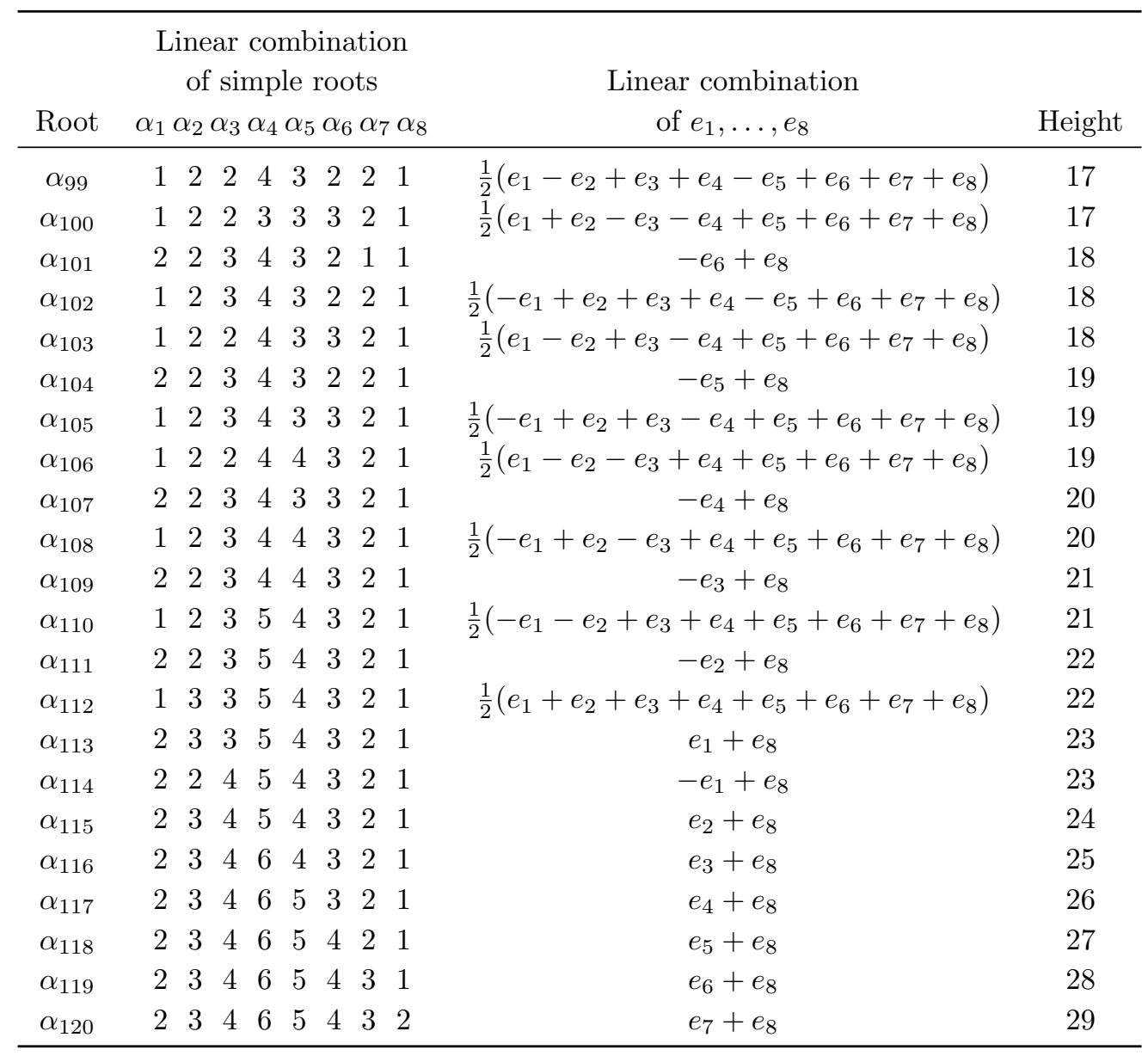


TABle A.2. Arms and subhooks for roots $\alpha \in \Phi_{8}^{+}$.

\begin{tabular}{|c|c|c|}
\hline Root & $\operatorname{Arm} a(\alpha)$ & Subhooks $h^{\prime}(\beta)$ \\
\hline \multicolumn{3}{|l|}{$\alpha_{1}$} \\
\hline \multicolumn{3}{|l|}{$\alpha_{2}$} \\
\hline \multicolumn{3}{|l|}{$\alpha_{3}$} \\
\hline \multicolumn{3}{|l|}{$\alpha_{4}$} \\
\hline \multicolumn{3}{|l|}{$\alpha_{5}$} \\
\hline \multicolumn{3}{|l|}{$\alpha_{6}$} \\
\hline$\alpha_{9}$ & 3 & \\
\hline$\alpha_{10}$ & 4 & \\
\hline$\alpha_{11}$ & 4 & \\
\hline$\alpha_{12}$ & 5 & \\
\hline$\alpha_{13}$ & 6 & \\
\hline$\alpha_{16}$ & 1,4 & \\
\hline$\alpha_{17}$ & 2,3 & \\
\hline$\alpha_{18}$ & 2,5 & \\
\hline$\alpha_{19}$ & 3,5 & \\
\hline$\alpha_{20}$ & 4,6 & \\
\hline$\alpha_{23}$ & $1,2,10$ & \\
\hline$\alpha_{24}$ & $1,5,12$ & \\
\hline$\alpha_{25}$ & $2,3,5$ & \\
\hline$\alpha_{26}$ & $2,6,13$ & \\
\hline$\alpha_{27}$ & $3,6,13$ & \\
\hline$\alpha_{30}$ & $1,2,5,9$ & \\
\hline$\alpha_{31}$ & $1,6,9,13$ & \\
\hline$\alpha_{32}$ & $4,10,11,12$ & \\
\hline$\alpha_{33}$ & $2,3,6,13$ & \\
\hline$\alpha_{37}$ & $1,4,10,12,18$ & \\
\hline$\alpha_{38}$ & $1,2,6,9,13$ & \\
\hline$\alpha_{40}$ & $4,6,10,11,17$ & \\
\hline$\alpha_{44}$ & $3,9,11,16,17,19$ & \\
\hline$\alpha_{45}$ & $1,4,6,10,16,20$ & $\{2,24,30\},\{2,27,33\}$ \\
\hline$\alpha_{48}$ & $5,12,13,18,19,20$ & \\
\hline$\alpha_{51}$ & $3,6,9,11,16,17,23$ & \\
\hline$\alpha_{52}$ & $1,5,12,13,18,20,26$ & \\
\hline$\alpha_{57}$ & $3,5,9,13,19,24,25,30$ & $\{6,32,40\},\{1,40,45\}$ \\
\hline$\alpha_{63}$ & $4,11,12,16,19,20,24,27,31$ & \\
\hline$\alpha_{69}$ & $2,10,17,18,23,25,26,30,32,33$ & \\
\hline$\alpha_{7}$ & & \\
\hline$\alpha_{14}$ & 6 & \\
\hline$\alpha_{21}$ & 5,7 & \\
\hline$\alpha_{28}$ & $4,7,12$ & \\
\hline$\alpha_{34}$ & $2,7,10,14$ & \\
\hline
\end{tabular}


Table A.2 (Continued).

\begin{tabular}{|c|c|c|}
\hline Root & $\operatorname{Arm} a(\alpha)$ & Subhooks $h^{\prime}(\beta)$ \\
\hline$\alpha_{35}$ & $3,7,11,14$ & \\
\hline$\alpha_{39}$ & $1,7,9,14,16$ & \\
\hline$\alpha_{41}$ & $2,3,7,14,21$ & \\
\hline$\alpha_{46}$ & $1,2,7,9,14,21$ & \\
\hline$\alpha_{49}$ & $4,7,10,11,14,17$ & \\
\hline$\alpha_{53}$ & $1,4,7,10,14,16,23$ & $\{11,21,35\},\{2,35,41\}$ \\
\hline$\alpha_{55}$ & $5,7,12,18,19,25,32$ & \\
\hline$\alpha_{58}$ & $3,7,9,11,14,16,17,23$ & \\
\hline$\alpha_{59}$ & $1,5,7,12,18,21,28,34$ & $\begin{array}{l}\{9,20,31\},\{2,31,38\} \\
\{4,38,45\}\end{array}$ \\
\hline$\alpha_{61}$ & $6,13,14,20,21,26,27,28$ & \\
\hline$\alpha_{64}$ & $3,5,7,9,19,21,24,25,30$ & $\begin{array}{l}\{11,34,49\},\{11,38,51\} \\
\{1,49,53\}\end{array}$ \\
\hline$\alpha_{66}$ & $1,6,13,14,20,21,26,28,34$ & \\
\hline$\alpha_{70}$ & $4,7,11,12,16,19,24,32,37,44$ & $\begin{array}{l}\{10,21,34\},\{3,34,41\} \\
\{1,41,46\}\end{array}$ \\
\hline$\alpha_{71}$ & $3,6,9,13,14,21,27,31,33,38$ & $\begin{array}{l}\{7,40,49\},\{1,49,53\} \\
\{5,49,55\},\{1,55,59\}\end{array}$ \\
\hline$\alpha_{75}$ & $2,7,10,17,18,23,25,30,32,37,44$ & \\
\hline$\alpha_{76}$ & $4,6,11,14,16,20,27,28,31,35,39$ & $\begin{array}{l}\{12,33,48\},\{1,48,52\} \\
\{7,48,55\},\{3,52,57\} \\
\{1,55,59\},\{3,59,64\}\end{array}$ \\
\hline$\alpha_{80}$ & $2,6,10,14,17,23,26,33,38,40,45,51$ & $\begin{array}{l}\{7,48,55\},\{1,55,59\} \\
\{3,59,64\},\{4,64,70\}\end{array}$ \\
\hline$\alpha_{82}$ & $5,12,13,19,20,21,24,27,28,31,35,39$ & \\
\hline$\alpha_{85}$ & $2,5,13,18,21,25,26,30,33,34,38,41,46$ & $\begin{array}{l}\{12,51,63\},\{7,63,70\} \\
\{6,70,76\}\end{array}$ \\
\hline$\alpha_{89}$ & $\begin{array}{l}4,10,12,18,20,26,28,32,34,37,40,45 \\
49,53\end{array}$ & $\begin{array}{l}\{5,51,57\},\{5,58,64\} \\
\{6,64,71\}\end{array}$ \\
\hline$\alpha_{93}$ & $\begin{array}{l}3,11,17,19,25,27,32,33,35,40,41,48 \\
49,55,61\end{array}$ & \\
\hline$\alpha_{97}$ & $\begin{array}{l}1,9,16,23,24,30,31,37,38,39,44,45,46 \\
51,52,53\end{array}$ & \\
\hline \multicolumn{3}{|l|}{$\alpha_{8}$} \\
\hline$\alpha_{15}$ & 7 & \\
\hline$\alpha_{22}$ & 6,8 & \\
\hline$\alpha_{29}$ & $5,8,13$ & \\
\hline$\alpha_{36}$ & $4,8,12,15$ & \\
\hline$\alpha_{42}$ & $2,8,10,15,18$ & \\
\hline$\alpha_{43}$ & $3,8,11,15,19$ & \\
\hline$\alpha_{47}$ & $1,8,9,15,16,22$ & \\
\hline$\alpha_{50}$ & $2,3,8,15,22,29$ & \\
\hline
\end{tabular}


Table A.2 (Continued).

\begin{tabular}{|c|c|c|}
\hline Root & $\operatorname{Arm} a(\alpha)$ & Subhooks $h^{\prime}(\beta)$ \\
\hline$\alpha_{54}$ & $1,2,8,9,15,22,29$ & \\
\hline$\alpha_{56}$ & $4,8,10,11,15,17,22$ & \\
\hline$\alpha_{60}$ & $1,4,8,10,15,16,22,23$ & $\{11,29,43\},\{2,43,50\}$ \\
\hline$\alpha_{62}$ & $5,8,12,15,18,19,25,32$ & \\
\hline$\alpha_{65}$ & $3,8,9,11,15,16,17,22,23$ & \\
\hline$\alpha_{67}$ & $1,5,8,12,15,18,24,30,37$ & $\begin{array}{l}\{19,22,43\},\{2,43,50\} \\
\{4,50,56\}\end{array}$ \\
\hline$\alpha_{68}$ & $6,8,13,20,26,27,33,40,48$ & \\
\hline$\alpha_{72}$ & $3,5,8,9,15,19,24,25,29,30$ & $\begin{array}{l}\{11,38,51\},\{11,42,56\}, \\
\{7,51,58\},\{1,56,60\}\end{array}$ \\
\hline$\alpha_{73}$ & $1,6,8,13,20,22,26,29,36,42$ & $\begin{array}{l}\{9,28,39\},\{2,39,46\} \\
\{4,46,53\},\{5,53,59\}\end{array}$ \\
\hline$\alpha_{74}$ & $7,14,15,21,22,28,29,34,35,36$ & \\
\hline$\alpha_{77}$ & $4,8,11,12,15,16,19,24,32,37,44$ & $\{10,29,42\},\{3,42,50\}$ \\
\hline$\alpha_{78}$ & $3,6,8,9,13,22,27,29,31,33,38$ & $\begin{array}{l}\{1,50,54\} \\
\{11,42,56\},\{11,46,58\}, \\
\{1,56,60\},\{5,56,62\}, \\
\{5,58,64\},\{1,62,67\}\end{array}$ \\
\hline$\alpha_{79}$ & $1,7,14,15,21,22,28,29,34,36,42$ & \\
\hline$\alpha_{81}$ & $2,8,10,15,17,18,23,25,30,32,37,44$ & \\
\hline$\alpha_{83}$ & $4,6,8,11,16,20,22,27,31,40,45,51$ & $\begin{array}{l}\{10,29,42\},\{3,42,50\} \\
\{1,50,54\},\{12,50,62\} \\
\{1,62,67\},\{12,58,70\} \\
\{3,67,72\}\end{array}$ \\
\hline$\alpha_{84}$ & $3,7,9,14,15,21,22,29,35,39,41,46$ & $\begin{array}{l}\{8,49,56\},\{1,56,60\} \\
\{5,56,62\},\{1,62,67\} \\
\{6,62,68\},\{1,68,73\}\end{array}$ \\
\hline$\alpha_{86}$ & $2,6,8,10,17,22,23,26,33,38,40,45,51$ & $\begin{array}{l}\{15,48,62\},\{1,62,67\} \\
\{3,67,72\},\{18,58,75\} \\
\{4,72,77\}\end{array}$ \\
\hline$\alpha_{87}$ & $5,8,12,13,19,20,24,27,31,48,52,57,63$ & $\begin{array}{l}\{15,26,42\},\{3,42,50\} \\
\{1,50,54\},\{4,50,56\} \\
\{1,56,60\},\{3,60,65\}\end{array}$ \\
\hline$\alpha_{88}$ & $4,7,11,14,15,16,22,28,35,39,49,53,58$ & $\begin{array}{l}\{8,34,42\},\{3,42,50\}, \\
\{1,50,54\},\{8,55,62\}, \\
\{1,62,67\},\{6,62,68\}, \\
\{3,67,72\},\{1,68,73\}, \\
\{3,73,78\}\end{array}$ \\
\hline$\alpha_{90}$ & $2,5,8,13,18,25,26,29,30,33,38,48,52,57$ & $\begin{array}{l}\{10,43,56\},\{1,56,60\} \\
\{3,60,65\},\{10,64,75\} \\
\{12,65,77\},\{6,75,80\} \\
\{6,77,83\}\end{array}$ \\
\hline
\end{tabular}


Table A.2 (Continued).

\begin{tabular}{|c|c|c|}
\hline Root & $\operatorname{Arm} a(\alpha)$ & Subhooks $h^{\prime}(\beta)$ \\
\hline$\alpha_{91}$ & $2,7,10,14,15,17,22,23,34,41,46,49,53,58$ & $\begin{array}{l}\{8,55,62\},\{1,62,67\} \\
\{6,62,68\},\{3,67,72\} \\
\{1,68,73\},\{4,72,77\} \\
\{3,73,78\},\{4,78,83\}\end{array}$ \\
\hline$\alpha_{92}$ & $\begin{array}{l}5,7,12,15,19,21,24,28,29,35,36,39,43, \\
47\end{array}$ & $\begin{array}{l}\{13,49,61\},\{1,61,66\} \\
\{8,61,68\},\{3,66,71\} \\
\{1,68,73\},\{4,71,76\} \\
\{3,73,78\},\{4,78,83\}\end{array}$ \\
\hline$\alpha_{94}$ & $\begin{array}{l}4,8,10,12,18,20,26,32,37,40,45,48,52,63 \\
69\end{array}$ & $\begin{array}{l}\{11,29,43\},\{1,43,47\}, \\
\{2,43,50\},\{1,50,54\}, \\
\{11,54,65\},\{5,65,72\}, \\
\{6,72,78\}\end{array}$ \\
\hline$\alpha_{95}$ & $\begin{array}{l}2,5,7,15,18,21,25,29,30,34,41,46,55,59, \\
64\end{array}$ & $\begin{array}{l}\{8,49,56\},\{1,56,60\}, \\
\{3,60,65\},\{8,61,68\}, \\
\{1,68,73\},\{8,70,77\}, \\
\{3,73,78\},\{10,71,80\}, \\
\{8,76,83\},\{8,80,86\}, \\
\{8,82,87\},\{14,77,88\}\end{array}$ \\
\hline$\alpha_{96}$ & $\begin{array}{l}6,13,14,20,21,22,27,28,29,31,35,36,39,43 \\
47\end{array}$ & \\
\hline$\alpha_{98}$ & $\begin{array}{l}3,8,11,17,19,25,27,32,33,40,44,48,51,57 \\
63,69\end{array}$ & $\begin{array}{l}\{9,36,47\},\{2,47,54\} \\
\{4,54,60\},\{5,60,67\} \\
\{6,67,73\}\end{array}$ \\
\hline$\alpha_{99}$ & $\begin{array}{l}4,7,10,12,15,18,28,32,34,36,37,42,49,53 \\
55,59\end{array}$ & $\begin{array}{l}\{8,58,65\},\{8,61,68\}, \\
\{5,65,72\},\{1,68,73\}, \\
\{11,66,76\},\{8,71,78\}, \\
\{2,76,80\},\{5,76,82\}, \\
\{8,76,83\},\{14,72,84\}, \\
\{2,82,85\},\{2,83,86\}, \\
\{5,83,87\},\{2,87,90\}\end{array}$ \\
\hline$\alpha_{100}$ & $\begin{array}{l}2,6,13,14,21,22,26,29,33,34,38,41,42,46 \\
50,54\end{array}$ & $\begin{array}{l}\{20,58,76\},\{5,76,82\}, \\
\{8,76,83\},\{5,83,87\}, \\
\{7,83,88\},\{5,88,92\}\end{array}$ \\
\hline$\alpha_{101}$ & $\begin{array}{l}1,8,9,16,23,24,30,31,37,38,44,45,51,52, \\
57,63,69\end{array}$ & \\
\hline$\alpha_{102}$ & $\begin{array}{l}3,7,11,15,17,19,25,32,35,41,43,44,49,50 \\
55,56,62\end{array}$ & $\begin{array}{l}\{9,61,71\},\{4,71,76\}, \\
\{8,71,78\},\{14,67,79\}, \\
\{2,76,80\},\{5,76,82\}, \\
\{4,78,83\},\{2,82,85\}, \\
\{2,83,86\},\{5,83,87\}, \\
\{4,85,89\},\{2,87,90\}, \\
\{4,90,94\}\end{array}$ \\
\hline
\end{tabular}


Table A.2 (Continued).

\begin{tabular}{|c|c|c|}
\hline Root & $\operatorname{Arm} a(\alpha)$ & Subhooks $h^{\prime}(\beta)$ \\
\hline$\alpha_{103}$ & $\begin{array}{l}4,6,10,14,20,22,26,28,34,36,40,42,45,49 \\
53,56,60\end{array}$ & $\begin{array}{l}\{13,58,71\},\{8,71,78\} \\
\{12,71,82\},\{7,78,84\} \\
\{2,82,85\},\{8,82,87\} \\
\{2,87,90\},\{7,87,92\} \\
\{2,92,95\}\end{array}$ \\
\hline$\alpha_{104}$ & $\begin{array}{l}1,7,9,15,16,23,24,30,37,39,44,46,53,58 \\
59,64,70,75\end{array}$ & $\begin{array}{l}\{8,66,73\},\{3,73,78\}, \\
\{4,78,83\},\{2,83,86\} \\
\{5,83,87\},\{2,87,90\} \\
\{4,90,94\},\{3,94,98\}\end{array}$ \\
\hline$\alpha_{105}$ & $\begin{array}{l}3,6,11,14,17,22,27,33,35,40,41,43,49,50 \\
51,56,58,65\end{array}$ & $\begin{array}{l}\{13,53,66\},\{8,66,73\} \\
\{7,73,79\},\{13,70,82\} \\
\{2,82,85\},\{8,82,87\} \\
\{4,85,89\},\{2,87,90\} \\
\{7,87,92\},\{4,90,94\} \\
\{2,92,95\},\{4,95,99\}\end{array}$ \\
\hline$\alpha_{106}$ & $\begin{array}{l}5,12,13,18,20,21,26,28,29,34,36,42,48 \\
52,55,59,61,66\end{array}$ & $\begin{array}{l}\{8,64,72\},\{4,72,77\} \\
\{6,72,78\},\{2,77,81\} \\
\{4,78,83\},\{7,78,84\} \\
\{2,83,86\},\{4,84,88\} \\
\{2,88,91\}\end{array}$ \\
\hline$\alpha_{107}$ & $\begin{array}{l}1,6,9,14,16,22,23,31,38,39,45,46,47,51 \\
53,54,58,60,65\end{array}$ & $\begin{array}{l}\{13,70,82\},\{2,82,85\} \\
\{8,82,87\},\{4,85,89\} \\
\{2,87,90\},\{7,87,92\} \\
\{3,89,93\},\{4,90,94\} \\
\{2,92,95\},\{3,94,98\} \\
\{4,95,99\},\{3,99,102\}\end{array}$ \\
\hline$\alpha_{108}$ & $\begin{array}{l}3,5,13,19,21,25,27,29,33,35,41,43,48,50 \\
55,57,62,64,72\end{array}$ & $\begin{array}{l}\{6,59,66\},\{6,67,73\}, \\
\{6,70,76\},\{7,73,79\}, \\
\{2,76,80\},\{6,77,83\}, \\
\{2,83,86\},\{7,83,88\}, \\
\{12,80,89\},\{2,88,91\}, \\
\{8,89,94\},\{7,94,99\}, \\
\{6,99,103\}\end{array}$ \\
\hline$\alpha_{109}$ & $\begin{array}{l}1,5,9,13,21,24,29,30,31,38,39,46,47,52, \\
54,57,59,64,66,71\end{array}$ & $\begin{array}{l}\{8,70,77\},\{2,77,81\}, \\
\{6,77,83\},\{2,83,86\}, \\
\{7,83,88\},\{12,80,89\}, \\
\{2,88,91\},\{3,89,93\}, \\
\{8,89,94\},\{3,94,98\}, \\
\{7,94,99\},\{3,99,102\}, \\
\{6,99,103\},\{3,103,105\}\end{array}$ \\
\hline
\end{tabular}


Table A.2 (Continued).

\begin{tabular}{|c|c|c|}
\hline Root & $\operatorname{Arm} a(\alpha)$ & Subhooks $h^{\prime}(\beta)$ \\
\hline$\alpha_{110}$ & $\begin{array}{l}4,11,12,19,20,27,28,32,35,36,40,43,48 \\
49,55,56,61,62,68,74\end{array}$ & $\begin{array}{l}\{10,57,69\},\{7,69,75\}, \\
\{6,75,80\},\{8,75,81\}, \\
\{5,80,85\},\{6,81,86\}, \\
\{5,86,90\},\{7,86,91\}, \\
\{5,91,95\},\{6,95,100\}\end{array}$ \\
\hline$\alpha_{111}$ & $\begin{array}{l}1,4,12,16,20,24,28,31,36,37,39,45,47 \\
52,53,59,60,63,66,67,70\end{array}$ & $\begin{array}{l}\{6,72,78\},\{6,75,80\} \\
\{8,75,81\},\{7,78,84\} \\
\{5,80,85\},\{6,81,86\} \\
\{5,86,90\},\{7,86,91\} \\
\{11,85,93\},\{5,91,95\} \\
\{8,93,98\},\{6,95,100\} \\
\{7,98,102\},\{6,102,105\} \\
\{5,105,108\}\end{array}$ \\
\hline$\alpha_{112}$ & $\begin{array}{l}2,10,17,18,25,26,32,33,34,40,41,42,48 \\
49,50,55,56,61,62,68,74\end{array}$ & \\
\hline$\alpha_{113}$ & $\begin{array}{l}1,2,10,18,23,26,30,34,37,38,42,45,46 \\
52,53,54,59,60,66,67,73,79\end{array}$ & $\begin{array}{l}\{17,82,93\},\{8,93,98\} \\
\{7,98,102\},\{6,102,105\} \\
\{5,105,108\},\{4,108,110\}\end{array}$ \\
\hline$\alpha_{114}$ & $\begin{array}{l}3,9,11,16,19,24,27,31,35,39,43,44,47 \\
51,57,58,63,64,70,71,76,82\end{array}$ & $\begin{array}{l}\{8,75,81\},\{6,81,86\} \\
\{5,86,90\},\{7,86,91\} \\
\{4,90,94\},\{5,91,95\} \\
\{4,95,99\},\{6,95,100\} \\
\{4,100,103\},\{5,103,106\}\end{array}$ \\
\hline$\alpha_{115}$ & $\begin{array}{l}2,3,9,17,23,25,30,33,38,41,44,46,50,51 \\
54,57,58,64,65,71,72,78,84\end{array}$ & $\begin{array}{l}\{10,82,89\},\{8,89,94\}, \\
\{7,94,99\},\{6,99,103\}, \\
\{5,103,106\},\{11,106,110\}, \\
\{1,110,111\}\end{array}$ \\
\hline$\alpha_{116}$ & $\begin{array}{l}4,10,11,16,17,23,32,37,40,44,45,49,51 \\
53,56,58,60,63,65,70,76,77,83,88\end{array}$ & $\begin{array}{l}\{2,82,85\},\{2,87,90\}, \\
\{2,92,95\},\{2,96,100\}, \\
\{12,100,106\},\{3,106,108\}, \\
\{1,108,109\}\end{array}$ \\
\hline$\alpha_{117}$ & $\begin{array}{l}5,12,18,19,24,25,30,32,37,44,48,52,55 \\
57,59,62,63,64,67,69,70,72,75,77,81\end{array}$ & $\begin{array}{l}\{13,88,96\},\{2,96,100\}, \\
\{4,100,103\},\{3,103,105\}, \\
\{1,105,107\}\end{array}$ \\
\hline$\alpha_{118}$ & $\begin{array}{l}6,13,20,26,27,31,33,38,40,45,48,51,52 \\
57,61,63,66,68,69,71,73,76,78,82,83,87\end{array}$ & $\begin{array}{l}\{2,88,91\},\{2,92,95\} \\
\{4,95,99\},\{3,99,102\} \\
\{1,102,104\}\end{array}$ \\
\hline$\alpha_{119}$ & $\begin{array}{l}7,14,21,28,34,35,39,41,46,49,53,55,58, \\
59,61,64,66,70,71,75,76,80,82,85,89,93, \\
97\end{array}$ & \\
\hline$\alpha_{120}$ & $\begin{array}{l}8,15,22,29,36,42,43,47,50,54,56,60,62, \\
65,67,68,72,73,74,77,78,79,81,83,84,86, \\
87,88\end{array}$ & \\
\hline
\end{tabular}


TABle A.3. Positive roots in the root system $\Phi_{4}$ of type $F_{4}$.

\begin{tabular}{|c|c|c|c|}
\hline Root & $\begin{array}{c}\text { Linear combination } \\
\text { of simple roots } \\
\alpha_{1} \alpha_{2} \alpha_{3} \alpha_{4}\end{array}$ & $\begin{array}{l}\text { Linear combination } \\
\text { of } e_{1}, e_{2}, e_{3}, e_{4}\end{array}$ & Height \\
\hline$\alpha_{1}$ & $1 \quad 0 \quad 0 \quad 0$ & $e_{2}-e_{3}$ & 1 \\
\hline$\alpha_{2}$ & $\begin{array}{llll}0 & 1 & 0 & 0\end{array}$ & $e_{3}-e_{4}$ & 1 \\
\hline$\alpha_{3}$ & $\begin{array}{lllll}0 & 0 & 1 & 0\end{array}$ & $e_{4}$ & 1 \\
\hline$\alpha_{4}$ & $\begin{array}{llll}0 & 0 & 0 & 1\end{array}$ & $\frac{1}{2}\left(e_{1}-e_{2}-e_{3}-e_{4}\right)$ & 1 \\
\hline$\alpha_{5}$ & $\begin{array}{llll}1 & 1 & 0 & 0\end{array}$ & $e_{2}-e_{4}$ & 2 \\
\hline$\alpha_{6}$ & $\begin{array}{llll}0 & 1 & 1 & 0\end{array}$ & $e_{3}$ & 2 \\
\hline$\alpha_{7}$ & $\begin{array}{lllll}0 & 0 & 1 & 1\end{array}$ & $\frac{1}{2}\left(e_{1}-e_{2}-e_{3}+e_{4}\right)$ & 2 \\
\hline$\alpha_{8}$ & $\begin{array}{llll}1 & 1 & 1 & 0\end{array}$ & $e_{2}$ & 3 \\
\hline$\alpha_{9}$ & $\begin{array}{llll}0 & 1 & 2 & 0\end{array}$ & $e_{3}+e_{4}$ & 3 \\
\hline$\alpha_{10}$ & $\begin{array}{llll}0 & 1 & 1 & 1\end{array}$ & $\frac{1}{2}\left(e_{1}-e_{2}+e_{3}-e_{4}\right)$ & 3 \\
\hline$\alpha_{11}$ & $\begin{array}{llll}1 & 1 & 2 & 0\end{array}$ & $e_{2}+e_{4}$ & 4 \\
\hline$\alpha_{12}$ & $\begin{array}{llll}1 & 1 & 1 & 1\end{array}$ & $\frac{1}{2}\left(e_{1}+e_{2}-e_{3}-e_{4}\right)$ & 4 \\
\hline$\alpha_{13}$ & $\begin{array}{llll}0 & 1 & 2 & 1\end{array}$ & $\frac{1}{2}\left(e_{1}-e_{2}+e_{3}+e_{4}\right)$ & 4 \\
\hline$\alpha_{14}$ & $\begin{array}{llll}1 & 2 & 2 & 0\end{array}$ & $e_{2}+e_{3}$ & 5 \\
\hline$\alpha_{15}$ & $\begin{array}{llll}1 & 1 & 2 & 1\end{array}$ & $\frac{1}{2}\left(e_{1}+e_{2}-e_{3}+e_{4}\right)$ & 5 \\
\hline$\alpha_{16}$ & $\begin{array}{llll}0 & 1 & 2 & 2\end{array}$ & $e_{1}-e_{2}$ & 5 \\
\hline$\alpha_{17}$ & $\begin{array}{llll}1 & 2 & 2 & 1\end{array}$ & $\frac{1}{2}\left(e_{1}+e_{2}+e_{3}-e_{4}\right)$ & 6 \\
\hline$\alpha_{18}$ & $\begin{array}{llll}1 & 1 & 2 & 2\end{array}$ & $e_{1}-e_{3}$ & 6 \\
\hline$\alpha_{19}$ & $\begin{array}{llll}1 & 2 & 3 & 1\end{array}$ & $\frac{1}{2}\left(e_{1}+e_{2}+e_{3}+e_{4}\right)$ & 7 \\
\hline$\alpha_{20}$ & $\begin{array}{llll}1 & 2 & 2 & 2\end{array}$ & $e_{1}-e_{4}$ & 7 \\
\hline$\alpha_{21}$ & $\begin{array}{llll}1 & 2 & 3 & 2\end{array}$ & $e_{1}$ & 8 \\
\hline$\alpha_{22}$ & $\begin{array}{llll}1 & 2 & 4 & 2\end{array}$ & $e_{1}+e_{4}$ & 9 \\
\hline$\alpha_{23}$ & $\begin{array}{llll}1 & 3 & 4 & 2\end{array}$ & $e_{1}+e_{3}$ & 10 \\
\hline$\alpha_{24}$ & $\begin{array}{llll}2 & 3 & 4 & 2\end{array}$ & $e_{1}+e_{2}$ & 11 \\
\hline
\end{tabular}

TABLE A.4. Arms and subhooks for roots $\alpha \in \Phi_{4}^{+}$.

\begin{tabular}{cll}
\hline Root & $\operatorname{Arm} a(\alpha)$ & Subhooks $h^{\prime}(\beta)$ \\
\hline$\alpha_{1}$ & & \\
$\alpha_{2}$ & & \\
$\alpha_{3}$ & & \\
$\alpha_{4}$ & & \\
$\alpha_{5}$ & 2 & \\
$\alpha_{6}$ & 3 & \\
$\alpha_{7}$ & 4 & \\
$\alpha_{8}$ & 1,3 & \\
$\alpha_{9}$ & 3 & \\
$\alpha_{10}$ & 2,4 & \\
$\alpha_{11}$ & 1,3 \\
$\alpha_{12}$ & $1,4,7$ & \\
\hline
\end{tabular}


Table A.4 (Continued).

\begin{tabular}{lll}
\hline Root & $\operatorname{Arm} a(\alpha)$ & Subhooks $h^{\prime}(\beta)$ \\
\hline & & \\
$\alpha_{13}$ & $3,4,7$ & \\
$\alpha_{14}$ & $2,5,6$ & \\
$\alpha_{15}$ & $1,3,4,7$ & \\
$\alpha_{16}$ & 4,7 & \\
$\alpha_{17}$ & $2,4,5,6,8$ & \\
$\alpha_{18}$ & $1,4,7$ & \\
$\alpha_{19}$ & $3,6,7,8,9,10$ & $\{1,13,15\}$ \\
$\alpha_{20}$ & $2,4,5,10$ & \\
$\alpha_{21}$ & $3,4,6,7,8,10,13$ & $\{6,12,17\},\{4,17,20\}$ \\
$\alpha_{22}$ & $3,7,9,11,13$ & \\
$\alpha_{23}$ & $2,6,9,10,13,16$ & \\
$\alpha_{24}$ & $1,5,8,11,12,14,15$ & \\
\hline
\end{tabular}

Acknowledgement. We thank the referee for a careful reading of the manuscript and for saving the authors from incorrect attributions concerning the numbers $N(\Phi)$.

\section{References}

1. C. André, 'Basic characters of the unitriangular group (for arbitrary primes)', Proc. Amer. Math. Soc. 130 (2002) 1943-1954.

2. C. André and A. M. Neto, 'A supercharacter theory for the Sylow p-subgroups of the finite symplectic and orthogonal groups', J. Algebra 322 (2009) 1273-1294.

3. A. Borel and J. Tits, 'Groupes réductifs', Publ. Math. Inst. Hautes Études Sci. 27 (1965) 55-150.

4. R. W. Carter, Simple groups of Lie type, Pure and Applied Mathematics 28 (John Wiley \& Sons, London, 1972).

5. P. Cellini and P. PAPI, 'Ad-nilpotent ideals of a Borel subalgebra. II', J. Algebra 258 (2002) 112-121, special issue in celebration of Claudio Procesi's 60th birthday.

6. P. Diaconis and I. M. IsAacs, 'Supercharacters and superclasses for algebra groups', Trans. Amer. Math. Soc. 360 (2008) 2359-2392.

7. F. Digne and J. Michel, Representations of finite groups of Lie type, London Mathematical Society Student Texts 21 (Cambridge University Press, Cambridge, 1991).

8. D. Ž. Duoković, 'On conjugacy classes of elements of finite order in complex semisimple Lie groups', J. Pure Appl. Algebra 35 (1985) 1-13.

9. S. Fomin and N. REAding, 'Root systems and generalized associahedra, Lecture notes for the IAS/Park City Graduate Summer School in Geometric Combinatorics', Preprint, 2008, arXiv:0505518v3.

10. M. Geck, G. Hiss, F. Lübeck, G. Malle and G. Pfeiffer, 'CHEVIE-a system for computing and processing generic character tables', Appl. Algebra Engrg. Comm. Comput. 7 (1996) 175-210.

11. S. M. Goodwin, T. Le and K. MagaArd, 'The generic character table of a Sylow p-subgroup of a finite Chevalley group of type $D_{4}$ ', Preprint, 2015, arXiv:1508.06937.

12. S. M. Goodwin, T. Le, K. MagaArd and A. Paolini, 'Constructing characters of Sylow p-subgroups of finite Chevalley groups', Preprint, 2015, arXiv:1512.02678.

13. S. M. Goodwin, P. Mosch and G. Röhrle, 'Calculating conjugacy classes in Sylow $p$-subgroups of finite Chevalley groups of rank six and seven', LMS J. Comput. Math. 17 (2014) 109-122.

14. S. M. Goodwin, P. Mosch and G. Röhrle, 'On the coadjoint orbits of maximal unipotent subgroups of reductive groups', Transform. Groups 21 (2016) 399-426.

15. S. M. Goodwin and G. Röhrle, 'Calculating conjugacy classes in Sylow p-subgroups of finite Chevalley groups', J. Algebra 321 (2009) 3321-3334.

16. Z. Halasi, 'On representations of solvable linear groups', $\mathrm{PhD}$ Thesis, Central European University, Budapest, 2009

17. Z. HALASI and P. P. PÁLFY, 'The number of conjugacy classes in pattern groups is not a polynomial function', J. Group Theory 14 (2011) 841-854.

18. G. Higman, 'Enumerating p-groups. I. Inequalities', Proc. Lond. Math. Soc. (3) 10 (1960) 24-30. 
19. F. Himstedt, 'On the decomposition numbers of the Ree groups ${ }^{2} F_{4}\left(q^{2}\right)$ in non-defining characteristic', J. Algebra 325 (2011) 364-403.

20. F. Himstedt and S.-C. HuAng, 'On the decomposition numbers of Steinberg's triality groups ${ }^{3} D_{4}\left(2^{n}\right)$ in odd characteristics', Comm. Algebra 41 (2013) 1484-1498.

21. F. Himstedt, T. Le and K. Magandd, 'Characters of the Sylow p-subgroups of the Chevalley groups $D_{4}\left(p^{n}\right)^{\prime}$, J. Algebra 332 (2011) 414-427.

22. F. Himstedt and F. Noeske, 'Decomposition numbers of $\mathrm{SO}_{7}(q)$ and $\mathrm{Sp}_{6}(q)$ ', J. Algebra 413 (2014) 15-40.

23. J. E. Humphreys, Introduction to Lie algebras and representation theory, Graduate Texts in Mathematics 9 (Springer, New York, 1997).

24. B. Huppert, Endliche Gruppen I, Die Grundlehren der Mathematischen Wissenschaften 134 (Springer, Berlin, 1967).

25. I. M. IsAaCS, Character theory of finite groups (Dover Publications, New York, 1994).

26. I. M. IsAACS, 'Counting characters of upper triangular groups', J. Algebra 315 (2007) 698-719.

27. T. LE, 'Irreducible characters of the unitriangular groups', PhD Thesis, Wayne State University, 2008.

28. T. LE and K. MAgaArd, 'On the character degrees of Sylow $p$-subgroups of Chevalley groups $G\left(p^{f}\right)$ of type E', Forum Math. 27 (2015) 1-55.

29. E. Marberg, 'Heisenberg characters, unitriangular groups, and Fibonacci numbers', J. Combin. Theory Ser. A 119 (2012) 882-903.

30. J. Michel, 'The development version of the CHEVIE package of GAP3', Preprint, 2013, arXiv:1310.7905v2.

31. T. OKUYAma and K. WAKI, 'Decomposition numbers of $\mathrm{Sp}(4, q)$ ', J. Algebra 199 (1998) 544-555.

32. I. PAK and A. Soffer, 'On Higman's $k\left(U_{n}\left(\mathbb{F}_{q}\right)\right)$ conjecture', Preprint, 2015, arXiv:1507.00411.

33. D. I. PANyushev, 'Ad-nilpotent ideals of a Borel subalgebra: generators and duality', J. Algebra 274 (2004) 822-846.

34. J.-Y. SHI, 'The number of $\oplus$-sign types', Quart. J. Math. Oxford Ser. (2) 48 (1997) 93-105.

35. E. N. Sommers, ' $B$-stable ideals in the nilradical of a Borel subalgebra', Canad. Math. Bull. 48 (2005) 460-472.

36. A. Vera-López and J. M. Arregi, 'Conjugacy classes in unitriangular matrices', Linear Algebra Appl. 370 (2003) 85-124.

37. K. WAKI, 'A note on decomposition numbers of $G_{2}\left(2^{n}\right)^{\prime}$, J. Algebra 274 (2004) 602-606.

Frank Himstedt

Technische Universität München

Zentrum Mathematik - M11

Boltzmannstr. 3

85748 Garching

Germany

himstedt@ma.tum.de

Kay Magaard

School of Mathematics

University of Birmingham

Edgbaston

Birmingham B15 2TT

United Kingdom

k.magaard@bham.ac.uk
Tung Le

North-West University

Mmabatho 2735

South Africa

lttung96@yahoo.com 